\title{
The role of oxytocin, testosterone and cortisol \\ in affiliation and bonding in male Barbary macaqaues
}

\author{
Dissertation \\ for the award of the degree \\ "Doctor of Philosophy" (Ph.D.) \\ Division of Mathematics and Natural Sciences \\ at the Georg-August-Universität Göttingen
}

within the doctoral program Behavior and Cognition (BeCog)

of the Georg-August-Universität School of Science (GAUSS)

\author{
Submitted by \\ Alan V. Rincon \\ from Los Angeles, United States of America
}

Göttingen 2019 



\section{Thesis Committee}

Prof. Dr. Julia Ostner

Behavioral Ecology, Georg-August-Universität Göttingen \& Primate Social Evolution, German Primate Center, Leibniz Institute for Primate Research, Göttingen

PD. Dr. Oliver Schülke

Behavioral Ecology, Georg-August-Universität Göttingen \& Primate Social Evolution, German Primate Center, Leibniz Institute for Primate Research, Göttingen

Prof. Dr. Lars Penke

Biological Personality Psychology, Georg-August-Universität Göttingen

\section{Members of the Examination Board}

Reviewer:

Prof. Dr. Julia Ostner

Second Reviewer:

Prof. Dr. Lars Penke

\section{Further members of the Examination Board}

PD. Dr. Oliver Schülke

Behavioral Ecology, Georg-August-Universität Göttingen \& Primate Social Evolution, German Primate Center, Leibniz Institute for Primate Research, Göttingen

Prof. Dr. Julia Fischer

Cognitive Ethology, German Primate Center, Leibniz Institute for Primate Research, Göttingen

Prof. Dr. Eckhart W. Heymann

Behavioural Ecology and Sociobiology Unit, Primate Social Evolution, German Primate Center, Leibniz Institute for Primate Research, Göttingen

Dr. Tanya Behne

Developmental Psychology, Georg-August-Universität Göttingen

\section{Date of oral examination: 21 October 2019}





\section{Contents}

$\begin{array}{ll}\text { Summary } & \text { iii }\end{array}$

1 General Introduction $\quad 1$

1.1 Adaptive benefits of social bonds . . . . . . . . . . . . . . . . . 2

1.2 The steroid/peptide theory of social bonds (S/P theory) . . . . . . . . . . . . 2

1.3 Considerations for non-invasive hormone measurements . . . . . . . . . . . . . . 8

1.4 Study site and species . . . . . . . . . . . . . . . . . . . . . 10

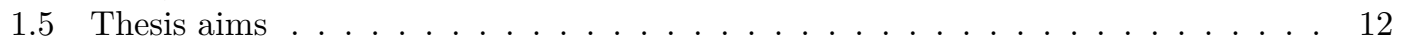

2 Measuring urinary cortisol and testosterone levels in male Barbary macaques: A comparison of EIA and LC-MS 13

Abstract . . . . . . . . . . . . . . . . . . . . . . 14

2.1 Introduction . . . . . . . . . . . . . . . . . . . . . . . . . 15

2.2 Materials and Methods . . . . . . . . . . . . . . . . . . . . . 18

2.3 Results................................. 22

2.4 Discussion ............................ . . . 26

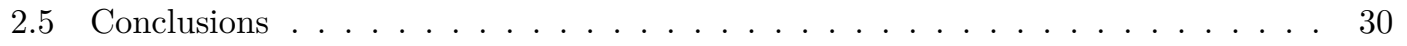

3 Oxytocin increases after affiliative interactions in male Barbary macaques 33

Abstract . . . . . . . . . . . . . . ........... 34

3.1 Introduction . . . . . . . . . . . . . . . . . . . . . 35

3.2 Materials and methods . . . . . . . . . . . . . . . . . . . . . . 37

3.3 Results............................... . . . 42

3.4 Discussion ............................. . . . . . 44

4 Testosterone and cortisol are negatively associated with ritualized bonding behavior in male Barbary macaques 49

Abstract ............................. 50

4.1 Introduction . . . . . . . . . . . . . . . . . . . 51

4.2 Materials and methods . . . . . . . . . . . . . . . . . . . . 53

4.3 Results............................... 56

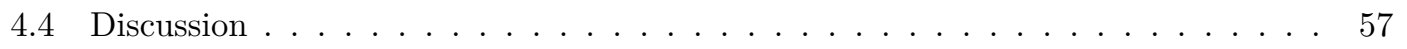

5 General Discussion $\quad 65$

5.1 Summary of results . . . . . . . . . . . . . . . . . . . . . . . 65

5.2 Application of non-invasive methods in behavioral ecology . . . . . . . . . . . . 66

5.3 Oxytocin and social bonding in non-reproductive contexts . . . . . . . . . . . . 67

5.4 Oxytocin, testosterone, cortisol, and same-sex social bonds . . . . . . . . . . 70

5.5 Interaction between HPG and HPA axes in competitive contexts . . . . . . . . . 72

5.6 Conclusions and outlook . . . . . . . . . . . . . . . . . . . 73

$\begin{array}{lr}\text { References } & 77\end{array}$

$\begin{array}{lr}\text { Acknowledgements } & 105\end{array}$ 



\section{Summary}

Many group living mammals minimize the costs of sociality by forming stable affiliative relationships, termed social bonds, with other group members. These bonds are highly adaptive as they increase fitness, and strongly bonded partners often provide each other with social support. However, the dynamics of hormones and behavior regulating social bonds between adults in non-reproductive contexts under natural conditions remain elusive. Three major neuroendocrine systems regulate animal behavior - the oxytocinergic system, the hypothalamic-pituitary-gonadal (HPG) axis, and the hypothalamic-pituitary-adrenal (HPA) axis. Oxytocin is the primary hormone implicated in bond formation in mammals, while the HPG axis is involved in regulating reproductive behaviors, and the HPA axis regulates metabolism and helps individuals cope with social challenges. Each system is able to interact with the other in ways that can fine-tune animal behavior and bonding. The steroid peptide theory of social bonds ( $\mathrm{S} / \mathrm{P}$ theory) proposes that testosterone mediates a trade-off between competition and nurturing/bonding. Thus, the $\mathrm{S} / \mathrm{P}$ theory predicts that the ideal physiological conditions to induce bond formation is high oxytocin coupled with low testosterone levels. Additionally, the HPA axis is highly responsive to the state of an individual's social bonds and support network. Investigating the interplay between physiology and bond formation under natural conditions is an important element of linking sociality to fitness. While methodologies for measuring hormone concentration non-invasively opens up many opportunities to study this interplay under natural conditions, these methods require validation to ensure that their measurements are biologically meaningful.

The overarching aim of this thesis was to use the S/P theory as a framework to study the relationship between oxytocin and testosterone in relation to social bonding between adults and nurturing male infant care under in natural conditions in male Barbary macaques (Macaca sylvanus). As previous studies have suggested that oxytocin release after bonding behaviors is potentially partner specific, I tested for specificity of oxytocin release after two bonding behaviors - grooming and male-infant-male interactions. Furthermore, I predicted low testosterone to occur during male bonding and nurturing infant care as the $\mathrm{S} / \mathrm{P}$ theory predicts that testosterone mediates a trade-off between competition and nurturing. Simultaneously, I predicted that cortisol levels would be negatively correlated with male-infant-male interactions, a behavior that strengthens male bonds and is predictive of coalitionary support. Over the course of my studies I collected over 4000 hours of behavioral data and 650 urine samples from 14 adult males Barbary macaques from a semi-free ranging population. Within this dataset, urine samples were time-matched to social interactions of interest. These events resulted in 152 urine samples for oxytocin and 650 urine samples for testosterone and cortisol each. I additionally collected 32 urine samples from eight immature males for hormone measurement validation purposes. 
I conducted a validation of commonly used testosterone and cortisol enzyme immunoassays (EIA) to assess gonadal and adrenocortical activity from the urine of male Barbary macaques. Measurements from EIA were compared to those measured by liquid chromatography-mass spectrometry (LC-MS) to determine whether EIA measurements accurately reflected the concentration of the target hormones in urine samples. I found that cortisol EIA measurements from unprocessed urine had a strong positive correlation with measurements from LC-MS in both adult and immature males. This strong correlation suggests that the cortisol EIA antibody does not substantially cross-react with other metabolites and is suitable to assess adrenocortical activity in male Barbary macaques of both age classes. EIA measurements of testosterone had a strong positive correlation with LC-MS, but only if steroids in the urine were deconjugated via an enzymatic hydrolysis prior to measurement by EIA in adult males. By contrast, testosterone EIA and LC-MS measurements of samples from immature males did not correlate significantly. As expected, testosterone in adult males was significantly higher than those of immature males when measured by LC-MS but not by EIA, biologically validating testosterone measurements of LC-MS but not EIA. Collectively, these results suggest that the testosterone EIAs are suitable to assess gonadal activity in adult but not immature males, and only if an enzymatic hydrolysis of steroids is performed prior to analysis.

I investigated oxytocin in relation to two bonding behaviors, specifically grooming and male-infant-male interactions. Oxytocin was higher after grooming with any partner. I could not formally test whether oxytocin release was partner specific after grooming as most grooming occurred between bond partners. Nevertheless, this finding is in line with the S/P theory as well as previous studies that found elevated oxytocin following non-sexual affiliative behaviors in other species. Naturally elevated oxytocin levels following grooming interactions did not increase the probability to accept a solicitation for help (from any partner) during an agonistic conflict. It may be that oxytocin does not universally increase the propensity to cooperate, although I cannot exclude the possibility that oxytocin may promote cooperation with bonded partners in within-group conflicts. Following male-infant-male interactions, oxytocin was only elevated after interactions with non-bond partners but not after interactions with bond partners. Furthermore, male-infant-male interactions were negatively associated with testosterone and cortisol. These results support the $\mathrm{S} / \mathrm{P}$ theory proposing that low testosterone is beneficial for bonding. Collectively, these results suggest that male-infant-male interactions serve to create new bonds while not physiologically affecting existing ones. Lastly, testosterone and cortisol levels were both positively correlated with male infant care, suggesting that this behavior is conducted under competitive rather than nurturing contexts. As males preferentially interact with infants based on their past mating success with the mother, infant care could take a protective role, as with most other Old World primates. Alternatively, infant care could be considered mating effort as it increases future copulations with the mother.

Over the course of these studies, I have touched upon the core aspects that define the study of behavioral endocrinology. First, I corroborated previous calls for the need to validate steroid EIAs for each new species or sample matrix, something that needs to be remembered now that these methods are more common. Then, collectively, I was the first to investigate the joint 
levels of oxytocin, testosterone, and cortisol in relation to bonding behavior between same-sex adults under natural conditions. Overall, my thesis provides general support for the S/P theory and demonstrates the benefit of investigating several major hormonal axes regulating animal social behavior, particularly with regards to social bonding. Further studies involving multiple theoretical frameworks and hormonal axes will help elucidate the dynamics of bond formation and maintenance between adults. 



\section{Chapter 1}

\section{General Introduction}

Platonic social bonds, or friendships, between adults are well known to increase fitness in group living mammals (Seyfarth \& Cheney, 2012). Despite extensive research on the benefits of social bonds, however, there are still gaps in our knowledge on how these bonds are formed and maintained. Neuroendocrine mechanisms that evolved in mammals to promote bonds between mother and offspring are conserved across species (Goodson, 2013). These mechanisms have likely been co-opted and adapted to also promote other types of bonds, including those between adults (Numan \& Young, 2016; Ziegler \& Crockford, 2017), where comparatively little is known and less research has been conducted.

Different neuroendocrine systems can act in concert to produce the ideal physiological conditions and behaviors to promote the formation and maintenance of social bonds (van Anders et al., 2011). Oxytocin is the primary neuropeptide hormone implicated in the formation of attachment and partner preferences - key ingredients of a social bond (Ziegler \& Crockford, 2017, 2017). In addition to oxytocin, steroids such as testosterone and cortisol, are powerful mediators of social behavior (Adkins-Regan, 2005) and may interact with oxytocin to either inhibit or facilitate affiliative social interactions and bonding, as implied by functional links between oxytocin and steroid hormones (Neumann, 2002; van Anders et al., 2011). To date, few studies have examined the interplay between oxytocin, testosterone, or cortisol in relation to bond formation under natural social conditions that reflect the evolutionary context under which they have evolved. Developments of techniques for measuring hormones from non-invasively collected samples, such as urine and feces, have made research on the interplay between hormones, behavior, and social bonding in natural conditions increasingly feasible (Behringer \& Deschner, 2017; Palme, 2019). These methodological advancements open a variety of opportunities for investigating the role of endocrinology for bond formation and maintenance in wildlife, a crucial element of understanding social evolution and the links between sociality and fitness.

In the following sections I will begin by outlining the adaptive benefits of platonic social bonds between adults. I will then highlight the three major neuroendocrine systems regulating animal behavior and social relationships - the oxytocinergic system, the hypothalamic-pituitary-gonadal (HPG) axis, and the hypothalamic-pituitary-adrenal (HPA) axis. Next, I introduce the steroid/peptide theory of social bonds ( $\mathrm{S} / \mathrm{P}$ theory), a theoretical framework central to the aims of this thesis. I further discuss each system individually, explaining how they influence animal behavior, social relationships, and ultimately bond formation, while highlighting gaps in the literature. Finally, I will discuss practical considerations when measuring hormones from non-invasively collected samples. 


\subsection{Adaptive benefits of social bonds}

Group living evolves when it provides improved fitness benefits to all group members in comparison to a more solitary lifestyle (Krause \& Ruxton, 2002). Consequently, we expect natural selection to equip individuals within these groups with behavioral strategies to maximize the benefits of group living while minimizing the costs. Many species of mammals ranging from elephants, cetaceans, carnivores and primates, form groups or communities with stable membership where individuals repeatedly interact with each other. When individuals are able to recognize each other, remember past social interactions, and interact repeatedly over time, they develop a social relationship (Hinde, 1976). Several types of social relationships exist and can be broadly classified as either cooperative or competitive. Relationships are differentiated across group members and can be predictive of future interactions (Hinde, 1976). Social bonds are social relationships that are primarily affiliative in nature, stable, equitable, and are often characterized by cooperation and social support (Dunbar \& Shultz, 2010; Massen et al., 2010; Ostner \& Schülke, 2018; Silk, 2002).

Social bonds occur between both kin and non-kin, are found in the philopatric and dispersing sex, and have been shown to increase fitness (reviewed in Seyfarth \& Cheney, 2012). For example, social bonds increase longevity (Archie et al., 2014; Holt-Lunstad et al., 2010; Silk et al., 2010; Stanton \& Mann, 2012), offspring survival (Cameron et al., 2009; Frère et al., 2010; Silk et al., 2009), and reproductive success (Cameron et al., 2009; Schülke et al., 2010). The formation of social bonds might have evolved through selection for the inclusive fitness benefits they provide. As social bonds are often mutualistic in nature, individuals derive direct fitness benefits from forming them (Clutton-Brock, 2009; Silk, 2007). When formed between kin, social bonds also lead to indirect fitness benefits (Clutton-Brock, 2009; Silk, 2007). One potential mechanism linking social bonds to fitness may be its role in promoting cooperation and social support. Strongly bonded partners in several species are more likely to support one another in agonistic conflicts (Cheney, 2011). For example, in male Assamese macaques (Macaca assamensis) strongly bonded males form coalitions more often, which consequently improve dominance rank, and reproductive success (Schülke et al., 2010). Finally, social bonds also allow individuals to better cope with the stressors of life (Cheney \& Seyfarth, 2009; Hostinar et al., 2014; Wittig et al., 2016; Young et al., 2014a). While there is lots of research on benefits of non-reproductive bonds between adults, the neuroendocrine mechanisms regulating them remain understudied.

\subsection{The steroid/peptide theory of social bonds (S/P theory)}

Neuropeptides, sex steroids, and glucocorticoids (GC) are the three major classes of hormones influencing social behavior in vertebrates (Adkins-Regan, 2005; Soares et al., 
2010). Neuropeptides, particularly oxytocin and the oxytocinergic system, are known to affect cognitive processes and behaviors related to nurturance and the formation of social bonds (Numan \& Young, 2016; Ross \& Young, 2009; Ziegler \& Crockford, 2017). The sex steroids, primarily testosterone for males, are regulated by the hypothalamic-pituitary-gonadal (HPG) axis and are often implicated in reproductive behavior (Adkins-Regan, 2005). GCs are regulated by the hypothalamic-pituitary-adrenal (HPA) axis, and regulate metabolism and the response to perceived threats and challenges by mobilizing energy resources (Adkins-Regan, 2005; Sapolsky, 2002). Each of these regulatory systems are closely linked and interact in ways that allow animals to react flexibly to their social environment and respond in an adaptive manner (Neumann, 2002; Salvador, 2012; van Anders et al., 2011). Studying a combination of these systems in conjunction allows us to paint a more complete picture of the endocrine mechanisms underlying social bonding.

The steroid/peptide theory of social bonds (S/P theory) proposes that neuropeptides such as oxytocin form part of a nurturing system that evolved to promote infant survival (van Anders et al., 2011). This nurturing system may promote infant survival by helping parents bond to offspring and/or develop pair bonds (van Anders et al., 2011). The sexual system operates in parallel and involves testosterone, which is proposed to mediate trade-offs between competition for resource acquisition (e.g. status and sexual opportunities) and nurturance (van Anders, 2013; van Anders et al., 2011). Accordingly, the S/P Theory predicts that the ideal physiological conditions to promote nurturance and social bonds are high oxytocin levels coupled with low testosterone levels (van Anders et al., 2011). While not explicitly part of the S/P theory, GCs and the HPA axis have a reciprocal relationship with animal behavior and social support that is also relevant to the study of social bonds (Hostinar et al., 2014).

\subsubsection{The role of oxytocin in social bonding and cooperation}

The formation of social bonds is a process that involves many steps, beginning with the motivation to approach and interact with other individuals, moving into the mental processing of social stimuli and memory of social events, and finally the formation of attachment (Lim \& Young, 2006). As we shall see, it appears that the highly conserved neuropeptide oxytocin plays an important role in many, if not all, of these steps (Lim \& Young, 2006; Ross \& Young, 2009).

The bond between a mother and her offspring is likely the evolutionarily oldest mammalian bond as newborn infants are dependent on their mother for nutrition and survival. Oxytocin released during parturition (Gimpl \& Fahrenholz, 2001) is part of a cascade of physiological changes within the mother that prepares her for the events of giving birth and raising her offspring through dependency. Oxytocin promotes uterine contractions during labor, controls the milk ejection reflex (Gimpl \& Fahrenholz, 2001; Rilling \& Young, 2014), and promotes nurturing maternal behaviors (Numan \& Young, 2016; Ross \& Young, 2009). Beyond simply influencing maternal behavior, oxytocin also creates a partner specific attachment between mother and 
offspring. For example, in sheep, oxytocin released centrally during parturition (Kendrick et al., 1986) facilitates the formation of an olfactory memory of the lamb's odor (Lévy et al., 1995). Once established, this olfactory memory causes the mother to reject nursing attempts lambs that are not her own (Nowak et al., 2011). The ability of oxytocin to create such a preference has likely been co-opted for other types of bonds, including those between adults (Numan \& Young, 2016; Ziegler \& Crockford, 2017).

Oxytocin's involvement in adult bonds has been best studied in the context of pair bonds (Numan \& Young, 2016; Ross \& Young, 2009), a long-lasting sexual relationship involving intimacy between a male and a female. For example, oxytocin released during mating facilitates the formation of a social preference for the mating partner in monogamous prairie vole (Microtus ochrogaster: Ross \& Young, 2009). Oxytocin plays a similar role in pair bonding in monogamous nonhuman primate species (French et al., 2018). For example, oxytocin is positively related to sexual and affiliative behaviors within mate pairs in cotton-top tamarins (Saguinus oedipus: Snowdon et al., 2010), and oxytocin administration strengthens partner preferences in marmosets (Callithrix penicillata: Smith et al., 2010).

The exact mechanism by which oxytocin creates a partner preference in mother-offspring and pair bonds remains unknown. Most likely this partner preference is an outcome of oxytocin's interaction with the reward system, particularly with the dopaminergic pathway (Dölen et al., 2013; Numan \& Young, 2016; Skuse \& Gallagher, 2009; Young \& Wang, 2004). Mating-induced release of oxytocin and dopamine and subsequent activation of the nucleus accumbens (by oxytocin and dopamine) appears to be crucial for the development of a pair bond in monogamous voles (Numan \& Young, 2016; Young \& Wang, 2004). Similar mechanisms are likely at play for other social bonds as the preference to affiliate with a particular partner is a key feature of these relationships.

For oxytocin to regulate bonds in non-reproductive contexts there must be behavioral or contextual triggers that release oxytocin, similar to how parturition or copulations release oxytocin and affect mother-offspring and pair bonds, respectively (Numan \& Young, 2016; Young \& Wang, 2004). Close affiliative contact or socio-positive behaviors may be the behavioral triggers for the release of oxytocin. Indeed, urinary oxytocin levels are positively related to affiliative behaviors between adults in non-sexual contexts in primates (tufted capuchins, Sapajus apella: Benítez et al., 2018; chimpanzees, Pan troglodytes: Crockford et al., 2013; Wittig et al., 2014) and domestic dogs (Canis familiaris: Romero et al., 2014).

Oxytocin release following non-sexual affiliative interactions may depend on the existing relationship quality. For example, urinary oxytocin levels are higher after grooming with bond partners but not after grooming with non-bond partners in chimpanzees (Crockford et al., 2013). In common marmosets (Callithrix jacchus), urinary oxytocin levels are correlated between bonded dyads but not between non-bonded dyads, suggesting that it is affiliation between bond partners that affects oxytocin levels (Finkenwirth et al., 2015). Similarly, marital relationship quality is positively correlated with plasma oxytocin levels in humans (Holt-Lunstad et al., 
2014). However, other studies have suggested that oxytocin release following socio-positive behaviors is independent of partner bond strength (Preis et al., 2018; Wittig et al., 2014). As social relationships and bonds are highly differentiated in some group living mammals, determining the behavioral contexts and partners which trigger oxytocin release will be crucial in understanding the dynamics of bond formation and maintenance within groups.

Not only are affiliative interactions able to trigger oxytocin release, but also administration of oxytocin is able to induce a range of socio-positive behaviors (vampire bats, Desmodus rotundus: Carter \& Wilkinson, 2015; dogs: Romero et al., 2014; meerkats, Suricata suricatta: Madden \& Clutton-Brock, 2011; rhesus macaques, Macaca mulatta Chang et al., 2012). In some cases, the socio-positive effects of oxytocin depend on context and social partner (Bartz et al., 2011). For example, intranasal administration of oxytocin increases trust but not if the other person is portrayed as untrustworthy (Mikolajczak et al., 2010). Particularly relevant to social bonds is oxytocin's effect on cooperation. In humans, administered oxytocin promotes generosity and cooperation with members of an in-group but not out-group (De Dreu, 2012; Ten Velden et al., 2017). Similar effects of oxytocin on cooperation are found in naturalistic settings. In particular, chimpanzees who were active participants in inter-group conflicts also had elevated oxytocin levels (Samuni et al., 2017). Other studies involving chimpanzees have also found elevated levels of oxytocin occurring in relation to cooperative hunting (Samuni et al., 2018a) and territorial border patrols (Samuni et al., 2017). These contexts require a high degree of coordination of movement between group members, which oxytocin likely facilitates (Arueti et al., 2013).

Despite our current knowledge of the role of oxytocin in social bond formation, there remain several gaps for understanding its role in social bonding more generally. To date, how partner or context specific oxytocin release contributes to the formation and maintenance of non-reproductive bonds between adults is still largely unclear. Most oxytocin research has been conducted under laboratory conditions in rodents or humans, with much less known on the function of oxytocin in wildlife and under natural, evolutionary relevant conditions. Furthermore, the interplay between oxytocin and the two other main endocrine systems regulating social behaviors, namely the HPG and HPA axis, remains largely unexplored under natural conditions.

\subsubsection{The role of testosterone in social relationships}

Testosterone is the major androgen produced by the hypothalamic-pituitary-gonadal (HPG) axis in male vertebrates (Adkins-Regan, 2005), and is implicated in life-history trade-offs that are relevant to social bonding (van Anders et al., 2011; Wingfield et al., 1990). The challenge hypothesis, originally formulated on studies of seasonally breeding birds, proposes that testosterone levels are primarily elevated in response to reproductive challenges from other males such as territorial intrusions and mate guarding (Wingfield et al., 1990). This hypothesis further proposes that high testosterone levels inhibit paternal care; therefore low levels are 
necessary when care is required, thus mediating a trade-off between mating effort and paternal care (Wingfield et al., 1990).

Studies across vertebrates provided general support for the challenge hypothesis (Archer, 2006; Hirschenhauser \& Oliveira, 2006; Muller, 2017; Oliveira et al., 2002), although there were also several exceptions (Goymann et al., 2019; Lynn, 2008; Moore et al., 2019). For example, in many bird species, it is interactions with receptive females, rather than male-male competition, that are most reliably associated with elevated testosterone levels (Goymann et al., 2019). Inconsistencies were also apparent regarding the relationship between testosterone and paternal care. Although experimental elevations of testosterone levels suppressed paternal care in several bird species (Lynn, 2008), including when elevations were within an individual's reaction norm (Goymann \& Flores, 2017), paternal care remained unaffected in others (Lynn, 2008). In some species, particularly fish, paternal care primarily involves defending nests and is a behavior positively selected for by females (Stiver \& Alonzo, 2009). In such cases there is no trade-off between paternal care and mating effort as high testosterone (or 11-ketotestotserone) levels promote both (Moore et al., 2019; Stiver \& Alonzo, 2009) and resembles the role of testosterone in promoting competition (Moore et al., 2019; Muller, 2017).

The S/P theory (van Anders et al., 2011) attempts to resolve some of the inconsistencies regarding predictions of the challenge hypothesis by defining the trade-off that testosterone mediates more broadly. Specifically, the S/P theory proposes that high testosterone is related to promoting resource acquisition (e.g. in the form of mates or social status) and that it inhibits nurturing behaviors in general rather than paternal care per se (van Anders, 2013; van Anders et al., 2011).

Human parenting (i.e. nurturance) and romantic relationships (i.e. resource acquisition) provide a good example of the predictions of S/P theory within the same species. For example, new human fathers have lower testosterone levels than previous measurements, with greater declines if they are involved in caring for the child (Gettler et al., 2011). Only nurturing responses to infants are associated with lowered testosterone in men (van Anders et al., 2014, 2012). Although testosterone is associated with increased sexual interest in men (Peters et al., 2008; Slatcher et al., 2011; Welling et al., 2008) and facilitates the formation of early-stage sexual relationships (Roney \& Gettler, 2015), men in committed long-term romantic relationships often exhibit lower testosterone levels than their single counterparts (Gray et al., 2004; Roney \& Gettler, 2015; van Anders \& Goldey, 2010). Tellingly, both men and women in long term relationships report lower relationship satisfaction if their partner has high testosterone levels, suggesting that testosterone is incompatible with nurturing behaviors (Edelstein et al., 2014). In line with this interpretation, testosterone inhibits empathy and the reading of others' emotions (Hermans et al., 2006; Ronay \& Carney, 2013). Collectively this research indicates that high testosterone promotes mating effort and is incompatible with satisfactory intimacy in long-term nurturing relationships in humans.

Beyond parental care and romantic relationships, testosterone is also relevant for bonding 
between same-sex adults. Bonds between same-sex adults are characterized by frequent exchanges of affiliative behavior (Massen et al., 2010; Ostner \& Schülke, 2018). Thus, to the extent that testosterone inhibits nurturing behaviors towards infants, it may also be detrimental to same-sex bonding. Same-sex adults have the potential to be reproductive rivals and given testosterone's role in promoting competition, high levels during bonding periods may be detrimental and interfere with the bonding process by cognitively priming individuals to be competitive rather than nurturing (Eisenegger et al., 2011). Therefore, the S/P theory predicts that low testosterone levels would be ideal during bonding periods (van Anders, 2013; van Anders et al., 2011). Overall, testosterone has been less well studied in non-sexual affiliative contexts. Nevertheless, consistent with the S/P theory, a recent study in humans found that lowered testosterone levels were associated with increased feelings of closeness after a friendship formation task between same-sex adult dyads (Ketay et al., 2017). Similarly, male chimpanzees exhibit lower testosterone levels after sharing or receiving meat (Sobolewski et al., 2012), a behavior implicated in the formation of social bonds in this species (Mitani \& Watts, 2001; Wittig et al., 2014). Further studies are needed to determine the pattern holds throughout a wider range of species, particularly those that form strong same-sex bonds in different social systems.

Individuals living in the cross-fire between cooperation and competition are faced with a range of social challenges and - real or perceived - threats. Testosterone enhances competitive ability in individuals but requires additional physiological resources to cope with these social challenges. Here, the HPA axis also plays a role in regulating the physiological responses of individuals as they navigate their social environment.

\subsubsection{The role of glucocorticoids in social relationships}

The HPA axis regulates the release of GCs to help individuals cope with energetic and social challenges (Sapolsky, 2002) and is highly responsive to social relationships. Studies often demonstrate that a strong bond exists between two individuals, because the loss of, or experimental separation from, bonded partners reliably causes increases in GC levels, whereas separation from non-bond partners does not (Cacioppo et al., 2015; Engh et al., 2006; Smith \& Wang, 2012). Other social stressors such as aggressive encounters also generally trigger the release of GCs (Wittig et al., 2016; Young et al., 2014a). However, the presence of a closely bonded social partner is often enough to buffer the GC response to a stressor, and is known as the social buffering effect (Cohen \& Wills, 1985; Hennessy et al., 2009; Hostinar et al., 2014; Wittig et al., 2016; Young et al., 2014a). Even in the absence of stressors, social integration and the ability to count on others for support may help downregulate HPA activity (Brent et al., 2011; Fürtbauer et al., 2014; Rosal et al., 2004; Stocker et al., 2016; Wittig et al., 2016). Conversely, social isolation, even if only perceived, upregulates HPA activity (Cacioppo et al., 2015). Indeed, moderate increases in GC levels serve as motivation to seek affiliative contact in species with complex social systems (Raulo \& Dantzer, 2018), possibly as a means to maintain, repair, or build new bonds. This seeking behavior is of evolutionary significance 
particularly for group-living species as being separated from the group can mean higher risk of predation (Krause \& Ruxton, 2002), a threat ameliorated if GC increases induce the initiation of affiliative contact. Beyond serving as a signal of damaged relationships, bond formation may benefit from lowered GC levels. For example, a decline in cortisol levels from baseline were associated with increased feelings of closeness following a friendship-formation task in humans (Ketay \& Beck, 2017; Ketay et al., 2019).

To summarize, I previously introduced three major hormonal systems in mammals that influence and are influenced by social interactions. Oxytocin is the primary hormone implicated in the formation of a partner preference and social bonds, whereas testosterone and GCs may interact with the oxytocinergic system in such a way as to inhibit or facilitate affiliative interactions and/or the bonding process. I also emphasized the necessity to study these complex interwoven systems under natural conditions. Naturalistic study, however, poses methodological challenges, as often invasive sampling of wild populations is not feasible or impossible due to ethical considerations, especially in the case of threatened species. Therefore it is crucial to be able to measure hormones non-invasively to study the dynamics of hormones and behavior under ecologically valid settings.

\subsection{Considerations for non-invasive hormone measurements}

The advent of methods to measure hormones from non-invasively collected samples such as urine or feces has greatly facilitated research on wildlife endocrinology (Higham, 2016). Urine and fecal samples can be collected from free living animals without the need to disturb them. Non-invasive hormone sampling allows researchers to study how hormones are related to naturally occurring behavior and gives us important new insights that can complement experimental research designs. Furthermore, collection of non-invasive samples is often more practical and raises less ethical concerns than more invasive methods. However, it is important to ensure that hormone measurements from urine and fecal samples truly reflect hormone levels in the blood or brain where directly they act to influence behavior. Measuring oxytocin and steroids from non-invasively collected samples present two different challenges that will be discussed below.

\subsubsection{Central vs. peripheral oxytocin}

Oxytocin is produced by magnocellular and parvocellular neurons in the supraoptic (SON) and paraventricular (PVN) nuclei of the hypothalamus (Landgraf \& Neumann, 2004; Neumann \& Landgraf, 2012). Axonal projections from the hypothalamus transport oxytocin to different areas of the brain for release and also to the pituitary gland where it can be stored and released into the periphery (bloodstream) (Landgraf \& Neumann, 2004).

It is oxytocin released centrally in the brain (rather than in the periphery) that is able to 
exert behavioral effects. However, oxytocin is a large molecule and therefore does not readily cross the blood-brain barrier (Landgraf \& Neumann, 2004). Consequently, there is ongoing debate about whether peripheral measures of oxytocin truly reflect central levels (Macdonald \& Feifel, 2013; Ziegler \& Crockford, 2017). The rationale for peripheral oxytocin reflecting central levels revolves around the potential for coordinated release in the brain and the periphery. It is anatomically possible for coordinated release of central and peripheral oxytocin since axonal projections from the hypothalamus release oxytocin within the brain and posterior pituitary (Landgraf \& Neumann, 2004; Ross et al., 2009). Only a few studies have directly measured coordinated release with mixed evidence, both for (Landgraf et al., 1988; Torner et al., 2017; Wotjak et al., 1998) and against (Amico et al., 1990; Robinson \& Jones, 1982) coordinated release. The context of oxytocin release (e.g. stressors, lactation, mating or affiliation) may play a role on when it is or is not coordinated. Some discrepancies in results may be attributed by use of oxytocin measurement methods that have not been properly validated (Ziegler \& Crockford, 2017). For instance, consensus has shown that blood or urine samples must be extracted before reliable oxytocin measures can be made (Leng \& Sabatier, 2016; McCullough et al., 2013). A number of studies measuring peripheral oxytocin using validated techniques have shown that variation in oxytocin levels reflect predicted changes in relevant behaviors, suggesting that there is indeed coordinated release of central and peripheral oxytocin (reviewed in Crockford et al., 2014). For the intents and purposes of this thesis, using urinary oxytocin as a proxy of central oxytocin is acceptable given that the sample processing procedures, including storage and extraction techniques, have been validated for measuring oxytocin in the urine of nonhuman primates (reviewed in Ziegler, 2018).

\subsubsection{Validation of steroid measurements}

Unlike oxytocin, steroid hormones are relatively small and readily cross the blood-brain barrier; hence peripheral measures of steroids are considered reflective of central levels. The most commonly used methods used to measure steroid hormones are radio- (RIA) and enzyme- (EIA) immunoassays. These assays use antibodies that bind to the hormone of interest, allowing its concentration in a sample to be quantified (Grange et al., 2014). Immunoassays were originally developed and validated to measure steroids from blood samples but have been increasingly applied to measure steroids in non-invasively collected samples such as urine or feces. However, additional validation steps are often required to ensure that immunoassays validated for blood samples also produce reliable results from excreta.

While the antibodies used in immunoassays can be bind with high specificity to a target hormone (e.g. testosterone), they nevertheless also cross-react with other similarly structured compounds. Cross-reactivity with other compounds often negligibly affects measurements from blood samples as steroid hormones circulate in the bloodstream primarily in their free (i.e. unconjugated), biologically active from. However, steroids in urine and feces are often excreted primarily in their metabolized or conjugated forms, relative to the biologically active form (Bahr et al., 2000; Möhle et al., 2002; Palme \& Möstl, 1997). Thus cross-reactivity poses a significant 
issue in hormone measurements from urine or feces. Cross-reactivity is not an issue if similarly structured metabolites originate from the hormone of interest but can be problematic otherwise. Metabolites with different origins than the hormone of interest will confound the immunoassay measurements and the results will not be biologically meaningful (Goymann, 2005; Möhle et al., 2002). The ratio of free hormone to metabolites and conjugated forms can differ greatly across species and between sample matrices within the same species. Thus validation of immunoassays are highly recommended for each new species and the matrix being sampled (Goymann, 2005; Heistermann et al., 2006; Palme, 2019; Touma \& Palme, 2005).

The key to validating an immunoassay is to ensure that it can capture meaningful changes in hormone levels. This can be achieved by pharmacologically inducing the release of the target hormone. For example, the release of corticosteroids may be stimulated by injecting an animal with adrenocorticotrophic hormone, and a GC immunoassay should be able to capture the resulting change in GC levels (e.g. Hirschenhauser et al., 2005; Stevenson et al., 2018). Alternatively, biological validations may be conducted. For example, a testosterone immunoassay should be able to demonstrate testosterone levels of adult males to be higher than those of castrated males, females, or immature males (e.g. Möhle et al., 2002; Pineda-Galindo et al., 2017).

An additional way to validate immunoassay measurements is to compare them to those of liquid chromatography-mass spectrometry (LC-MS) (e.g. Habumuremyi et al., 2014; Gesquiere et al., 2014; Preis et al., 2011). LC-MS differs from immunoassays in that it measures the concentration of hormone in a sample based on its molecular weight and charge (Cross \& Hornshaw, 2016; Hauser et al., 2008a), and does not suffer from issues of cross-reactivity. Therefore, by correlating LC-MS measurements to those of immunoassays we can determine whether immunoassay measurements accurately reflect levels of the target hormone, or if cross-reacting compounds are significantly impacting the immunoassay measurements. Immunoassays can be faster and more cost-effective than LC-MS for projects where only one or two hormones of interest need to be measured (Cross \& Hornshaw, 2016). Thus immunoassays are often the method of choice once properly validated.

\subsection{Study site and species}

To study physiological correlates of social non-reproductive bonding, we chose male Barbary macaques (Macaca sylvanus) as our study species. In the wild, Barbary macaques live in cedar and oak forests in the Atlas and Riff Mountains in Morocco and Algeria (Fooden, 2007). This species lives in multi-male multi-female groups (Thierry et al., 2004), and are seasonal breeders, with a mating season occurring from autumn to winter and a birth season spanning late spring to early summer (Small, 1990). Most relevant to this study, males form strong bonds with other males, which are equitable and last through multiple mating and non-mating seasons (Young et al., 2014b). These social bonds likely have adaptive value. For example, adult male social 
bonds predict the likelihood of coalition formation during the mating season (Berghänel et al., 2011a; Young et al., 2014b), where strongly bonded males are more likely to support each other during conflicts (Young et al., 2014b). Coalition formation can be used as a strategy to increase or maintain dominance rank (Young et al., 2014c), or to enhance mating opportunities (Küster \& Paul, 1992; Young et al., 2013a). Rising in dominance rank via coalition formation could result in higher reproductive success (e.g. Schülke et al., 2010) as mating success is moderately skewed towards high ranking males (Young et al., 2013a). In addition to promoting social support, social bonds amongst Barbary macaques also buffer the physiological impacts of social and environmental stress (Young et al., 2014a), and aid with thermoregulation (Campbell et al., 2018), which can be important for survival chances during harsh winters (McFarland \& Majolo, 2013).

Infants are an integral part of the social lives of male Barbary macaques. Infants facilitate affiliative contact between males particularly as part of triadic male-infant-male interactions (Paul et al., 1996), a behavior proposed to strengthen social bonds between males (Henkel et al., 2010; Paul et al., 1996). Male-infant-male interactions are ritualistic in nature and involve two males sitting in body contact, holding an infant between them, displaying affiliative facial signals (i.e. teeth chattering), and often inspecting the infant's genitals (Deag, 1980; Deag \& Crook, 1971). Male-infant-male interactions primarily occur during the non-mating season and predict coalition formation in the following mating season (Berghänel et al., 2011a). An alternative function of male-infant-male interactions is that they are a form of "agonistic buffering" (not to be confused with social buffering) to reduce tensions between males (Deag \& Crook, 1971; Paul et al., 1996). In support of this hypothesis, rates of male-infant-male interactions are higher during tense feeding situations, whereas other types of affiliation, such as grooming, go down (Paul et al., 1996). Independent of triadic male-infant-male interactions males also care for infants by huddling, carrying, or grooming them (Whitten, 1987). Males prefer to care for infants based the likelihood of paternity inferred from their past mating success with the mother (Kubenova et al., 2019b), although they are often not the genetic father of their preferred infant (Ménard et al., 2001; Paul et al., 1996).

The study animals were 14 adult males belonging to one of three groups (group C) of Barbary macaques living in semi-free ranging conditions at Affenberg Salem, Germany (de Turckheim \& Merz, 1984). In this park, monkeys stay outdoors year-round in 14.5 ha of enclosed forest (de Turckheim \& Merz, 1984) under social and climatic conditions resembling their natural habitat. Females are philopatric, while males can and do migrate between groups. The monkeys were provisioned daily with fruits, vegetables, and grains, had ad libitum access to monkey chow and water, and also foraged on natural food sources such as insects, mushrooms, and plant shoots. 


\subsection{Thesis aims}

In this thesis I investigated the three major neuroendocrine axes responsible for regulating animal social behavior - the oxytocinergic system, the HPG, and HPA axes - in relation to social bonding in adult male Barbary macaques. A primary aim was to test the $\mathrm{S} / \mathrm{P}$ theory that predicts that the ideal physiological conditions for the formation of social bonds involve high oxytocin levels coupled with low testosterone levels. Most studies on the formation of social bonds have focused on parent-offspring and pair bonds. Only a few studies have investigated the role of oxytocin in the formation platonic social bonds between adults, particularly in natural settings, despite the clear fitness benefits that they provide. Even fewer studies have looked at the role of steroid hormones (i.e testosterone and GCs) in the context of bond formation between same-sex adults. In addition, I also investigate the function of the HPA axis to draw a more complete picture due to its high responsiveness to social interactions and potential for interaction with the oxytocinergic system and HPG axis.

In study 1 (chapter 2), I conducted validations assessing the suitability of three different enzyme immunoassays, two testosterone and one cortisol, to assess gonadal and adrenocortical activity, respectively, in the urine of adult and immature male Barbary macaques. First, I validated steroid measurements by comparing EIA measurements with those made by LC-MS to assess whether cross-reactivity of the antibody with metabolites other than the target hormone would confound results. Finally, I conducted a biological validation of testosterone by comparing levels of adult males to those of immature males.

In study 2 (chapter 3), I focused on the role of oxytocin in bond formation and maintenance between adults outside of a pair bond context. My primary aim was to test whether urinary oxytocin levels were elevated following affiliative social behaviors that promote bonding between adults (i.e. grooming, male-infant-male interactions) and if such elevations were dependent on the existing bond strength between the interaction partners. A secondary aim was to investigate the role of oxytocin on cooperation in a natural setting, testing whether the occurrence of affiliative interactions, and thus putatively elevated oxytocin levels, would increase the probability of supporting another individual in an agonistic conflict.

In study 3 (chapter 4), I built upon study 1 by testing the steroid component of the $\mathrm{S} / \mathrm{P}$ theory, focusing on the role of testosterone and cortisol (the primary GC in Barbary macaques) on the formation of same-sex adult bonds and male infant care. Here, I predicted a negative relationship between testosterone with male bonding behavior (i.e. male-infant-male interactions) and nurturing infant care (i.e. huddling, carrying, grooming). As male bonds are linked to social support, I also investigated the relationship of cortisol with these behaviors for a more complete picture. I predicted a negative relationship between cortisol and male-infant-male interactions. A previous study on male Barbary macaques found a positive relationship between fecal GC levels and infant handling (Henkel et al., 2010), so here I similarly predict a positive relationship between cortisol levels and infant care. 


\section{Chapter 2}

\section{Measuring urinary cortisol and testosterone levels in male}

\section{Barbary macaques: A comparison of EIA and LC-MS}

Alan V. Rincon ${ }^{\mathrm{a}, \mathrm{b}}$, Julia Ostner ${ }^{\mathrm{a}, \mathrm{b}, \mathrm{c}}$, Michael Heistermann ${ }^{\mathrm{d}+}$, and Tobias Deschner ${ }^{\mathrm{e}+}$

${ }^{a}$ Department of Behavioral Ecology, Johann-Friedrich-Blumenbach Institute for Zoology and Anthropology, University of Goettingen, Goettingen, Germany

${ }^{b}$ Leibniz ScienceCampus Primate Cognition, Goettingen, Germany

${ }^{c}$ Research Group Social Evolution in Primates, German Primate Center, Leibniz Institute for Primate Research, Goettingen, Germany

${ }^{d}$ Endocrinology Laboratory, German Primate Center, Leibniz Institute for Primate Research, Goettingen, Germany

${ }^{e}$ Department of Primatology, Max Planck Institute for Evolutionary Anthropology, Leipzig, Germany

+ These authors contributed equally to this study as senior authors

General and Comparative Endocrinology, 281, 117-125 (2019). https://doi.org/10.1016/j.ygcen. 2019.05.017. 


\section{Abstract}

The development of methods to quantify hormones from non-invasively collected samples such as urine or feces has facilitated endocrinology research on wild-living animals. To ensure that hormone measurements are biologically meaningful, method validations are strongly recommended for each new species or sample matrix. Our aim was to validate three commonly used enzyme immunoassays (EIA), one for analysis of cortisol and two for analysis of testosterone, to assess adrenocortical and gonadal endocrine activity, respectively, from the urine of male Barbary macaques. We compared EIA and liquid chromatography-mass spectrometry (LC-MS) results to determine if the EIA measurements truly reflect levels of the target hormone and to determine if antibody cross-reactivities with other steroids were potentially confounding results. Furthermore, we conducted a biological validation of testosterone to ensure that both EIA and LC-MS were able to capture physiologically meaningful differences in hormone levels. We found that cortisol measured by EIA correlated strongly with cortisol measured by LC-MS in both adult and immature males, without the need for deconjugation of steroids in the urine. Both testosterone EIAs correlated strongly with LC-MS in adult males, but only if steroids in the urine were deconjugated by enzymatic hydrolysis prior to analysis. However, in immature males, EIA and LC-MS results did not correlate significantly. Further correlation analyses suggest this is likely due to cross-reactivity of the testosterone antibodies with other adrenal steroids such as cortisol, DHEA, and likely others, which are present at much higher concentrations relative to testosterone in immature males. Testosterone levels were significantly higher in adult compared to immature males as measured by LC-MS but not as measured by EIA. Taken together, our results suggest that the testosterone EIAs are suitable to assess gonadal activity in adult but not immature males, and only if a hydrolysis of the urine is conducted prior to analysis.

Keywords: Testosterone, Cortisol, EIA, LC-MS, Urine, Barbary macaque 


\section{$2.1 \quad$ Introduction}

Steroid hormones facilitate a range of behaviors and developmental changes in animals. For example, glucocorticoids help to mobilize energy reserves and respond adaptively to environmental and social stressors (Sapolsky, 2002). Androgens, such as testosterone (T), the predominant male sex-hormone, promote the production of sperm, development of secondary sexual characteristics, and male reproductive competition (Wingfield et al., 1990). To study steroid hormones in wild-living animals, behavioral ecologists and wildlife endocrinologists are increasingly measuring hormone levels non-invasively, usually via the analysis of excreted hormone metabolites in urine or feces (e.g. Wasser et al., 2000, 1988; Brown et al., 1994; Robbins \& Czekala, 1997; also see Higham, 2016 for a review, including more references and information on the history of non-invasive hormone measurements). This is in part due to the practical and ethical advantages these methods have compared to the traditional approach of measuring hormones invasively from blood. Notably, urine and fecal samples can be collected repeatedly over time without the need to capture or disturb the animal.

The most commonly used methods to quantify hormone concentrations in the various matrices are radio- (RIA) and enzyme- (EIA) immunoassays. These methods rely on the use of antibodies that bind to the hormone (or their metabolites) of interest, thus allowing for its concentration to be quantified in a sample (Grange et al., 2014). Despite their specificity, antibodies used in immunoassays may cross-react with other structurally similar metabolites. In blood, where native unconjugated steroid hormones circulate at much higher concentrations than their metabolites, the impact of such cross-reactivities is often negligible. However, steroids are extensively metabolized in the liver and/or by gut bacteria (Taylor, 1971), and as a result, the concentration of the native unconjugated hormone excreted in urine or feces is usually very low relative to its metabolites or conjugated forms (Bahr et al., 2000; Möhle et al., 2002; Palme \& Möstl, 1997). Thus the impact of cross-reactivity is usually more pronounced in the analysis of urine or fecal samples than of blood samples. Immunoassays for the measurement of testosterone often lack the needed antibody specificity and thus may be of limited use for direct measurement of testosterone in urine (Venturelli et al., 1995). Nevertheless, if the cross-reacting metabolites originate from the parent hormone of interest, the signal detected by the assay may still be biologically meaningful. However, if the cross-reacting compounds measured by the antibody used originate from hormones with different biological functions, then results are confounded and may be uninterpretable. For example, some testosterone immunoassays co-measure androgen metabolites of non-gonadal origin (likely from dehydroepiandrosterone (DHEA), which is of adrenal origin) to such an extent, that they fail to find the predicted difference in testosterone levels between males and females (Goymann, 2005; Möhle et al., 2002). Similarly, two out of four glucocorticoid EIAs showed substantial cross-reactivity with testosterone metabolites in the urine and feces of male African elephants (Loxodonta africana), potentially leading to a confound when applied for the assessment of adreonocortical activity in this species (Ganswindt et al., 2003).

As steroids are primarily excreted in their conjugated form in urine (Bahr et al., 2000; Möhle 
et al., 2002; Ziegler et al., 2000), results from hormone-specific assays designed to measure the free (i.e. unconjugated) native hormone in blood may be improved by first deconjugating the steroids via hydrolysis and/or solvolysis (Hauser et al., 2008a; Venturelli et al., 1995), thus increasing the ratio of free native hormone in the sample. Failure to do so may produce inconsistent results. For example, two studies on wild chimpanzees (Pan troglodytes) tested for a relationship between dominance rank and urinary testosterone levels. One study found a significant positive correlation, where high ranking males had higher testosterone (Muller \& Wrangham, 2004), whereas the other study did not find a significant relationship (Sobolewski et al., 2013). While this could be a true difference between populations, methodological differences in hormone analysis could also account for this discrepancy. The key difference is that in the former study, steroids in the urine were deconjugated via hydrolysis prior to analysis (Muller \& Wrangham, 2004), but in the latter study, testosterone was analyzed from unprocessed urine samples (Sobolewski et al., 2013). Considering that the vast majority of testosterone is excreted as glucuronide conjugates in chimpanzee urine (Möhle et al., 2002), it is unclear whether using a testosterone EIA on non-hydrolyzed urine produces biologically meaningful results, particularly when cross-reactivity of the antibody with conjugated testosterone is not known or very low.

Given the discrepancies mentioned above, it is of paramount importance to validate hormone assay methods prior to their application in non-invasively collected samples (Heistermann et al., 2006; Möhle et al., 2002; Touma \& Palme, 2005; Ziegler et al., 2000). Furthermore, due to variation in hormone metabolism, method validations are strongly recommended for each new species, sex, or sample matrix (Buchanan \& Goldsmith, 2004; Goymann, 2005; Heistermann et al., 2006; Palme, 2019; Touma \& Palme, 2005).

Traditionally, two methods have been used to validate measurements of immunoassays. First, radioinfusion studies work by injecting a small amount of radio-labelled hormone into the animal and collecting all subsequent excreta. Since the injected hormone is radio-labelled, researchers are able to deduce the time-lag to hormone excretion, the metabolism pathway, and whether an antibody really cross-reacts with the target hormone (Goymann, 2005; Palme, 2019; Wasser et al., 2000). Second, physiological validations of steroid hormone measurements may be conducted by pharmacologically inducing their release in the body, then checking if the immunoassay is able to capture the resulting change in hormone levels (Goymann, 2005; Heistermann et al., 2006; Kretzschmar et al., 2004; Palme, 2019; Wasser et al., 2000). While these methods provide invaluable information, one limitation is that they are invasive in nature and usually carried out in captivity, which may not always be practical.

One way to ensure that non-invasive methods can capture natural variations in hormone levels is to conduct biological validations. For example, a testosterone assay should be able to differentiate levels of adult males from those of immature males or females (Möhle et al., 2002; Pineda-Galindo et al., 2017), a glucocorticoid assay should be able to detect rises following putatively stressful events such as translocation, capture and restraint (Pineda-Galindo et al., 2017; Touma \& Palme, 2005), and estrogen or progesterone assays should be able to detect changes in female reproductive condition (i.e. menstrual cycle, pregnancy: Pineda-Galindo et 
al., 2017; Fieß et al., 1999).

Liquid chromatography-mass spectrometry (LC-MS) can also be used as a tool to validate immunoassay measurements (Gesquiere et al., 2014; Habumuremyi et al., 2014; Preis et al., 2011). In contrast to immunoassays, LC-MS does not use antibodies for hormone detection, but allows for highly specific measurements of hormones in samples based on their molecular weight and charge (Cross \& Hornshaw, 2016; Hauser et al., 2008a), thus avoiding any confounding effects of antibody cross-reactivity. Therefore, comparing immunoassay measurements to LC-MS is a useful way to deduce if measurements from an immunoassay indeed reflect the concentration of the target hormone and which cross-reacting metabolites may be potentially confounding results (Gesquiere et al., 2014; Habumuremyi et al., 2014; Preis et al., 2011). Both immunoassays and LC-MS may be applied to non-invasively collected samples and the comparison of their measurements offer a useful alternative when more invasive validation methods are not feasible or desirable. Once sufficiently validated, immunoassays have the advantage of being cheaper and having a higher throughput than LC-MS (Cross \& Hornshaw, 2016). Therefore, as long as the focus of a study is on a single hormone, routine hormone measurements with immunoassays are more practical for end-users in terms of cost and resourcing especially in labs that may not have access to LC-MS.

In this study, we aimed to determine whether three commonly used EIAs, one cortisol and two testosterone, previously used in other nonhuman primates (Bahr et al., 2000; Möhle et al., 2002; Sobolewski et al., 2013) were suitable to assess adrenocortical and gonadal activity, respectively, in the urine of male Barbary macaques (Macaca sylvanus). In this species, validations have been conducted for assays measuring glucocorticoid (Heistermann et al., 2006; Young et al., 2014a) and androgen (Rincon et al., 2017) metabolite levels in fecal samples. Only one study has measured cortisol levels in the urine of (female) Barbary macaques (Sonnweber et al., 2015), although, to our knowledge, no validation has yet been conducted for urinary cortisol and testosterone measurements in this species. First, we examined the pattern of conjugation of cortisol and testosterone in the urine of adult and immature male Barbary macaques. Then, we correlated the LC-MS cortisol and testosterone measurements to their respective EIA measurements from unprocessed urine to determine whether they would closely match, or whether deconjugation steps might be necessary prior to using EIA in order to improve results (Al-Dujaili, 2006). Thus, if these results did not correlate significantly, we then performed a deconjugation step (Venturelli et al., 1995; Ziegler et al., 2000) to see if the correlation improved and by this assessed the general suitability of the immunoassays used (c.f. Al-Dujaili, 2006). The deconjugation step performed (hydrolysis or solvolysis) was chosen based on the pattern of conjugation. To determine any potential influence of cross-reactivity and potential co-measurement of steroid metabolites of different origins on our EIA measurements, we correlated measurements from the three EIAs to cortisol, testosterone and DHEA as measured by LC-MS. To complement the methodological testosterone validations, we additionally performed a biological validation of testosterone by comparing levels of adult males to those of immature males. We predicted that testosterone levels would be higher in adult males compared to immature males (Rincon et al., 2017). 


\section{$2.2 \quad$ Materials and Methods}

\subsubsection{Ethical statement}

All methods used in this study were non-invasive (i.e. animals were not handled at all) and followed the Animal Behaviour Society's guidelines for the ethical treatment of animals in behavioral research and teaching.

\subsubsection{Study site and animals}

Study subjects belonged to one out of three groups of Barbary macaques living in semi-free ranging conditions in 14.5 ha. of enclosed forest at Affenberg Salem, Germany (de Turckheim \& Merz, 1984). They are provisioned daily with fruits, vegetables, grains and have ad libitum access to water and monkey chow. The study group (group C) consisted of 13-14 adult males (one male died during the study period), 20 adult females, 2 large sub-adult males, 8 immature males, 10 immature females and 1 newborn infant male. All members of the group were individually recognizable by observers based on a combination of distinctive individual characteristics, such as facial spots and scars, canine size, body size, coat color and quality as well as unique tattoos on the inner right thigh.

\subsubsection{Sample collection}

A total of 62 urine samples were collected between April and October 2016 from a total of 21 males, including 30 samples from 13 adults ( 7 to 25 years old), and 32 samples from 8 immature individuals ( 1 to 4.5 years old). When monkeys were seen to urinate, the urine was caught with a plastic bag when possible or collected from leaves, branches, rocks or the ground by using a disposable pipette or salivette (Salivette Cortisol, Sarstedt, Nümbrecht, Germany; see Danish et al., 2015; Müller et al., 2017). Urine samples contaminated with feces were not collected. Urine samples collected by pipette were transferred to $2 \mathrm{ml}$ cryotubes. Both samples stored in cryotubes and salivettes were kept in a thermos filled with ice while in the field. At the end of the day, urine was recovered from the salivettes by centrifugation using an electric centrifuge and also transferred to $2 \mathrm{ml}$ cryotubes. All samples were then stored in a freezer at $-20^{\circ} \mathrm{C}$. When data collection was complete, samples were transported in containers with dry ice to the endocrinology laboratory where they were once again kept frozen at $-20^{\circ} \mathrm{C}$ until hormone analysis. 


\subsubsection{Hormone analysis}

\subsubsection{Deconjugation and extraction of steroids}

The deconjugation and extraction of steroids for LC-MS analysis followed a modified version of a protocol previously described (Hauser et al., 2008a; Preis et al., 2011). We used 20 ul urine for analysis. To each urine sample we added $50 \mu \mathrm{l}$ internal standard mixture, to control for losses during extraction and purification, and matrix effects on ionisation of MS measurements. Internal standard mixtures contained $2 \mathrm{ng} / \mathrm{ml}$ each of testosterone-d 3 and estrone-d4 from Sigma-Aldrich (St. Louis, MO, USA), progesterone-d9 from CDN Isotopes (Point-Claire, QC, Canada), and prednisolone from Steraloids (Newport, Rhode Island, USA). However, for this study, only values for testosterone-d3 were used for the correction of testosterone and DHEA and values for prednisolone were used for the correction of cortisol. Steroid glucuronides were hydrolyzed by mixing $20 \mu \mathrm{l}$ urine of each sample with $10 \mu \mathrm{l} \beta$-Glucuronidase (K12 strain Escherichia coli, Prod. No. BGALS-RO, Sigma-Aldrich), $50 \mu$ internal standard mixture (see above), and $250 \mu \mathrm{l} 0.05 \mathrm{M}$ phosphate buffer ( $\mathrm{pH} 6.5$ ) and incubating in a water bath at $55^{\circ} \mathrm{C}$ for $30 \mathrm{~min}$. Extracts were purified by solid phase extractions (Chromabond HR-X, 30mg, 1ml, Macherey-Nagel, Dueren, Germany) (Hauser et al., 2008a). Afterwards, steroid sulfates were cleaved by solvolysis: residues of the aqueous layer were resolved in $500 \mu \mathrm{l}$ of methanol and $2.5 \mathrm{ml}$ ethyl acetate $/ \mathrm{H}_{2} \mathrm{SO}_{4}$ (250 ml ethyl acetate $/ 200 \mathrm{mg} \mathrm{H}_{2} \mathrm{SO}_{4}, 98 \%$ ). This mix was then incubated for 15 minutes at $55^{\circ} \mathrm{C}$ in a water bath. Following incubation, $150 \mu \mathrm{l} 1 \mathrm{M} \mathrm{KOH}$ was added to the solvolyzed samples, vortexed and then centrifuged for $3 \mathrm{~min}$ at $870 \mathrm{~g}$. The solution was evaporated to dryness at $45^{\circ} \mathrm{C}$ under air. This solvolysis step was carried out only for the measurements of steroids by LC-MS (and not EIA). Extraction of steroids was carried out with $5 \mathrm{ml}$ tert. butyl methyl ether (TBME), evaporated and reconstituted in $100 \mu \mathrm{l}$ of $30 \%$ acetonitrile. Extraction efficiencies for the LC-MS measurements were $81.9 \%$ for cortisol, $77.4 \%$ for testosterone and $62.7 \%$ for DHEA (Hauser et al., 2008a). HPLC grade water and $\mathrm{H}_{2} \mathrm{SO}_{4}$ were purchased from J.T. Baker (Mallinckrodt Baker, Phillipsburg, NJ, USA). Potassium carbonate $\left(\mathrm{K}_{2} \mathrm{CO}_{3}\right)$ was purchased from Merck (Kenilworth, NJ, USA). Ammonium acetate was purchased from Sigma-Aldrich. Methanol, ethyl acetate, monosodium phosphate, disodium phosphate, TBME, KOH, and UPLC grade acetonitrile were purchased from Roth (Karlsruhe, Germany).

To determine the pattern of conjugation, we first extracted the urine to obtain the unconjugated fraction, then performed a hydrolysis on the aqueous phase to obtain the glucuronide fraction and finally performed a solvolysis on the remaining aqueous phase to obtain the sulfate fraction. The concentration of each fraction was determined by LC-MS and summed to provide a measure of the total concentration of hormone (cortisol or testosterone). The pattern of conjugation is reported as the percentage of each fraction of the total sum.

To measure testosterone in the urine via EIA, we performed an enzymatic hydrolysis and extraction as described in section 2.2.4.1, but without adding the internal LC-MS standard mixture to the samples. Furthermore, we decided not to perform a sovolysis on the samples. 
This was because we found that in both adult and immature individuals, consistently only a small proportion of testosterone was excreted as sulfates (see Results, Fig. 2.1). We are confident that disregarding the small amount of sulfated testosterone excreted does not influence the across individual/sample variation in testosterone concentrations that we were interested in. Therefore, for the routine measurement of samples with EIA we skipped the solvolysis step to save on time and costs. To assess the efficiency of the combined hydrolysis and extraction procedure, we prepared a stock solution of testosterone-glucuronide (Art. No. T-2000; Merck KGaA, Darmstadt, Germany) with a concentration of $50 \mathrm{pg} / \mathrm{\mu l}$ in $\mathrm{MeOH} / \mathrm{H}_{2} \mathrm{O}(50 / 50)$. Aliquots of 0.4 $\mathrm{ml}$ each of this stock solution were stored at $-20^{\circ} \mathrm{C}$ until used in the hydrolysis/extraction process as separate controls (i.e. using $20 \mathrm{pl}$ of control solution instead of $20 \mathrm{\mu l}$ of urine). Hydrolysis and extraction of controls were performed as described in section 2.2.4.1 (see above). Testosterone levels were measured from hydrolyzed controls using T-EIA I as described in section 2.2.4.3 (see below). To determine the combined hydrolysis/extraction efficiency, testosterone values measured in the controls were divided by the added amount and expressed as a percentage. The combined hydrolysis/extraction efficiencies ranged from $68 \%$ to $82 \%$ recovery $(\mathrm{N}=11$, mean \pm SD: $76.6 \pm 4.7 \%)$.

\subsubsection{LC-MS analysis}

LC measurements were carried out using a Waters Acquity UPLC separation module equipped with a binary solvent manager and a column oven (Waters, Milford, MA, USA) and separation was performed on a Waters Acquity BEH C18 column ( 2.1 x $100 \mathrm{~mm}, 1.7 \mu \mathrm{m}$ particle diameter). Eluent A was water with $0.1 \%$ formic acid and Eluent B was acetonitrile. MS analyses were carried out on a Waters XEVO TQ-S tandem quadrupole mass spectrometer (Micromass, Manchester, UK) with an electro spray interface (ESI) in positive mode (Wessling et al., 2018). The quantitative analysis by LC-MS was realized in the range of $0.03-100 \mathrm{ng} / \mathrm{\mu l}$ for cortisol and testosterone and 0.5- $100 \mathrm{ng} / \mathrm{\mu l}$ for DHEA (Hauser et al., 2008a, 2008b). One sample was excluded due to internal standard loss of $>80 \%$. For all other samples $(\mathrm{N}=61)$, internal standard loss was $<45 \%$. For each sample analyzed, hormone concentrations were corrected for individual losses. We examined LC-MS data with MassLynx (Version 4.1; QuanLynx-Software).

\subsubsection{EIA}

Immunoreactive urinary cortisol (iuCort ${ }_{\text {EIA }}$ ) concentrations were determined in unprocessed diluted urine by microtiter plate enzyme immunoassay using an antiserum against cortisol-3-CMO-BSA and biotinylated cortisol as enzyme conjugate (Palme \& Möstl, 1997). Prior to analysis, samples were diluted 1:100 to 1:12,800 (to ensure concentrations fell in the linear range of the standard curve) in assay buffer and duplicate 50 $\mathrm{\mu l}$ aliquots of diluted samples and cortisol standard (50 $\mathrm{\mu l}, 0.6-40 \mathrm{pg} / 50 \mathrm{\mu l})$ were combined with labelled cortisol (50 pl) and antiserum $(50 \mathrm{\mu l})$ and incubated overnight at $4^{\circ} \mathrm{C}$. After incubation, the plates were washed four times (plate washer model 405 TS, BioTek Instruments GmbH; Bad Friedrichshall, 
Germany), $150 \mathrm{\mu l}$ (667 ng) of streptavidin-peroxidase (S5512; Sigma-Aldrich Chemie GmbH, Deisenhofen, Germany) in assay buffer was added to each well and the plates incubated at room temperature in the dark for $60 \mathrm{~min}$ and then washed again four times. TMB substrate solution (100 $\mu$ l; 1-Step Ultra TMB, Thermo Fisher Scientific Inc., Rockford, USA) was subsequently added and the plates incubated at room temperature in the dark for another 45 - 60 min. The enzyme reaction was finally stopped by adding $50 \mu \mathrm{l}$ of $2 \mathrm{M} \mathrm{H}_{2} \mathrm{SO}_{4}$ to each well and absorbance measured at $450 \mathrm{~nm}$ (reference $630 \mathrm{~nm}$ ) in a plate spectrophotometer (EL 808, BioTek Instruments GmbH; Bad Friedrichshall, Germany). Cross-reactivity of the antibody is given in Palme \& Möstl (1997). Serial dilutions of samples showed displacement curves that run parallel to the respective standard curve. Assay sensitivity at $90 \%$ binding was $0.6 \mathrm{pg}$. Intra-assay coefficients of variation $(\mathrm{CV})$ of high and low value quality controls (pool samples of standard preparations) were $5.8 \%$ (high) and $7.7 \%$ (low) while respective figures for inter-assay CVs were $7.4 \%$ (high, $\mathrm{N}=6$ assays) and $6.2 \%$ (low, $\mathrm{N}=6$ assays).

For measurement of immunoreactive urinary testosterone (iuT $\mathrm{TIA}_{\mathrm{A}}$ ) in all sample types (diluted unprocessed urine samples, and samples following extraction, and hydrolysis), we generally applied a testosterone EIA (T-EIA I) using an antiserum that was purchased from Rupert Palme (University of Veterinary Medicine, Vienna, Austria; Palme \& Möstl, 1994). In addition, a subset of samples was also measured with a second testosterone EIA (T-EIA II) using an antiserum (R156/7) purchased from late Coralie Munro (Clinical Endocrinology Laboratory, UC Davis, USA). The latter was done in order to examine whether different EIAs designed for the measurement of testosterone would generally provide the same or different results when applied to the measurement of testosterone metabolites in the urine of Barbary macaques. Antibodies were both raised in rabbits against testosterone-3-CMO-BSA (T-EIA I) and testosterone-6-CMO-BSA (T-EIA II). For the assay, in brief, $50 \mu$ l aliquots of diluted samples (1:20 to 1:2500; to ensure concentrations fell in the linear range of the standard curve) and testosterone standard ( $50 \mu \mathrm{l}, 0.31$ - $20 \mathrm{pg} / 50 \mu \mathrm{l})$ were combined with HRP-labelled testosterone $(50 \mathrm{\mu l})$ and antiserum $(50 \mu \mathrm{l})$ and incubated overnight at $4^{\circ} \mathrm{C}$. After incubation, the plates were washed four times, after which TMB substrate solution (100 $\mu$; 1-Step Ultra TMB, Thermo Fisher Scientific Inc., Rockford, USA) was added and the plates incubated at room temperature in the dark for another 45 - $60 \mathrm{~min}$. The enzyme reaction was finally stopped by adding 50 $\mu \mathrm{l}$ of $2 \mathrm{M} \mathrm{H}_{2} \mathrm{SO}_{4}$ to each well and absorbance measured at $450 \mathrm{~nm}$ (reference $630 \mathrm{~nm}$ ) in a spectrophotometer (see above).

Cross-reactivities for T-EIA I were reported in Palme \& Möstl (1994), while those for T-EIA II were reported in Kersey et al. (2010). Serial dilutions of samples of both diluted neat urine and urine following hydrolysis and extraction showed displacement curves that run parallel to the respective standard curve in both assays. Assay sensitivities at $90 \%$ binding were $0.3 \mathrm{pg}$ for both EIAs. Intra-assay coefficients of variation (CV) of high and low value quality controls (pool samples of standard preparations) were $<10 \%$ in both assays. Respective inter-assay CVs were $9.0 \%$ (high, $\mathrm{N}=10$ assays) and $10.7 \%$ (low, $\mathrm{N}=10$ assays) for T-EIA I and $8.5 \%$ (high, $\mathrm{N}=3$ assays) and $14.5 \%$ (low, $\mathrm{N}=3$ assays) for T-EIA II. 
Urinary steroid concentrations (from both EIA and LC-MS) were corrected for creatinine, measured as described by Bahr et al. (2000), to account for differences in urine concentration and are expressed as ng/mg creatinine (ng/mg Cr).

In this paper, we refer to the urinary cortisol, testosterone and DHEA measurements by LC-MS as uCort ${ }_{\mathrm{LC}-\mathrm{MS}}, \mathrm{uT}_{\mathrm{LC}-\mathrm{MS}}$ and $\mathrm{uDHEA}_{\mathrm{LC}-\mathrm{MS}}$, respectively. By contrast, we refer to immunoreactive urinary cortisol and testosterone measurements by EIA as iuCort EIA $_{\text {and }}$ iu $\mathrm{T}_{\mathrm{EIA}}$, respectively. We would like to emphasize the fact that while LC-MS accurately quantifies the levels of each hormone in urine, the measurements from EIA are influenced by potential cross-reactivity of the antibody with other compounds.

\subsubsection{Statistical analysis}

All analyses were conducted in R statistical software version 3.5.1 (R Core Team, 2018). For all analyses we used non-parametric tests and computed exact p-values where appropriate (Mundry \& Fischer, 1998).

To compare hormone levels measured by EIA and LC-MS and to detect any potential cross-reactivity in the EIAs used, we correlated EIA and LC-MS results using a Spearman's rank correlation. We used a bootstrapping procedure to avoid pseudo-replication due to having multiple samples from each individual. Using an $\mathrm{R}$ script, we randomly selected one urine sample from each male and calculated a correlation coefficient across all males. We repeated this procedure 1,000 times, saving all correlation coefficients. If we only had one sample for an individual, then this sample was re-used in all correlations. To avoid the issue of multiple testing we did not calculate p-values. Instead, to infer significance of the correlations, we calculated $95 \%$ confidence interval of all correlation coefficients and if the interval did not include 0 , we deemed the correlation to be significant. We report the mean correlation coefficient (rho) from all correlations.

To compare testosterone levels between adult and immature males, we used a Mann-Whitney U test. Mean hormone levels per individual were used when we had multiple samples from the same individual. The significance value was set to $\mathrm{p}<0.05$.

\subsection{Results}

The pattern of conjugation of cortisol and testosterone, as revealed by LC-MS, was very similar between adult and immature males. Both steroids were primarily excreted as glucuronides with sulfates making up only a small portion (Fig. 2.1). For cortisol, a substantial proportion was also excreted unconjugated, i.e., in free form, while free testosterone made up only a small portion of the total amount of urinary testosterone (Fig. 2.1). 


\subsubsection{Steroid levels in urine}

For both age classes, uCort $_{\mathrm{LC}-\mathrm{MS}}$ was the most abundant, followed by $\mathrm{uDHEA}_{\mathrm{LC}-\mathrm{MS}}$, and then $\mathrm{uT}_{\mathrm{LC}-\mathrm{MS}}$ (Fig. 2.2).

Absolute concentrations of iuCort EIA $_{\text {and }}$ au $\mathrm{T}_{\text {EIA }}$ were higher than Cort $_{\mathrm{LC}-\mathrm{MS}}$ and $\mathrm{uT}_{\mathrm{LC}-\mathrm{MS}}$, respectively (cortisol adults: 2 times, cortisol immature: 3 times; testosterone adults EIA I: 4 times and EIA II: 5 times, immature EIA I: 19 times, and EIA II: 18 times; Fig. 2.2). Absolute concentrations of $i \mathrm{~T}_{\text {EIA-I }}$ and $i u \mathrm{~T}_{\text {EIA-II }}$ were very similar in both adult and immature males (Fig. 2.2).

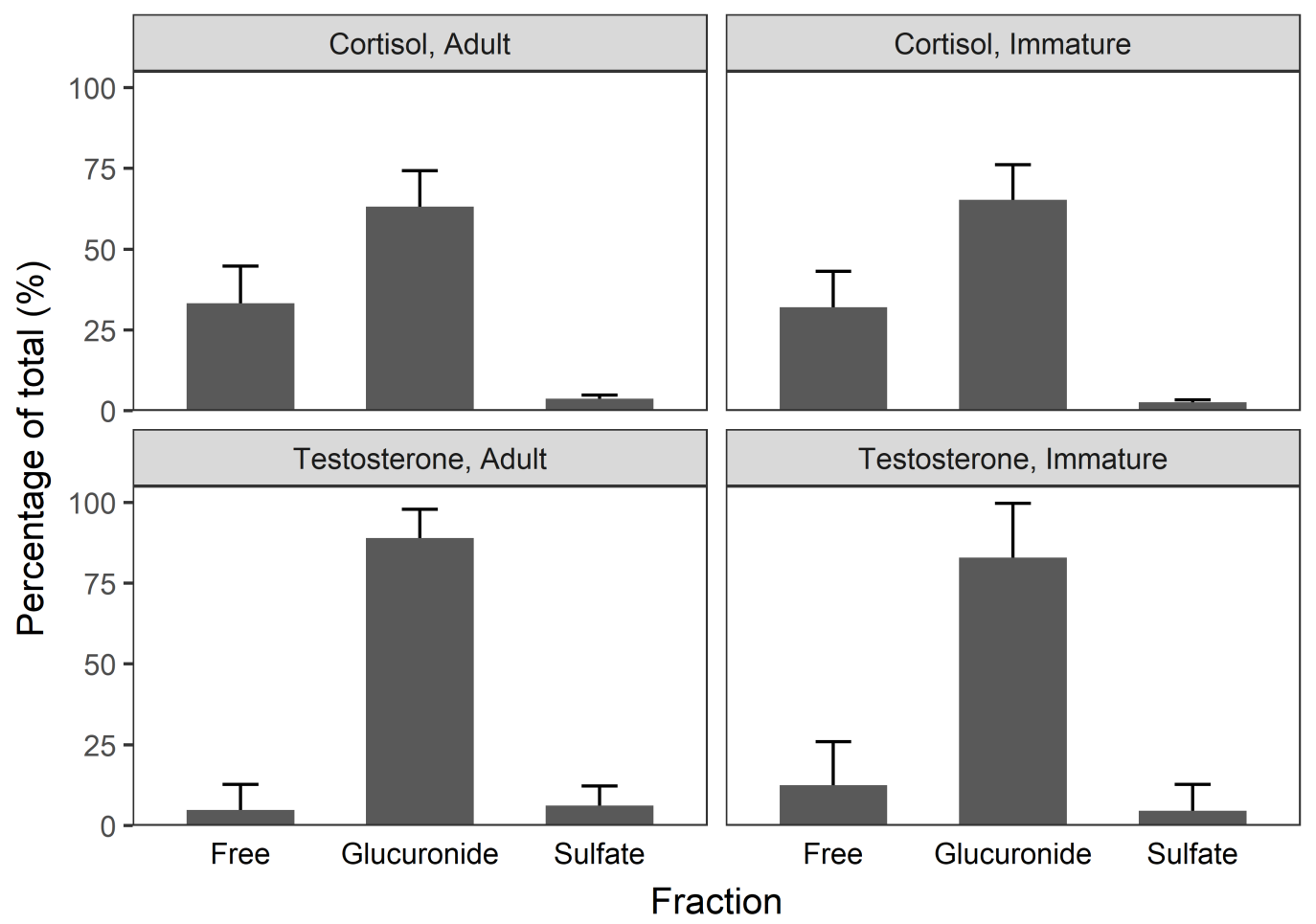

Figure 2.1: Proportion of cortisol and testosterone excreted in conjugated (glucuronide or sulfate) and unconjugated (free) form in male Barbary macaque urine, as measured by LC-MS. $\mathrm{N}=13$ adult, $\mathrm{N}=8$ immature males. Error bars indicate standard deviation.

\subsubsection{Correlations between urinary steroids measured by LC-MS and EIA}

We found that iuCort ${ }_{\mathrm{EIA}}$ as measured from unprocessed urine correlated strongly, positively and significantly with uCort $_{\mathrm{LC}-\mathrm{MS}}$ in both adult and immature males (Table 2.1 and Table 2.2).

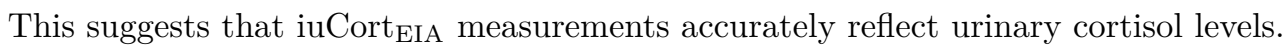

We found that $\mathrm{iuT}_{\mathrm{EIA}-\mathrm{I}}$ and $\mathrm{iuT}_{\mathrm{EIA}-\mathrm{II}}$ as measured from unprocessed urine did not correlate 


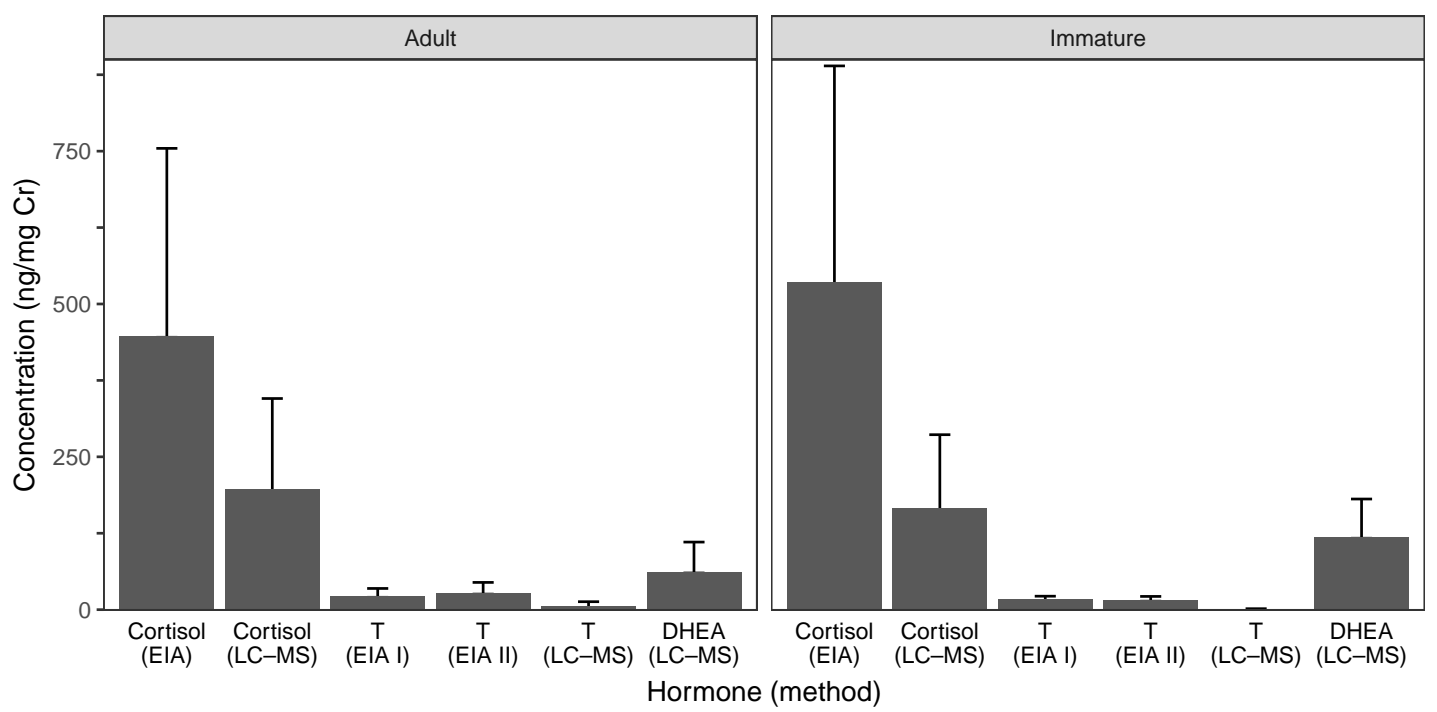

Figure 2.2: Mean urinary hormone levels as measured by LC-MS and EIA. $\mathrm{N}=13$ adult males (except T-EIA II, $\mathrm{N}=10$ ), $\mathrm{N}=8$ immature males. Error bars indicate standard deviation. Cortisol-EIA used unprocessed urine. Testosterone-EIA used hydrolyzed urine. LC-MS used hydrolyzed and solvolyzed urine.

significantly with $\mathrm{uT}_{\mathrm{LC}-\mathrm{MS}}$ in neither adult nor immature males (Table 2.1 and Table 2.2). This suggests that iu $_{\text {EIA }}$ measurements from unprocessed urine do not accurately reflect urinary testosterone levels. Both iuT $\mathrm{T}_{\text {EIA-I }}$ and iuT $\mathrm{T}_{\text {EIA-II }}$ as measured from hydrolyzed urine correlated strongly, positively and significantly with $\mathrm{uT}_{\mathrm{LC}-\mathrm{MS}}$ in adult but not in immature males (Table 2.1 and Table 2.2). This suggests that iu $\mathrm{T}_{\text {EIA }}$ measurements from hydrolyzed urine do accurately reflect urinary testosterone levels in adult but not in immature males.

To determine if the cross-reactivity of the cortisol-antibody with testosterone or DHEA were influencing results, we correlated iuCort EIA $_{\text {with }} \mathrm{uT}_{\mathrm{LC}-\mathrm{MS}}$ and $\mathrm{uDHEA}_{\mathrm{LC}-\mathrm{MS}}$. We found that iuCort ${ }_{\text {EIA }}$ did not correlate significantly with $\mathrm{uT}_{\mathrm{LC}-\mathrm{MS}}$, in neither adult nor immature males (Table 2.1 and Table 2.2). This suggests that cross reaction of the cortisol antibody with urinary testosterone did not have a strong impact on results. However, iuCort EIA did correlate significantly with $\mathrm{uDHEA}_{\mathrm{LC}-\mathrm{MS}}$ in both adult and immature males. To tease apart whether this is a cross-reactivity issue or a true correlation between both adrenal steroids, we also correlated uCort $_{\mathrm{LC}-\mathrm{MS}}$ with $\mathrm{uDHEA} \mathrm{LC}_{\mathrm{MS}}$ and again found a significant positive correlation in both adult and immature males (Bootstrapped Spearman rank correlations: adult: $\mathrm{N}=13$, rho $=0.40$, $95 \% \mathrm{CI}=[0.10,0.68]$; immature: $\mathrm{N}=8$, rho $=0.70,95 \% \mathrm{CI}=[0.24,0.68])$. Taken together, this suggests that the cross-reactivity of the cortisol-antibody with DHEA does not significantly impact iuCort $_{\text {EIA }}$ measurements, but rather that both cortisol and DHEA reflect adrenocortical activity in a similar manner.

To determine if the cross-reactivity of the T-antibodies of EIA I and EIA II with cortisol or DHEA were influencing results, we correlated iuT $_{\text {EIA-I }}$ and iuT $_{\text {EIA-II }}$ with uCort $_{\text {LC-MS }}$ and $\mathrm{uDHEA}_{\mathrm{LC}-\mathrm{MS}}$. Both iuT $\mathrm{T}_{\text {EIA-I }}$ and iuT $\mathrm{T}_{\text {EIA-II }}$ as measured from unprocessed urine correlated 
significantly and positively with $\mathrm{uCort}_{\mathrm{LC}-\mathrm{MS}}$ and $\mathrm{uDHEA}_{\mathrm{LC}-\mathrm{MS}}$ in both adult and immature males (Table 2.1 and Table 2.2). This suggests that cross-reactivity of both T-antibodies with urinary cortisol and DHEA confound iu $\mathrm{T}_{\mathrm{EIA}}$ measurements from unprocessed urine. By contrast, iu $_{\text {EIA-I }}$ as measured from hydrolyzed urine did not correlate significantly with uCort ${ }_{\text {LC-MS }}$ or uDHEA $_{\text {LC-MS }}$ in neither adult nor immature males (Table 2.1 and Table 2.2). This suggests that the cross-reactivity of the T-antibody of EIA I with urinary cortisol or DHEA does not significantly influence iuT $_{\text {EIA-I }}$ measurements in neither adult nor immature males when hydrolyzed urine is used. Similarly, iuT $\mathrm{T}_{\text {EIA-II }}$ as measured from hydrolyzed urine also did not correlate significantly with uCort ${ }_{\mathrm{LC}-\mathrm{MS}}$ or $\mathrm{uDHEA}_{\mathrm{LC}-\mathrm{MS}}$ in adult males (Table 2.1). However, it correlated significantly and positively with uCort ${ }_{\mathrm{LC}-\mathrm{MS}}$ and $\mathrm{uDHEA}_{\mathrm{LC}-\mathrm{MS}}$ in immature males (Table 2.2). This suggests that the cross-reactivity of the T-antibody of EIA II with urinary cortisol or DHEA does not significantly influence iuT $\mathrm{T}_{\text {EIA-II }}$ measurements in adult males but does so in immature males when hydrolyzed urine is used.

Table 2.1: Bootstrapped Spearman rank correlations (rho) between steroids measured by EIA and LC-MS in adult males. Significant correlations (95\% Confidence Interval does not include $0)$ in bold.

\begin{tabular}{llllll}
\hline & & & & \multicolumn{2}{c}{$95 \%$ CI } \\
\cline { 5 - 7 } EIA & LC-MS & N & rho & Lower & Upper \\
\hline \multirow{2}{*}{ Cortisol (unprocessed) } & Cortisol & $\mathbf{1 3}$ & $\mathbf{0 . 9 5}$ & $\mathbf{0 . 9 0}$ & $\mathbf{0 . 9 9}$ \\
& Testosterone & 13 & -0.02 & -0.35 & 0.30 \\
& DHEA & $\mathbf{1 3}$ & $\mathbf{0 . 5 4}$ & $\mathbf{0 . 3 1}$ & $\mathbf{0 . 7 3}$ \\
\hline \multirow{2}{*}{ Testosterone (unprocessed, EIA I) } & Cortisol & $\mathbf{1 3}$ & $\mathbf{0 . 6 9}$ & $\mathbf{0 . 4 9}$ & $\mathbf{0 . 8 3}$ \\
& Testosterone & 13 & 0.12 & -0.30 & 0.50 \\
& DHEA & $\mathbf{1 3}$ & $\mathbf{0 . 7 6}$ & $\mathbf{0 . 5 8}$ & $\mathbf{0 . 9 0}$ \\
\hline \multirow{2}{*}{ Testosterone (unprocessed, EIA II) } & Cortisol & $\mathbf{9}$ & $\mathbf{0 . 3 5}$ & $\mathbf{0 . 1 2}$ & $\mathbf{0 . 5 8}$ \\
& Testosterone & 9 & -0.09 & -0.57 & 0.40 \\
& DHEA & $\mathbf{9}$ & $\mathbf{0 . 7 0}$ & $\mathbf{0 . 5 7}$ & $\mathbf{0 . 8 7}$ \\
\hline \multirow{2}{*}{ Testosterone (hydrolyzed, EIA I) } & Cortisol & 13 & 0.25 & -0.18 & 0.66 \\
& Testosterone & $\mathbf{1 3}$ & $\mathbf{0 . 7 8}$ & $\mathbf{0 . 5 1}$ & $\mathbf{0 . 9 5}$ \\
& DHEA & 13 & 0.23 & -0.10 & 0.52 \\
\hline \multirow{2}{*}{ Testosterone (hydrolyzed, EIA II) } & Cortisol & 10 & -0.03 & -0.37 & 0.47 \\
& Testosterone & $\mathbf{1 0}$ & $\mathbf{0 . 7 6}$ & $\mathbf{0 . 4 5}$ & $\mathbf{0 . 9 5}$ \\
& DHEA & 10 & 0.37 & -0.04 & 0.64 \\
\hline
\end{tabular}

\subsubsection{Comparison of testosterone levels between age classes}

We found that $\mathrm{uT}_{\mathrm{LC}-\mathrm{MS}}$ levels were significantly higher in adult males than immature males (Mann-Whitney $\mathrm{U}$ test: $\mathrm{N}$ adults $=13, \mathrm{~N}$ immatures $=8, \mathrm{U}=6, \mathrm{p}<0.001$, mean adults $=$ $5.7 \pm \mathrm{SD} 7.3 \mathrm{ng} / \mathrm{mg} \mathrm{Cr}$, mean immatures $=0.9 \pm \mathrm{SD} 0.7 \mathrm{ng} / \mathrm{mg} \mathrm{Cr})$. However, this was not the case for iuT $\mathrm{T}_{\text {EIA }}$ measured from hydrolyzed samples (T-EIA I: $\mathrm{N}$ adults $=13, \mathrm{~N}$ immatures $=8, \mathrm{U}=34, \mathrm{p}=0.210$, mean adults $=22.2 \pm \mathrm{SD} 12.5 \mathrm{ng} / \mathrm{mg} \mathrm{Cr}$, mean immatures $=16.9 \pm$ $\mathrm{SD} 5.1 \mathrm{ng} / \mathrm{mg}$ Cr; T-EIA II: $\mathrm{N}$ adults $=10, \mathrm{~N}$ immatures $=8, \mathrm{U}=19, \mathrm{p}=0.068$, mean adults 
Table 2.2: Bootstrapped Spearman rank correlations (rho) between steroids measured by EIA and LC-MS in immature males. Significant correlations (95\% Confidence Interval does not include 0) in bold.

\begin{tabular}{lllllll}
\hline & & & & \multicolumn{2}{c}{$95 \%$ CI } \\
\cline { 5 - 7 } EIA & LC-MS & N & rho & Lower & Upper \\
\hline Cortisol (unprocessed) & Cortisol & $\mathbf{8}$ & $\mathbf{0 . 8 8}$ & $\mathbf{0 . 7 1}$ & $\mathbf{1 . 0 0}$ \\
& Testosterone & 8 & -0.03 & -0.52 & 0.36 \\
& DHEA & $\mathbf{8}$ & $\mathbf{0 . 9 0}$ & $\mathbf{0 . 6 4}$ & $\mathbf{1 . 0 0}$ \\
\hline \multirow{2}{*}{ Testosterone (unprocessed, EIA I) } & Cortisol & $\mathbf{8}$ & $\mathbf{0 . 7 3}$ & $\mathbf{0 . 4 3}$ & $\mathbf{0 . 9 3}$ \\
& Testosterone & 8 & 0.22 & -0.33 & 0.64 \\
& DHEA & $\mathbf{8}$ & $\mathbf{0 . 8 3}$ & $\mathbf{0 . 6 7}$ & $\mathbf{0 . 9 5}$ \\
\hline \multirow{2}{*}{ Testosterone (unprocessed, EIA II) } & Cortisol & $\mathbf{7}$ & $\mathbf{0 . 8 7}$ & $\mathbf{0 . 7 1}$ & $\mathbf{1 . 0 0}$ \\
& Testosterone & 7 & -0.12 & -0.68 & 0.39 \\
& DHEA & $\mathbf{7}$ & $\mathbf{0 . 8 5}$ & $\mathbf{0 . 6 4}$ & $\mathbf{0 . 9 6}$ \\
\hline \multirow{2}{*}{ Testosterone (hydrolyzed, EIA I) } & Cortisol & 8 & 0.52 & -0.14 & 0.93 \\
& Testosterone & 8 & 0.36 & -0.36 & 0.88 \\
& DHEA & 8 & 0.36 & -0.26 & 0.83 \\
\hline \multirow{2}{*}{ Testosterone (hydrolyzed, EIA II) } & Cortisol & $\mathbf{8}$ & $\mathbf{0 . 8 3}$ & $\mathbf{0 . 6 2}$ & $\mathbf{0 . 9 8}$ \\
& Testosterone & 8 & -0.04 & -0.62 & 0.60 \\
& DHEA & $\mathbf{8}$ & $\mathbf{0 . 9 0}$ & $\mathbf{0 . 8 3}$ & $\mathbf{0 . 9 3}$ \\
\hline
\end{tabular}

$=27.5 \pm \mathrm{SD} 17.1 \mathrm{ng} / \mathrm{mg} \mathrm{Cr}$, mean immatures $=15.9 \pm \mathrm{SD} 5.8 \mathrm{ng} / \mathrm{mg} \mathrm{Cr})$.

\subsection{Discussion}

In this study, we validated the use of cortisol and testosterone EIAs in the urine of adult and immature male Barbary macaques by comparing results to those from LC-MS measurements. We found that iuCort EIA measurements from unprocessed urine correlated strongly with uCort $_{\mathrm{LC}-\mathrm{MS}}$ in both adult and immature males. This indicates that the direct measurement of cortisol by EIA is a reliable method for assessing adrenocortical activity in adult and immature male Barbary macaques. By contrast, iuT $\mathrm{T}_{\mathrm{EIA}}$ measurements only correlated with $\mathrm{uT}_{\mathrm{LC}-\mathrm{MS}}$ if steroids in the urine were enzymatically hydrolyzed prior to analysis and only in adult males. In immature males, even iu $\mathrm{T}_{\mathrm{EIA}}$ measurements from hydrolyzed urine did not correlate significantly with $\mathrm{uT}_{\mathrm{LC}-\mathrm{MS}}$. This lack of correlation is likely due to cross-reactivity of the testosterone antibody with other adrenal steroids such as cortisol and DHEA, which are present at much higher concentrations relative to testosterone in immature males. Furthermore, $\mathrm{uT}_{\mathrm{LC}-\mathrm{MS}}$ - but not iuT $\mathrm{T}_{\mathrm{EIA}}$ - levels were significantly higher in adult than immature males, biologically validating the measurement of testosterone by LC-MS but not by EIA. Taken together, our results suggest that the testosterone EIAs are suitable to assess gonadal endocrine activity from urine analysis in adult but not immature male Barbary macaques, and only if a hydrolysis is conducted prior to analysis. 


\subsubsection{Pattern of conjugation of cortisol and testosterone}

Both cortisol and testosterone were primarily excreted as glucuronide conjugates and only a small proportion was excreted as sulfates. Cortisol was also excreted in substantial quantities in its free (i.e. unconjugated) form, but testosterone was not. This is consistent with other studies that found that these steroids are primarily excreted in conjugated form, and mainly as glucuronides, in the urine of primate (Bahr et al., 2000; Möhle et al., 2002; Ziegler et al., 2000) and non-primate species (Teskey-Gerstl et al., 2000).

\subsubsection{Steroid levels in urine}

We found that both iuCort ${ }_{\text {EIA }}$ and iuT $\mathrm{T}_{\text {EIA }}$ levels were significantly higher than uCort $_{\text {LC-MS }}$ and $\mathrm{uT}_{\mathrm{LC}-\mathrm{MS}}$, respectively, a result consistent with findings from other studies that have compared levels of these two steroids in males, as measured from immunoassays and LC-MS (Baid et al., 2007; Hsing et al., 2007; Preis et al., 2011; Welker et al., 2016). Indeed, these "inflation" in absolute hormone concentration as measured by EIA is not surprising given that, in contrast to LC-MS measurements, immunological hormone measurements are generally non-specific as they are influenced by cross-reactivity characteristics of the antibody used. In line with this argument, the difference between EIA and LC-MS measurements is larger in urine samples than in blood samples (Preis et al., 2011). As previously mentioned, in blood, steroids primarily circulate in their native, unconjugated form at much higher proportions than in urine so the impact of cross-reactivity of the antibody is lower in blood samples than in urine (Preis et al., 2011; Venturelli et al., 1995). It is important to note that the cross-reactivity of the antibody in immunoassays are not necessarily disadvantageous. As long as the cross-reacting metabolites originate from the same parent hormone, this may actually be advantageous as such so-called group-specific assays may have a higher sensitivity to detect a biologically meaningful change in hormone levels (e.g. Heistermann et al., 2006; Shutt et al., 2012).

\subsubsection{Correlations between urinary cortisol measured by EIA and urinary steroids measured by LC-MS}

We compared cortisol EIA and LC-MS results to determine if the EIA measurements truly reflect urinary cortisol levels and to determine if cross-reactivities with testosterone or DHEA were confounding results. We found that iuCort ${ }_{\text {EIA }}$ measured from unprocessed urine correlated strongly and significantly with $\mathrm{uCort}_{\mathrm{LC}-\mathrm{MS}}$ for both adult and immature males. This strong correlation suggests that the iuCort EIA measurements accurately reflect urinary cortisol levels and was achieved without the need to perform deconjugation steps in the urine samples prior to analysis by EIA. It is likely that cortisol was excreted in sufficiently high quantities in its unconjugated form to be measured by the EIA, despite the fact the most of it was excreted as glucuronides. Alternatively, but not mutually exclusive, our cortisol antibody may potentially 
also cross-react with and measure cortisol in its conjugated forms. The markedly higher absolute levels of cortisol measured by EIA compared to LC-MS (see above) would be in line with such an assumption. Since the deconjugation step is time-consuming and costly, it is worthwhile to check if this analytical step can be avoided prior to any urinary hormone analysis (like in this study) to still produce reliable results.

Cross-reactivities of our cortisol-antibody with urinary testosterone and DHEA were negligible. We found that iuCort ${ }_{\text {EIA }}$ did not correlate significantly with $\mathrm{uT}_{\mathrm{LC}-\mathrm{MS}}$ in neither adult nor immature males but did have a significant positive correlation with $\mathrm{uDHEA}_{\mathrm{LC}-\mathrm{MS}}$ in both adult and immature males. Rather than this correlation being caused by a cross-reactivity problem, our results indicate that the correlation was caused by simultaneous secretion as uCort $_{\mathrm{LC}-\mathrm{MS}}$ and $\mathrm{uDHEA}_{\mathrm{LC}-\mathrm{MS}}$ were also significantly correlated. The strength of the correlation between uCort $_{\mathrm{LC}-\mathrm{MS}}$ (or also iuCort $\mathrm{EIA}_{\mathrm{A}}$ ) and $\mathrm{uDHEA}_{\mathrm{LC}-\mathrm{MS}}$ was stronger in immature than adult males, possibly indicating a stronger interrelationship between these two adrenal steroids in immature compared to adult males.

Generally, urinary cortisol (or corticosterone) analysis is often used as a measure of adrenocortical activity in various species of mammals (Aronsen et al., 2015; Brown et al., 2010; McCallister et al., 2004; McLeod et al., 1996; Schmid et al., 2001; Touma et al., 2003). In this respect, the cortisol-EIA tested in this study has been shown to successfully pick up the urinary cortisol response to an ACTH challenge in African elephants (Ganswindt et al., 2003). It has also been demonstrated to measure urinary cortisol levels in other nonhuman primates (Bahr et al., 2000), indicating its suitability to non-invasively assess cortisol secretion in various mammalian species. Therefore, we envisage that the measure of urinary cortisol in unprocessed urine as described here is likely suitable for tracking adrenocortical activity in male Barbary macaques, although, to support these results, an accompanying physiological or biological validation would be desirable.

\subsubsection{Correlations between urinary testosterone measured by EIA and urinary steroids measured by LC-MS}

We compared two testosterone EIAs with LC-MS results to determine if the EIA measurements truly reflect urinary testosterone levels and to determine if cross-reactivity of the antibodies with steroids of adrenal origin, such as cortisol or DHEA, were potentially confounding results. Both iu $\mathrm{T}_{\text {EIA-I }}$ and iu $\mathrm{T}_{\text {EIA-II }}$ measurements from unprocessed urine did not correlate significantly with $\mathrm{uT}_{\mathrm{LC}-\mathrm{MS}}$ in neither adult nor immature males. Since $>80 \%$ of testosterone was excreted as glucuronide conjugate, we assessed whether enzymatic hydrolysis would result in stronger correlations between our T-EIA and LC-MS measurements. Indeed, this extra analytical step largely improved the correlations between the two data sets so that both iu $\mathrm{T}_{\text {EIA-I }}$ and iuT $\mathrm{T}_{\text {EIA-II }}$ measurements from hydrolyzed urine now correlated strongly with $\mathrm{uT}_{\mathrm{LC}-\mathrm{MS}}$ in adult males but not immature males. It is likely that cross-reactivity of the testosterone antibodies with cortisol and DHEA, and probably also other adrenal steroids, are confounding the results generated from 
unprocessed urine in adult and immature males and from hydrolyzed urine in immature males. This is supported by the fact that $\mathrm{uCort}_{\mathrm{LC}-\mathrm{MS}}$ and $\mathrm{uDHEA}_{\mathrm{LC}-\mathrm{MS}}$ were both positively correlated with iuT $\mathrm{T}_{\text {EIA-I }}$ and $\mathrm{iuT}_{\text {EIA-II }}$ when measured from unprocessed urine in both age classes, and with iuT $_{\text {EIA-II }}$ - but not iuT $\mathrm{T}_{\text {EIA-I }}$ - when measured from hydrolyzed urine in immature males.

Obviously, the cross-reactivities of the antibodies in the T-EIAs with other metabolites are constant and do not change whether being used with unprocessed or hydrolyzed urine. The observed changes in the strength of correlations with LC-MS measurements are caused by the differences in the ratio of unconjugated testosterone to cross-reacting adrenal steroids. For instance, adult males have uCort ${ }_{\mathrm{LC}-\mathrm{MS}}$ and $\mathrm{uDHEA}_{\mathrm{LC}-\mathrm{MS}}$ concentrations approximately 35 and 11 times higher than $\mathrm{uT}_{\mathrm{LC}-\mathrm{MS}}$, respectively (Fig. 2.2). By contrast, immature males have approximately 186 and 134 times higher uCort ${ }_{\mathrm{LC}-\mathrm{MS}}$ and $\mathrm{uDHEA}_{\mathrm{LC}-\mathrm{MS}}$ levels than $\mathrm{uT}_{\mathrm{LC}-\mathrm{MS}}$, respectively (Fig. 2.2). In both T-EIAs, the cross-reactivity of the antibody with cortisol and DHEA is $<0.1 \%$ (Kersey et al., 2010; Palme \& Möstl, 1994). Thus, $<4 \%$ and $<1 \%$ of the EIA results from hydrolyzed urine can be attributed to cross-reactivity with cortisol and DHEA, respectively, in adult males. This relatively low level of co-measurement of the two adrenal hormones does not obviously confound the T-EIA measurement in adult males as evidenced by the strong positive correlations that both iu $\mathrm{T}_{\text {EIA-I }}$ and iu $\mathrm{T}_{\text {EIA-II }}$ have with $\mathrm{uT}_{\mathrm{LC}-\mathrm{MS}}$ when measured from hydrolyzed urine samples. However, in immature males the picture looks different. Around $19 \%$ and $13 \%$ of the T-EIA results from hydrolyzed urine can be attributed to cross-reactivity with cortisol and DHEA, respectively, a proportion sufficiently large enough to confound testosterone levels measured by EIA in hydrolyzed urine of immature males. Since the vast majority of testosterone was excreted as testosterone glucuronide in male Barbary macaque urine (Fig. 2.1), the absolute concentration of unconjugated testosterone in unprocessed urine was apparently low enough that even in adult males, cross-reactivity with other compounds became a problem. Similar to our results, testosterone immunoassays that have been validated for use in males did not produce reliable results in females, likely due to lower concentration of testosterone and higher proportion of cross-reacting adrenal steroids in females (Goymann, 2005; Preis et al., 2011).

Importantly, cortisol and DHEA are only two examples of hormones coming from a non-gonadal, i.e. the adrenal pathway, whose cross-reaction with the testosterone antibody, potentially affects the measurements of urinary testosterone with the tested T-EIAs. However, there are many other candidate urinary steroid metabolites with a similar potential. For example, one study in chimpanzees found that just among the limited number of potential glucocorticoid and androgen metabolites measured, there were 10 steroids present in the urine of males at higher concentrations than testosterone (Hauser et al., 2008a). This potentially leads to a situation where the overall sum of cross-reactivities with all these metabolites, even if the cross-reactivity with each one is very small, will have a much bigger effect than what we describe in this study. This danger is more and more prominent the lower the ratio of the target hormone to confounding metabolites. Concordantly, cortisol, as the most abundant steroid measured in this study, is the least affected by this problem and produced the strongest correlations between EIA and LC-MS measurements. As we show in this study, one approach to mitigate the impact of 
antibody cross-reactivity in urine samples is to increase the ratio of the target hormone through deconjugation.

\subsubsection{Comparison of testosterone levels between age classes}

To assess whether our EIA and LC-MS measurements were able to capture biologically meaningful differences in hormone levels, we compared testosterone and DHEA levels of adult and immature males. As predicted, $\mathrm{uT}_{\mathrm{LC}-\mathrm{MS}}$ was significantly higher in adult males compared to immature males, biologically validating the measurement of testosterone by LC-MS in male Barbary macaques. However, there was no significant difference in iuT $\mathrm{T}_{\text {EIA }}$ levels between age classes as measured by either EIAs from hydrolyzed urine. As discussed above, this is likely because in immature males, a substantial co-measurement of adrenal steroids in both T-EIAs increases the concentrations of immunoreactive testosterone and thereby obscures the existing difference in "real" testosterone levels between the two age classes. Similarly to our results, others have found that a testosterone antibody cross-reacting with DHEA metabolites has masked the predicted difference in testosterone levels between adult males and females or castrated males (Goymann, 2005; Möhle et al., 2002). Taken together, our results suggest that measuring testosterone with either EIA from hydrolyzed urine is suitable to assess gonadal endocrine activity in adult but not immature male Barbary macaques. This study highlights that even when a biological validation of a testosterone EIA fails, the tested EIA might still be suitable for specific age and sex classes. At the same time, just because an EIA reliably measures testosterone in one age sex class, e.g. adult males, does not necessarily make it suitable for all other age sex classes. By not only comparing EIA measurements across age sex classes but also correlating EIA with LC-MS measurements we were able to determine in which age class results were reliable and in which they were unreliable.

\subsection{Conclusions}

Combining LC-MS and EIA approaches, we identified two main issues to consider when measuring steroid hormones from urine samples using immunoassays: pattern of conjugation and cross-reactivities of the antibody. Both the pattern of conjugation and cross-reactivity are most likely to confound results when the absolute concentration of the target hormone is low relative to other cross-reacting metabolites. As cortisol was excreted in sufficiently high concentrations in its native form in our study subjects, results from EIA and LC-MS were strongly correlated in both adult and immature males, making deconjugation steps unnecessary. Deconjugation of urinary steroid hormones can also be avoided if the antibodies used have a substantial cross-reactivity with the conjugated forms (Al-Dujaili, 2006; Eastman et al., 1984; Heistermann \& Hodges, 1995). Testosterone was excreted in urine in much lower concentrations than cortisol and a higher proportion of it was conjugated, primarily as glucuronides, necessitating hydrolysis of steroids to ensure that a strong correlation of 
EIA compared to LC-MS results was obtained in adult male samples. However, even after hydrolysis, EIA measurements of testosterone in immature male urine samples were still not reliable, most likely due to cross-reactivity with adrenal steroids, which occur at much higher concentrations than testosterone in immature males. Thus, to generate data on gonadal endocrine activity in immature Barbary macaques, LC-MS analysis may be the most reliable approach. We envisage that immunological assays may potentially also be applied successfully on immature samples if highly specific testosterone (metabolite) antibodies with almost no cross-reactivities with steroids of adrenal origin are available. However, given the generally low production of testosterone in immature males, it is possible that most immunoreactive androgens in the urine of immatures are breakdown products of androgens of adrenal origin. To confirm that urinary measurements of androgens in immature males (and possibly also females), whether done with LC-MS or EIA, are indeed stemming from serum testosterone of gonadal origin, requires a comparison between serum and urine levels. Measurement of fecal testosterone metabolites may provide an alternative to urinary measurements (Rincon et al., 2017), although similar validation work as described here for urine analysis is necessary to firmly confirm this. Taken together, both testosterone EIAs can be used in studies assessing gonadal activity in adult but not immature male Barbary macaques. Even within the same sex, age-related changes in androgen production can cause the same immunoassay to be reliable in one but not another age class. Given differences between steroids in terms of conjugation patterns and absolute levels, preparatory steps in terms of deconjugation and extraction will vary between different EIAs and hormones.

\section{Acknowledgements}

We thank Ellen Merz and Roland Hilgartner for permission to conduct the study. We thank Lauren Cassidy, Tatjana Kaufmann and Lilah Sciaky for help with urine sample collection. We are grateful to Andrea Heistermann and Miriam Polten for support with the EIA measurements and general assistance in the lab, and to Roisin Murtagh and Vera Schmeling for help with the LC-MS analysis. The study benefitted from discussions within the DFG-research group "Sociality and health in primates" (FOR 2136). This research was funded by the Deutsche Forschungsgemeinschaft (DFG, German Research Foundation) - Project number 254142454 / GRK 2070. Consumables for the LC-MS analysis were funded by the Max Planck Society while all reagents for the EIA analyses were funded by the German Primate Center. 


\section{Chapter 3}

\section{Oxytocin increases after affiliative interactions in male Barbary macaques}

Alan V. Rincon ${ }^{\mathrm{a}, \mathrm{b}}$, Tobias Deschner ${ }^{\mathrm{c}}$, Oliver Schülke $\mathrm{e}^{\mathrm{a}, \mathrm{b}, \mathrm{d}}$, Julia Ostner ${ }^{\mathrm{a}, \mathrm{b}, \mathrm{d}}$

${ }^{a}$ Department of Behavioral Ecology, Johann-Friedrich-Blumenbach Institute for Zoology and Anthropology, University of Goettingen, Goettingen, Germany

${ }^{b}$ Leibniz ScienceCampus Primate Cognition, Goettingen, Germany

${ }^{c}$ Department of Primatology, Max Planck Institute for Evolutionary Anthropology, Leipzig, Germany

${ }^{d}$ Research Group Social Evolution in Primates, German Primate Center, Leibniz Institute for Primate Research, Goettingen, Germany

Hormones and Behavior, 119, 104661 (2020). https://doi.org/10.1016/j.yhbeh.2019.104661. 


\section{Abstract}

Mammals living in stable social groups often mitigate the costs of group living through the formation of social bonds and cooperative relationships. The neuropeptide hormone oxytocin (OT) is proposed to promote both bonding and cooperation although only a limited number of studies have investigated this under natural conditions. Our aim was to assess the role of OT in bonding and cooperation in male Barbary macaques (Macaca sylvanus). First, we tested for an effect of affiliation - grooming and triadic male-infant-male interactions - with bond and non-bond partners on urinary OT levels. Second, we tested whether grooming interactions (and thus increased OT levels) increase a male's general propensity to cooperate in polyadic conflicts. We collected $>4000$ hours of behavioral data on 14 adult males and measured OT levels from 139 urine samples collected after affiliation and non-social control periods. Urinary OT levels were higher after grooming with any partner. By contrast, OT levels after male-infant-male interactions with any partner or with bond partners were not different from controls but were higher after interactions with non-bond partners. Previous grooming did not increase the likelihood of males to support others in conflicts. Collectively, our results support research indicating that OT is involved in the regulation of adult affiliative relationships. However, our male-infant-male interaction results contradict previous studies suggesting that it is affiliation with bond rather than non-bond partners that trigger the release of OT. Alternatively, OT levels were elevated prior to male-infant-male interactions thus facilitating interaction between non-bond partners. The lack of an association of grooming and subsequent support speaks against an OT linked increase in the general propensity to cooperate.

Keywords: oxytocin, social bonds, cooperation, grooming, male-infant-male interaction, Barbary macaque 


\section{$3.1 \quad$ Introduction}

For mammals living in stable social groups, investing in strong social bonds can provide individuals with adaptive benefits (Ostner \& Schülke, 2018) such as increased reproductive success (Cameron et al., 2009; Frère et al., 2010; Schülke et al., 2010; Strauss \& Holekamp, 2019; Weidt et al., 2008) and increased survival (Archie et al., 2014; Giles et al., 2005; Silk et al., 2010). Social bonds can be defined as strong, equitable and enduring affiliative relationships (Silk, 2002) and may promote cooperative behavior (Schülke et al., 2010; Smith et al., 2011; Weidt et al., 2008; Young et al., 2014b) and buffer physiological impacts of stress (Cheney \& Seyfarth, 2009; Wittig et al., 2016; Young et al., 2014a).

One hormone implicated in the formation and maintenance of social bonds is the highly conserved neuropeptide oxytocin (OT). OT plays a role in promoting maternal behavior (Finkenwirth et al., 2016; Ross \& Young, 2009) and ultimately a partner specific attachment between mother and offspring (Ross \& Young, 2009). The oxytocinergic system is thought to have been co-opted from its ancestral function of mother-offspring attachment to also promote social bonds between adults (Numan \& Young, 2016; Ziegler \& Crockford, 2017). This has been best demonstrated in the context of pair bonds where OT helps regulate a social preference for a particular mating partner (French et al., 2018; Ross \& Young, 2009). Oxytocin may also regulate affiliative relationships more broadly outside the pair-bond and in non-reproductive contexts. In support of this, OT is released after positive, non-sexual, social interactions (chimpanzees, Pan troglodytes: Crockford et al., 2013; Preis et al., 2018; Wittig et al., 2014; tufted capuchins, Sapajus apella: Benítez et al., 2018; dogs, Canis familiaris: Romero et al., 2014). Because OT interacts with the reward system (Dölen et al., 2013; Love, 2014; Skuse \& Gallagher, 2009), OT release potentially stimulates a 'feel good' sensation after positive social interactions. These sensations may be part of the mechanism by which social bonds are maintained (e.g. via emotional bookkeeping: Schino \& Aureli, 2009).

OT release is partner specific, at least in some studies. In chimpanzees, urinary OT levels are increased above control levels after grooming with a bonded partner, but not after the same interaction with a non-bonded partner (Crockford et al., 2013). Similarly, in cooperatively breeding marmosets (Callithrix jacchus), strongly bonded family members show synchronous fluctuations in baseline urinary OT levels whereas partners with weaker relationships do not, suggesting that affiliation with bond partners influences OT levels more than affiliation with non-bond partners (Finkenwirth et al., 2015). Other studies in bonobos (Pan paniscus) and two different populations of chimpanzees, however, suggest that OT is released independently of affiliative relationship strength (Moscovice et al., 2019; Preis et al., 2018; Wittig et al., 2014) or that the impact of relationship strength on OT secretion depends on the type of interaction (Wittig et al., 2014).

In addition to social bonding, oxytocin plays a key role in promoting coordination and cooperative behaviors under certain contexts. In humans, intranasal administration of OT increased cooperation in economic games when participants had prior contact but not when they 
were anonymous (Declerck et al., 2010), and similarly in in-group but not out-group conditions (De Dreu et al., 2010; Ten Velden et al., 2017). Performance on a cooperative task was also improved by OT administration, suggestive of OT's role in the facilitation of coordination of behavior (Arueti et al., 2013). These findings in humans are paralleled in chimpanzees where urinary OT levels were elevated during coordinated behaviors such as territorial border patrols (Samuni et al., 2017) and cooperative hunting (Samuni et al., 2018a) compared to controls. Furthermore, the highest levels of urinary OT in chimpanzees occurred during inter-group encounters, which involve joint aggression against out-group members (Samuni et al., 2017). Depending on context, OT appears to facilitate participation in polyadic aggression through increased coordination and in-group favoritism in humans and chimpanzees. The generality of these patterns beyond these taxa remains to be tested.

We aimed to investigate the role of oxytocin in the maintenance of social bonds and cooperation in male macaques. Macaque males of several species form strong, equitable and stable social bonds with other males (Kalbitz et al., 2016; Young et al., 2014b) which are predictive of cooperation via coalition formation (Berghänel et al., 2011a; Schülke et al., 2010; Young et al., 2014b). Coalitions serve to increase or maintain male dominance rank (Young et al., 2014c), and increase mating success (Küster \& Paul, 1992; Young et al., 2013a) or reproductive success (Schülke et al., 2010). In addition to male-male bonds, macaques may also from strong male-female bonds (Haunhorst et al., 2016; Massen \& Sterck, 2013). Males frequently support females in agonistic conflicts (Haunhorst et al., 2017; Kulik et al., 2012; Small, 1990), and similarly to male-male relations, the probability to support is predicted by social bond strength (Haunhorst et al., 2017; Kulik et al., 2012).

A behavioral pattern proposed to enhance male social bonding are triadic male-infant-male interactions, which are characteristic yet not exclusive to Barbary macaques (Deag, 1980; Paul et al., 1996). These interactions are ritualistic in nature and involve two males sitting in body contact holding an infant in between them while teeth-chattering and often inspecting the infant's genitals (Deag, 1980; Deag \& Crook, 1971). This behavior most commonly involves newborn infants, although yearlings and two-year-olds are also sometimes involved (Paul et al., 1996). Triadic male-infant-male interactions have been proposed to be used as a tool to enhance male-male social bonds (Henkel et al., 2010; Kalbitz et al., 2017; Paul et al., 1996) and been shown to predict coalition formation in the mating season (Berghänel et al., 2011a). Alternatively, though not mutually exclusive, male-infant-male interactions may be used as a form of 'agonistic buffering' (Deag, 1980; Deag \& Crook, 1971; Paul et al., 1996). Thus, triadic male-infant-male interaction is a behavior with qualities similar to others that induce the release of OT.

Similar to previous studies in chimpanzees and tufted capuchins (Benítez et al., 2018; Crockford et al., 2013), we tested in Barbary macaques whether urinary OT levels were influenced by grooming interactions and - given its functional relevance in Barbary macaques - also by male-infant-male interactions. We predicted that urinary OT levels would be higher after affiliative interactions (i.e., grooming, male-infant-male interactions) compared to a control 
period without any social interactions (prediction 1). We additionally tested whether the release of OT was partner-specific (i.e., bond partners vs. non-bond partners). If OT release is partner-specific, we predicted that it will be higher after affiliations with bond partners than with non-bond partners (prediction 2). A secondary aim was to test whether OT would generally increase the propensity to cooperate in within-group polyadic agonistic conflicts. To do this under natural conditions, we first determined which affiliative behaviors are positively related to urinary OT levels (as part of the first aim), to use the occurrence of this interaction as a proxy for elevated OT levels in the subject. We consequently predicted, that the probability to cooperate, i.e. to support another individual in an agonistic conflict, would be higher after an affiliative interaction (prediction 3).

\subsection{Materials and methods}

\subsubsection{Study site and animals}

Study subjects belonged to one of three groups of Barbary macaques living together in 14.5 ha. of enclosed forest at Affenberg Salem, Germany (de Turckheim \& Merz, 1984). Monkeys were provisioned once daily with fruits, vegetables, grains and had ad libitum access to water and monkey chow. Data collection took place from 31 March to 17 December 2016, including one non-mating season (31 March to 26 October) and one mating season (27 October to 17 December). The start of the mating season was defined by the first observed ejaculatory copulation. The study group (group C) consisted of 13-14 adult males (one male died during the study period), 20 adult females, 2 large subadult males, 8 immature males, 10 immature females and 1 newborn infant male. All members of the group were individually recognized by observers.

\subsubsection{Behavioral data collection}

Behavioral data were collected from 14 adult males using continuous focal animal sampling (Martin \& Bateson, 2007) during individual full-day focal animal follows, in which the occurrence and partners of all social interactions were recorded (Total $=4252$ hours, $304 \pm$ SD 39 hours per individual). Inter-observer reliability was checked and confirmed periodically throughout the study period.

\subsubsection{Assessing dyadic bond strength}

To assess affiliative relationship strength, we calculated the dyadic Composite Sociality Index (CSI; Silk et al., 2010), with slight modifications as described in Haunhorst et al. (2016). This 
index ranges from 0 to infinity and has a mean value of 1 , where higher CSI scores indicate a stronger relationship. To calculate the CSI, we chose seven significantly correlated affiliative behavioral variables: duration and count of close proximity $(\leq 1.5$ meters) without aggression, duration and count of body contact, duration and count of grooming and count of triadic male-infant-male interactions. Both duration and count of behaviors were corrected for the total observation time of the dyad. Male Barbary macaques affiliate with females much more frequently than with other males (mean \pm SD behavior seconds per observation hour per sex of dyad: proximity, male-male dyads $=69 \pm 57$; proximity, male-female dyads $=372 \pm 121$; body contact, male-male dyads $=36 \pm 38$; body contact, male-female dyads $=247 \pm 71$; grooming, male-male dyads $=22 \pm 20$; grooming, male-female dyads $=201 \pm 55)$. If we had included both male-male and male-female dyads into a single CSI scores this would lower the CSI scores for male-male dyads. As a result, we would potentially misclassify some male-male dyads as non-bond when in fact they are bond partners, given that male-male bonds are meaningful and have adaptive significance (see introduction). Therefore, we constructed separate CSI scores for male-male and male-female dyads. The two large subadult males in our study group were included in the calculation of the male-male CSI scores because they supported other adult males in agonistic conflicts. Furthermore, we calculated separate CSI scores for the non-mating and mating seasons as affiliation patterns may change across seasons. Out of the seven affiliative behavior conditions, we only included them in the CSI calculation if their mean frequency of occurrence per dyad in each period was $>2$ to avoid rare behaviors disproportionately affecting the CSI scores. We defined bond partners as those dyads with a CSI score $>1$ (above the group mean).

\subsubsection{Urine sample collection}

Urine samples were collected opportunistically from individuals during focal follows. When monkeys were seen to urinate, the urine was caught with a plastic bag when possible or collected from leaves, branches, rocks or the ground by using a disposable pipette or salivette (Salivette Cortisol, Sarstedt, Nümbrecht, Germany). The use of salivettes to collect urine has recently been validated and successfully applied to urine samples from free-ranging macaques (Danish et al., 2015; Müller et al., 2017). Urine samples contaminated with feces, blood or urine from other individuals were not collected. Urine samples collected by pipette were transferred to $2 \mathrm{ml}$ cryotubes. Both samples stored in cryotubes and salivettes were kept in a thermos flask filled with ice while in the field. At the end of the day, urine was recovered from the salivettes by centrifugation for $5 \mathrm{~min}$ at $1500 \mathrm{rpm}$ using an electric centrifuge and also transferred to $2 \mathrm{ml}$ cryotubes. Samples were split into two aliquots (100 to $2000 \mathrm{\mu l}$ each). One aliquot was used for analysis of creatinine. In the second aliquot, $0.5 \mathrm{~N}$ phosphoric acid were added to urine at a ratio of 1:10 acid to urine to prevent the breakdown of OT in the sample (Reyes et al., 2014; Ziegler, 2018). All samples were then stored in a freezer at $-20^{\circ} \mathrm{C}$. When data collection was complete, samples were transported in containers with dry ice to the lab and stored once again at $-20^{\circ} \mathrm{C}$. 
Urine samples were collected from all 14 adult males ( 7 to 25 years old) of the study group. We presumed a clearance window of 15 to $60 \mathrm{~min}$ for excretion of OT in urine, as done in previous studies investigating urinary OT levels in other non-human primates which show biologically relevant changes in behavior during this window (Benítez et al., 2018; Crockford et al., 2013; Samuni et al., 2017). Studies in humans and marmosets have demonstrated elevated OT levels in urine 30 to $60 \mathrm{~min}$ after administration of radio-labelled hormone (humans: Amico et al., 1987; marmosets: Seltzer \& Ziegler, 2007). Exogenous administration of OT in tufted capuchin monkeys also caused elevated urinary OT levels 15 to $60 \mathrm{~min}$ after administration (Benítez et al., 2018). Prior to analysis, urine samples were assigned to different behavioral conditions depending on whether at least one grooming (total time $\geq 60 \mathrm{sec}$ ), triadic male-infant-male interaction or no social interactions occurred in the $45 \mathrm{~min}$ clearance window. As we were interested in the role of OT in bonding in a non-sexual context, we only considered samples collected during the non-mating season for analysis. Furthermore, samples were excluded from analysis if any ejaculatory copulations, play or coalitions co-occurred in the clearance window because these behaviors could potentially influence OT levels and confound results. For the oldest adult male in the group, we were not able to collect any urine samples where our target behaviors occurred within the OT clearance window and therefore this male was not included in analyses for predictions 1 and 2. This left us with 76 non-social (control) samples (mean = 5.8 , range $=2-11$ per individual) and 63 samples where at least one affiliation occurred (test samples: mean $=4.8$, range $=1-9$ per individual) from 13 adult males.

\subsubsection{Extraction and hormone analysis}

The extraction and analysis of OT followed a protocol described in detail in Samuni et al. (2017). Briefly, urine samples were thawed and kept cool using an Iso-rack ( $0^{\circ} \mathrm{C}$; Eppendorf). Then samples were centrifuged for $1 \mathrm{~min}$ at $1500 \mathrm{rpm}$ at $4^{\circ} \mathrm{C}$. Solid-phase extraction cartridges (Chromabond HR-X, 30mg, $1 \mathrm{ml}$, Macherey-Nagel, Dueren, Germany) were conditioned with 1 $\mathrm{ml} \mathrm{MeOH}$ followed by $1 \mathrm{ml}$ distilled HPLC-water. Cartridges were then filled with up to $1 \mathrm{ml}$ dilution buffer (water, $0.1 \%$ TFA) and 20 to $100 \mu$ l of urine. Diluted urine was allowed to run through the cartridge. Then, the cartridge was washed with $1 \mathrm{ml}$ washing solution $(10 \% \mathrm{ACN}$, $1 \%$ TFA) and dried using a vacuum. Hormones were eluted using $1 \mathrm{ml}$ ACN $80 \%$ into clean test tubes. Elutes were evaporated at $50^{\circ} \mathrm{C}$ with pressurized air. Then $300 \mu \mathrm{l} \mathrm{EtOH} 100 \%$ was added to each test tube and shaken gently. Test tubes were allowed to sit for 1 hour at $4^{\circ} \mathrm{C}$ to precipitate proteins before being evaporated again at $50^{\circ} \mathrm{C}$. Samples were then reconstituted with $250 \mu \mathrm{l}$ assay buffer from a commercially available enzyme immunoassay kit (Assay Designs; 901-153A-0001), and vortexed gently for $10 \mathrm{sec}$ by hand. Extracts were then transferred to 1.5 ml labeled eppendorf tubes, and vortexed for $1 \mathrm{~min}$ at 10,000 rpm. Extracts were then kept cool on ice while preparing the assay. The assay was then performed according to instructions provided by the manufacturer.

To determine the efficiency of the extraction protocol, we created 5 pools of Barbary macaque urine samples. Before extraction, $75 \mu \mathrm{l}$ of each pooled sample were spiked with $75 \mu \mathrm{l}$ of an OT 
standard $(1500 \mathrm{pg} / \mathrm{ml})$. We used the values from the spiked and unspiked samples to calculate percent recovery for extraction efficiency and assay accuracy following the formula given in Behringer et al. (2012). Mean extraction was $81.0 \%$ (range: $68.7-92.7 \%, \mathrm{SD}=10.2, \mathrm{~N}=5$ ). We investigated matrix effects that could potentially interfere with the assay system by testing for parallelism. Out of a pool sample, we took $3 \mathrm{ml}$ of urine and extracted them according to our extraction protocol. Of the resulting $500 \mu \mathrm{l}$ of extract, $250 \mathrm{\mu l}$ were taken and serially diluted. Another $1 \mathrm{ml}$ of the urine pool sample was mixed with $100 \mu \mathrm{l}$ of an OT standard solution (10 000 $\mathrm{pg} / \mathrm{ml}$ ), extracted and serially diluted as described above. Dilutions of the spiked and unspiked pool sample were then brought to assay. Serially diluted pool samples of spiked and unspiked Barbary macaque urine were parallel to the standard as confirmed by visual inspection (Fig. $\mathrm{S} 1)$.

The assay standard curve ranged from 15.62 to $1000 \mathrm{pg} / \mathrm{ml}$ and assay sensitivity at $90 \%$ binding was $30 \mathrm{pg} / \mathrm{ml}$. Intra-assay coefficients of variation (CV) of high and low value quality controls (QC) were $5.2 \%$ (high) and $31.3 \%$ (low). In our assays, the QC low concentration was close to the lower limit of the linear range of the standard curve and this resulted in a high CV. A high CV increases noise in the dataset, but is unlikely to bias results towards (or against) our tested predictions. Only six samples (4\%) had a lower concentration than the average QC low, whereas most samples (90\%) had concentrations well within the linear range. Excluding the few samples that had low concentration of OT did not significantly impact our results. Inter-assay CVs were $11.0 \%$ (high) and $19.7 \%$ (low).

Urinary OT concentrations were corrected for levels of creatinine to account for differences in volume and concentration of excreted urine (Bahr et al., 2000), and are expressed as pg/mg creatinine. Because very low concentrations of creatinine may lead to an overestimation of hormone concentration we excluded all samples $(\mathrm{N}=3)$ with $<0.5 \mathrm{mg} / \mathrm{ml}$ creatinine.

\subsubsection{Statistical analysis}

To test whether affiliative interactions influenced urinary OT levels, we fitted two Bayesian multilevel linear regression models (model 1a, b) with a Gaussian response distribution and identity link function. To test whether the probability to give support in an agonistic encounter after being recruited was influenced by a previous grooming interaction, we fitted a Bayesian multilevel linear regression model with a Bernoulli response distribution and logit link function. We included male identity as a random effect in all models. In all models, predictor variables varied within male identity and therefore we included random slopes as well as correlation parameters between random intercepts and random slopes into the models (Barr et al., 2013; Schielzeth \& Forstmeier, 2009). We fitted models using the computational framework Stan (https://mc-stan.org), called via $\mathrm{R}$ (version 3.5.2; R Core Team, 2018) by using the function brm from the package brms (version 2.9.0; Bürkner, 2017). We ran all models with 5000 iterations over four MCMC chains including an initial 1000 "warm up" iterations for each chain, resulting in a total of 16000 posterior samples (Bürkner, 2017). In all models, we deemed the 
MCMC results as reliable because there were no divergent transitions during warm up, all Rhat values were equal to 1.00 and visual inspection of a plot of the chains showed that they were able to converge. We used a set of weakly informative priors to improve convergence, guard against overfitting and regularize parameter estimates (Lemoine, 2019; McElreath, 2016): for the intercept and beta coefficients we used a normal distribution with mean 0 and standard deviation 10; for the standard deviation of group level (random) effects and sigma we used a Half-Cauchy distribution with location 0 and scale parameter 1 ; for the correlation between random slopes we used LKJ Cholesky prior with eta 2.

For all models, we report the estimate as the mean of the posterior distribution and $95 \%$ credible intervals (CI). We calculated the proportion of the posterior samples that fall on the same side of 0 as the mean. This may be interpreted as the probability $(\operatorname{Pr})$ that a given predictor was associated with an outcome, where $\operatorname{Pr}=1$ indicates that the estimate was entirely positive or negative and $\operatorname{Pr}=0.5$ indicates that the estimate is centered around 0 and thus the predictor likely had no effect.

\subsubsection{Effect of affiliation on urinary OT levels (predictions 1 and 2)}

To test whether urinary OT levels were generally influenced by affiliative behaviors we fitted two models. As the response we log-transformed urinary OT levels to achieve a more symmetrical distribution. In model 1a, we tested for a general effect of affiliation (prediction 1) and included one categorical predictor where OT levels following grooming and triadic male-infant-male interactions were compared to non-social controls. In model $1 \mathrm{~b}$, we tested whether OT levels would be influenced differently after affiliation with bond versus non-bond partners (prediction 2). Therefore, we split samples after triadic male-infant-male interactions into bond and non-bond partner categories. As we were only able to collect two urine samples where focal males groomed with a non-bond partner, we decided not to split grooming samples according to partner bond strength.

\subsubsection{Effect of grooming on probability to give support (prediction 3)}

To test whether the probability to give support in an agonistic encounter (between adult and/or subadult individuals) was influenced by a previous grooming interaction, we fitted one model (model 2). To ensure that ongoing conflicts were salient to our focal males and account for the number of opportunities to provide agonistic support, we included whether our focal male supported another adult individual only following a recruit attempt (no/yes) as the response variable. As a test predictor, we included whether our focal animal was in a grooming interaction ( $\geq 60 \mathrm{sec}$ ) with an adult individual within 15-60 min before the recruit behavior (no/yes). This time window was chosen because intranasal administration of OT in rhesus macaques (Macaca mulatta) influenced social behaviors up to two hours after inhalation (Chang et al., 2012). Therefore, we chose a comparatively conservative window of 15-60 min for when naturally 
centrally released OT may still exert behavioral effects. As a control predictor, we included the affiliative relationship strength of the focal animal to the recruiter (non-bond/bond).

\subsection{Results}

\subsubsection{Effect of affiliation on urinary OT levels (predictions 1 and 2)}

We first tested for a general effect of affiliative interactions (grooming and male-infant-male triadic interactions) irrespective of partner bond strength on urinary OT levels. Urinary OT levels were substantially higher after grooming with any partner compared to non-social controls (mean \pm SD OT: non-social: $357 \pm 400 \mathrm{pg} / \mathrm{mg}$ creatinine; grooming: $589 \pm 612 \mathrm{pg} / \mathrm{mg}$ creatinine; average increase of $65 \%$; $\operatorname{Pr}=0.97$; Table $3.1 \mathrm{a}$, b; Fig. 3.1; Fig. 3.2), while this was not the case for male-infant-male interactions with any partner (mean \pm SD OT: male-infant-male: $455 \pm 438 \mathrm{pg} / \mathrm{mg}$ creatinine; $\operatorname{Pr}=0.77$; Table 3.1 ).

When we separated male-infant-male interaction samples by bond strength, urinary OT levels after male-infant-male interactions with bond partners were also not substantially different from non-social controls (mean \pm SD OT: non-social: $357 \pm 400 \mathrm{pg} / \mathrm{mg}$ creatinine; male-infant-male bond: $360 \pm 410 \mathrm{pg} / \mathrm{mg}$ creatinine). In contrast, urinary OT levels were substantially higher after triadic male-infant-male interactions with non-bond partners than non-social controls (mean \pm SD OT: male-infant-male non-bond: $600 \pm 456 \mathrm{pg} / \mathrm{mg}$ creatinine; average increase of $68 \%$; Pr $=0.96$; Table 3.1 b; Fig. 3.1; Fig. 3.2).

Table 3.1: Results of models $1 \mathrm{a}$ and $1 \mathrm{~b}$ testing effect of different affiliation conditions on urinary OT levels. In both models, male identity was included as a random effect, $\mathrm{N}=13$ males, $\mathrm{N}=$ 139 samples. $\mathrm{CI}=95 \%$ credible intervals, $\mathrm{Pr}=$ proportion of the posterior samples that fall on the same side of 0 as the mean.

\begin{tabular}{llllll}
\hline & Estimate & SD & CI lower & CI upper & Pr \\
\hline (a) & & & & & \\
$\quad$ Intercept & 5.48 & 0.12 & 5.25 & 5.71 & 1.00 \\
$\quad$ Groom & 0.47 & 0.25 & -0.01 & 0.99 & 0.97 \\
$\quad$ Male-infant-male & 0.17 & 0.23 & -0.30 & 0.60 & 0.77 \\
(b) & & & & & \\
$\quad$ Intercept & 5.49 & 0.11 & 5.26 & 5.70 & 1.00 \\
$\quad$ Groom & 0.47 & 0.25 & -0.01 & 1.00 & 0.97 \\
$\quad$ Male-infant-male bond & -0.05 & 0.28 & -0.59 & 0.51 & 0.58 \\
$\quad$ Male-infant-male non-bond & 0.57 & 0.33 & -0.09 & 1.22 & 0.96 \\
\hline
\end{tabular}



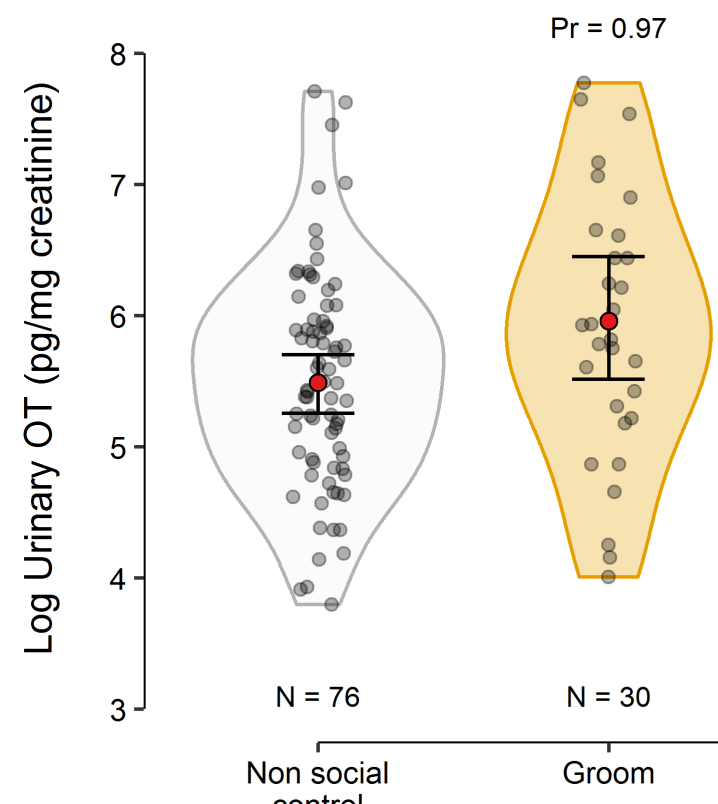

$\operatorname{Pr}=0.58$

$\mathrm{Pr}=0.96$

\section{吾}

Figure 3.1: Urinary OT levels per behavioral condition. Violin plots show the density of observed data points. Solid red dots show fitted values from model 1b: mean of posterior distribution and $95 \%$ credible intervals. $\operatorname{Pr}=$ proportion of the posterior samples that fall on the same side of 0 as the mean. $\mathrm{N}=$ number of samples per condition.

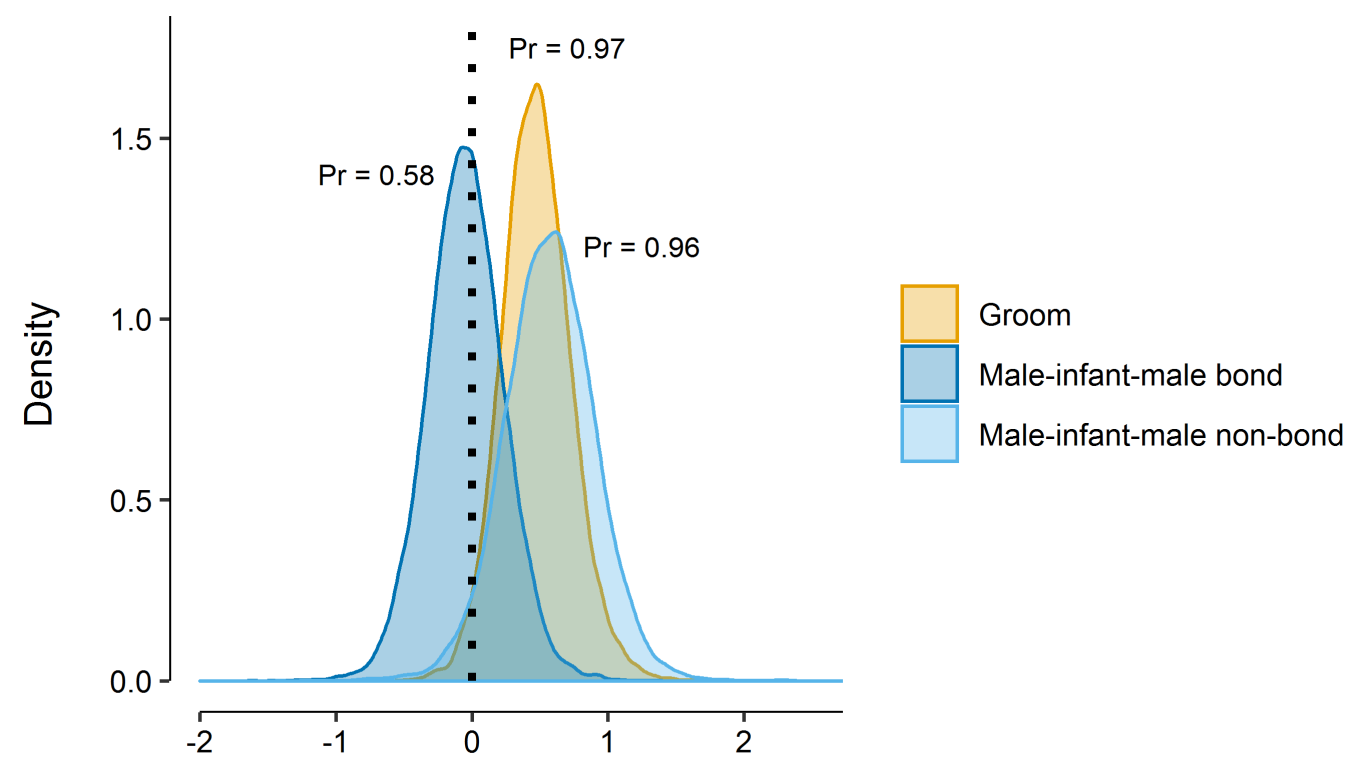

\section{Estimate}

Figure 3.2: Posterior probability distribution of the difference in urinary OT levels after different affiliative behaviors compared to non-social controls. $\mathrm{Pr}=$ proportion of the posterior samples that fall on the same side of 0 as the mean. 


\subsubsection{Effect of grooming on probability to give support (prediction 3)}

We recorded a total 205 attempts of adult individuals to recruit the focal animal for an agonistic conflict. In $64(31 \%)$ cases the focal animal supported the recruiter and in $67(33 \%)$ cases the focal animal was in a grooming interaction with any adult group member 15 to 60 minutes prior to the recruitment attempt (these samples are not mutually exclusive). In only 7 (3\%) cases were the previous grooming partner also the recruiter. Grooming interactions did not substantially influence the probability to support a recruiter in an agonistic encounter within 15 to 60 minutes after the grooming interaction ( $\mathrm{N}$ support given when groomed before $=16$, $\mathrm{N}$ support given when not groomed before $=48 ; \operatorname{Pr}=0.86$; Table 3.2).

Table 3.2: Model 2 results testing the effect of grooming on the probability to give support in an agonistic conflict after being recruited. Bond strength with the recruiter was included as a control variable. $\mathrm{N}=14$ males, $\mathrm{N}=205$ observations. $\mathrm{CI}=95 \%$ credible intervals, $\mathrm{Pr}=$ proportion of the posterior samples that fall on the same side of 0 as the mean.

\begin{tabular}{llllll}
\hline & Estimate & SD & CI lower & CI upper & Pr \\
\hline Intercept & -1.05 & 0.35 & -1.80 & -0.41 & 1.00 \\
Groom before? (yes) & -0.45 & 0.43 & -1.32 & 0.37 & 0.86 \\
Recruiter bond (bond) & 0.81 & 0.46 & -0.03 & 1.82 & 0.97 \\
\hline
\end{tabular}

\subsection{Discussion}

Overall we found a high probability that urinary OT levels were elevated following grooming interactions in adult male Barbary macaques. This is generally in line with previous studies showing a positive relationship between OT and grooming (primates: Benítez et al., 2018; Crockford et al., 2013; Snowdon et al., 2010; vampire bats, Desmodus rotundus: Carter \& Wilkinson, 2015), as well as other socio-positive interactions more generally (primates: Benítez et al., 2018; Moscovice et al., 2019; Preis et al., 2018; Snowdon et al., 2010; Wittig et al., 2014; vampire bats: Carter \& Wilkinson, 2015; dogs: Romero et al., 2014). Given that grooming was rare among non-bonded partners and therefore unlikely to occur in the excretion window before a urine sample was collected, we could not test partner specific effects of grooming. Results on partner-specificity of OT release are inconsistent. In one population of chimpanzees, OT increased only after grooming with a bond, yet not with a non-bond partner (Crockford et al., 2013), while in bonobos and in another chimpanzee population OT levels were generally increased after affiliation irrespective of partner relationship strength (Moscovice et al., 2019; Preis et al., 2018). Relationship quality was tested differently in the two chimpanzee studies, with relationship quality being either categorized dichotomously into bond and non-bond partners (Crockford et al., 2013), or being assayed on a continuous scale (Preis et al., 2018). Our cut-off relationship strength value for classification as a bond partner was much lower than the one used for chimpanzees. We do not know how nonhuman primates classify each 
other into biologically meaningful bond and non-bond categories, e.g. an inner clique of 2-3 bonded partners (Hill et al., 2008; Zhou et al., 2005), and if this mental classification mediates OT release. In principle there is good evidence that classification into bond partners affects physiological responses to social interactions. The social buffering phenomenon shows that the presence or interaction with closely bonded partners during stressful events mitigates the release of glucocorticoids (Hostinar et al., 2014; Wittig et al., 2016; Young et al., 2014a) with OT release mediating social buffering of the stress response (Crockford et al., 2017; Hostinar et al., 2014; Smith \& Wang, 2014).

Unexpectedly, urinary OT levels were elevated after triadic male-infant-male interactions with non-bond partners, but not after interactions with bond partners. This finding contradicts the idea that it is affiliation with bond rather than non-bond partners that triggers the release of OT (Crockford et al., 2013; Finkenwirth et al., 2015; but see Moscovice et al., 2019; Preis et al., 2018). This could indicate that male-infant-male interactions serve to promote the formation of social bonds with not yet bonded partners, while physiologically not impacting interactions between established partners. An untested, yet possible alternative given the correlational nature of our study is the reversed cause-effect directionality: instead of a male-infant-male interaction triggering the release of OT, OT may increase the probability of a male-infant-male interaction to occur. In this scenario, male-infant-male interactions do not function in bond formation, but for other reasons, for example as a form of "agonistic buffering" (Deag, 1980; Deag \& Crook, 1971; Paul et al., 1996). In support of this idea, rates of male-infant-male interactions increase during tense feeding situations while other types of affiliation (such as grooming) decrease (Paul et al., 1996). Social relationships between adult males are generally tense and affiliation between them often takes place in the presence of infants (Deag, 1980; Preuschoft \& Paul, 2000). Due to the anxiolytic effect of OT (Neumann \& Landgraf, 2012), elevated levels of OT may facilitate male-infant-male interactions via increasing the motivation to approach and at the same time reducing avoidance behaviors toward other males (Kemp \& Guastella, 2011). Reduced anxiety may be particularly useful for interacting with non-bond males with whom the relationship is presumably more tense and unpredictable than with bond partners (Young et al., 2014b). Such an explanation is consistent with our finding that OT levels were elevated after male-infant-male interactions with non-bond partners. Similarly, same-sex genital rubbing among female bonobos is associated with increased OT levels and may also function to reduce social conflict (Moscovice et al., 2019). While the role for OT and male-infant-male interactions in agonistic buffering is intriguing, we need to stress our small sample size of interactions with non-bond partners as well as previous work on male macaques pointing to the bond strengthening and cooperation-enhancing function of male-infant-male interactions (Berghänel et al., 2011a; Kalbitz et al., 2017). Thus, additional studies able to disentangle OT levels directly before and after affiliative interactions are clearly needed for a more conclusive picture.

Prior grooming did not increase the probability of supporting a group member in a conflict. This test builds on the assumption that after engaging in grooming OT levels will be elevated, potentially influencing behavior and more specifically cooperative tendency. If true, this finding 
suggests that OT did not increase a male's general tendency to cooperate in a conflict, which is maybe not surprising given the wealth of studies indicating that OT's prosocial effects depend on situational context and interaction partner (Bartz et al., 2011). In an economic game, human cooperation was enhanced by intranasal OT administration only if participants had prior contact yet not with strangers (Declerck et al., 2010), and similarly, female house mice (Mus musculus domesticus) receiving OT actually decreased the propensity to cooperate in communal breeding with strangers (Harrison et al., 2017). In another study in humans intranasal OT increased trust but not if the partner was portrayed as untrustworthy (Mikolajczak et al., 2010). Thus, the social information on a partner is an important component for OT-induced cooperation and depending on this information OT may reduce the propensity to cooperate.

Our results do not exclude the possibility that OT promotes direct or partner-specific cooperation. From a behavioral perspective, individuals are more likely to support others with whom they have groomed in the recent past (long-tailed macaques, Macaca fascicularis: Hemelrijk, 1994; chacma baboons, Papio ursinus: Cheney et al., 2010). In our study there were only a few cases of the former grooming partner asking for help within the next hour, therefore we could not explicitly test this scenario. Contingent cooperation appears to be rare in animals, and more commonly support is given less strictly on a contingent basis but instead to bonded partners who form long term alliances (Cheney, 2011). OT would then mediate cooperation with specific partners through its role in promoting the formation of social bonds. While we did not have an explicit aim to test the effect of affiliative relationship strength on the probability to cooperate, this variable was included in our model as a control predictor, and it did substantially increase the probability of giving support. Preferentially giving support to bonded partners has also previously been shown in this (Young et al., 2014b) and other species (Schülke et al., 2010; Smith et al., 2011; Watts, 2002).

In this study, we measured peripheral oxytocin in urine samples. Oxytocin is produced in the hypothalamus and is released both centrally and in the periphery (Landgraf \& Neumann, 2004). As OT does not readily cross the blood-brain barrier (Landgraf \& Neumann, 2004), there is an ongoing debate about whether peripheral measures of OT reflect central levels, since central and peripheral release can be coordinated or independent (Jurek \& Neumann, 2018). However, there is accumulating support that peripheral measures of OT indeed reflect central levels. Induced central OT release shows detectable changes in peripheral matrices such as blood and urine (Francis et al., 2016). Across species, a growing number of studies, including this one, found that peripheral OT correlates with predicted changes in behavior including in affiliative and cooperative contexts (e.g. Benítez et al., 2018; Moscovice et al., 2019; Samuni et al., 2017; reviewed in Crockford et al., 2014). Combined these studies suggest that changes in central OT levels correspond to changes in peripheral OT levels. However, to conclusively answer this we need experimental studies that simultaneously measure central and peripheral OT release (e.g. Wotjak et al., 1998).

Overall, our study adds to the body of research indicating that OT is involved in the regulation of adult social bonds, including in non-reproductive contexts. Questions still remain under which 
contexts OT release is partner specific. It has been suggested that in smaller social groups, all group members are bonded to a sufficient degree to elicit OT release after affiliative interactions (Benítez et al., 2018), whereas in larger groups variance in affiliation rates may be large enough that OT release may only occur after affiliations with more closely bonded partners. Such an explanation would be consistent with observed differences in partner specificity of OT release between chimpanzee populations (Crockford et al., 2013; Preis et al., 2018). Finally, the lack of an effect of male-infant-male interactions on OT levels in male Barbary macaques, at least with bond partners and perhaps overall, remains puzzling. Particularly so given that behaviors other than grooming that potentially promote social bonding also increased urinary OT levels in other species (Benítez et al., 2018; Romero et al., 2014; Wittig et al., 2014). One difference between grooming and male-infant-male interactions is that male-infant-male interactions are more ritualistic in nature. Perhaps the bonding effect of ritualized behavior may be under the control of other neuropeptides, such as endorphins, as has been shown for synchronous dancing in humans (Tarr et al., 2015, 2016).

\section{Acknowledgements}

We thank Ellen Merz and Roland Hilgartner for permission to conduct the study at Affenberg Salem. We thank Lauren Cassidy, Tatjana Kaufmann and Lilah Sciaky for help with urine sample and behavioral data collection. We are grateful to Vera Schmeling for general assistance in the hormone laboratory. This study benefitted from stimulating discussions with members of the DFG-research unit "Sociality and Health in Primates" (DFG FOR 2136). This research was funded by the Deutsche Forschungsgemeinschaft (DFG, German Research Foundation) Project number 254142454 / GRK 2070. 
CHAPTER FOUR 


\section{Chapter 4}

\section{Testosterone and cortisol are negatively associated with ritualized bonding behavior in male Barbary macaques}

Alan V. Rincon ${ }^{\mathrm{a}, \mathrm{b}}$, Michael Heistermann ${ }^{\mathrm{c}}$, Oliver Schülke $\mathrm{e}^{\mathrm{a}, \mathrm{b}, \mathrm{d}}$, Julia Ostner ${ }^{\mathrm{a}, \mathrm{b}, \mathrm{d}}$

${ }^{a}$ Department of Behavioral Ecology, Johann-Friedrich-Blumenbach Institute for Zoology and Anthropology, University of Goettingen, Goettingen, Germany

${ }^{b}$ Leibniz ScienceCampus Primate Cognition, Goettingen, Germany

${ }^{c}$ Endocrinology Laboratory, German Primate Center, Leibniz Institute for Primate Research, Goettingen, Germany

${ }^{d}$ Research Group Social Evolution in Primates, German Primate Center, Leibniz Institute for Primate Research, Goettingen, Germany

Psychoneuroendocrinology Under review. 


\section{Abstract}

Neuroendocrine research on the formation of social bonds has primarily focused on the role of nonapeptides, however, steroid hormones often act additionally to either inhibit or facilitate bonding. Testosterone is proposed to mediate a trade-off between male reproductive competition and nurturing; therefore, low levels are predicted during periods of nurturing infant care and social bonding. In species where social bonding and support regulates hypothalamic-pituitary-adrenal axis activity, we also expect glucocorticoid levels to be low during bonding periods. Here we investigated how urinary testosterone and cortisol were related to male-infant-male interactions - a ritualized male bonding behavior - as well as infant care in male Barbary macaques (Macaca sylvanus). We collected $>3000$ hours of behavioral observation data during full-day focal animal follows from 14 adult males and analyzed testosterone and cortisol levels from 650 urine samples. As predicted both testosterone and cortisol levels were negatively correlated with rates of male-infant-male interactions. Infant care was positively correlated to testosterone and cortisol levels. The observed negative relationship between testosterone male-infant-male interactions may be beneficial to lower competitive tendencies between adult males and to not inhibit bond formation. Concurrently, lowered cortisol could reflect increased bonding and perceived social support as male-infant-male interactions predict future coalition formation in this species. Alternatively, lowered cortisol levels may be reflective of buffered tensions between males. The positive relationship of testosterone and cortisol with infant care suggests it may be done under competitive rather than nurturing contexts in this species. Collectively our results suggest that low steroid levels may be beneficial to same-sex bonding. Measuring steroid hormones in relation to bonding and nurturing can help us interpret behaviors within the ecological contexts that they occur.

Keywords: Steroid/peptide theory, testosterone, cortisol, social bond, male-infant-male interaction, Barbary macaque 


\section{$4.1 \quad$ Introduction}

Steroid and nonapeptide hormones are involved in regulating animal behavior with the potential for cross-communication between different regulatory axes, allowing for fine-tuning of behavioral responses to different social contexts (Neumann, 2002; van Anders et al., 2011; Viau, 2002). The steroid/peptide theory of social bonds ( $\mathrm{S} / \mathrm{P}$ theory) proposes that testosterone (regulated by the hypothalamic-pituitary-gonadal (HPG) axis) and oxytocin (regulated by the oxytocinergic system) interact to promote different behavioral goals (van Anders et al., 2011). The oxytocinergic system likely evolved in mammals to promote mother-offspring bonds, yet the system was co-opted to also promote bonds between adults in both sexual and non-sexual contexts (Numan \& Young, 2016). The HPG axis regulates reproduction, with testosterone the major androgen produced in males - promoting sexual contact and competition (Wingfield et al., 1990). Thus, while high oxytocin levels promote bonding and intimacy, variation in testosterone levels in conjunction with high oxytocin should be sensitive to different types of intimacy (e.g. sexual versus nurturing intimacy; van Anders et al. (2011)).

The S/P theory (van Anders et al., 2011) builds on the challenge hypothesis (Wingfield et al., 1990), proposing that high testosterone levels promote male reproductive competition and is traded-off against paternal care. Across vertebrates males exhibit elevated testosterone levels when competition for mates is high, such as during the mating season or territorial defense, and low testosterone levels during periods of paternal care (Hirschenhauser \& Oliveira, 2006; Lynn, 2008; Muller, 2017; Wingfield et al., 1990). Testosterone potentially inhibits paternal care as experimentally increasing testosterone levels reduces paternal behavior in various bird species (Lynn, 2008), including increases are within an individual's natural range (Goymann \& Flores, 2017). However, testosterone levels are positively related to paternal care when care is competitive in nature, e.g. offspring defense (Moore et al., 2019; Muller, 2017). The S/P theory resolves this inconsistency in how testosterone relates to paternal care by proposing that high testosterone levels inhibit nurturing behaviors and intimacy in general rather than paternal care per se (van Anders, 2013; van Anders et al., 2011).

Beyond competition and nurturing care, testosterone is relevant to bonding between same-sex adults. Same-sex adult bonds are usually characterized by affiliative contact and mutual social support (Massen et al., 2010; Ostner \& Schülke, 2018) and share many qualities with nurturing infant care. Consequently, the $\mathrm{S} / \mathrm{P}$ theory proposes that the ideal physiological conditions to induce bond formation are high levels of oxytocin coupled with low levels of testosterone (van Anders et al., 2011). Consistent with this, high oxytocin and lowered testosterone have been linked to meat-sharing in male chimpanzees (Pan troglodytes: Sobolewski et al., 2012; Wittig et al., 2014), a behavior implicated in bond formation (Wittig et al., 2014). Similarly, both low basal levels of testosterone, as well as decreased levels relative to baseline, were associated with feelings of closeness following a friendship formation task in same-sex adult human dyads (Ketay et al., 2017).

The HPG axis and the oxytocinergic system are also involved in cross-communication with the 
hypothalamic-pituitary-adrenal (HPA) axis (Neumann, 2002; Viau, 2002), including in contexts relevant to social bonds. The presence of or interaction with bonded partners during a potentially stressful event often mitigates HPA axis activity, a phenomenon known as social buffering that may be moderated by oxytocin (Hostinar et al., 2014). Being well socially integrated or being able to count on others for social support may help to regulate HPA axis activity even in the immediate absence of stressors (Rosal et al., 2004; Wittig et al., 2016). As with testosterone, cortisol levels also decline in humans after increasing feelings of personal closeness, and individuals with low cortisol had partners that desired to be closer to them (Ketay et al., 2017). Thus, while not explicitly part of the S/P theory, mutually lowered cortisol and testosterone may have synergistic facilitative effects in the context of same-sex bonding.

The aim of this study was to test predictions of the S/P theory (van Anders et al., 2011) regarding nurturing and bonding in male Barbary macaques (Macaca sylvanus) in relation to testosterone levels. Given the potential cross-talk between the HPG axis and the oxytocinergic system with the HPA axis, we also explored the relationship between GCs with nurturing and bonding. Male Barbary macaques form stable social bonds with other males (Young et al., 2014b) that physiologically buffer bonded males during stressful situations (Young et al., 2014a). Two types of interactions are relevant to the $\mathrm{S} / \mathrm{P}$ theory; triadic male-infant-male interactions and infant care. Male-infant-male interactions are ritualistic and involve two males sitting in body contact with an infant in-between them, exchanging affiliative facial signals and often inspecting the infant's genitals (Deag \& Crook, 1971). They occur primarily during the non-mating season, predict coalition formation in the following mating season, and have been implicated in bond formation (Berghänel et al., 2011a; Kalbitz et al., 2017). Male-infant-male interactions may also function in 'agonistic buffering', where the presence of the infant allows two males to approach each other in a non-threatening context (Deag \& Crook, 1971; Paul et al., 1996). As per the S/P theory, we predicted high oxytocin and low testosterone levels to be associated with male-infant-male interactions. In a previous study, we found mixed results for a positive relationship between male-infant-male interactions and oxytocin levels (Rincon et al., 2019a). Here, we tested the other half of the S/P theory and predicted that urinary testosterone levels would be negatively correlated with rates of male-infant-male interactions. Given the potential bonding and/or agonistic buffering function of male-infant-male interactions, we additionally predicted a negatively correlation of this behavior with urinary cortisol levels.

Infant care is the second interaction type relevant to the S/P theory; it involves huddling, carrying, and grooming infants (Whitten, 1987), which are all nurturing in nature. We therefore predicted urinary testosterone levels to be negatively correlated with infant care. As male infant care was positively correlated with fecal GC levels on a previous study on the same species (Henkel et al., 2010), we predicted a positive link between infant care and urinary cortisol. 


\subsection{Materials and methods}

\subsubsection{Study site and animals}

Study subjects belonged to one group of Barbary macaques (group C) living in semi-free ranging conditions within 14.5 ha of enclosed forest at Affenberg Salem, Germany (de Turckheim \& Merz, 1984). Monkeys were provisioned once a day with fruits, vegetables, grains and had ad libitum access to water and monkey chow. Data were collected from 31 March to 26 October 2016 during one non-mating season. The study group consisted of 14 adult males ( 7 to 25 years old), 20 adult females ( 5 to 27 years old), 2 large subadult males (6 years old), 8 immature males ( 1 to 4 years old), 10 immature females ( 1 to 4 years old) and 1 newborn infant male ( $<1$ years old). The oldest adult male died during the study period on 20 August 2016. All group members could be individually recognized by observers though a combination of unique characteristics including body size, coat color and condition, facial spots and scars, gait and identification tattoos on the inner thigh.

\subsubsection{Behavioral data collection and dominance hierarchy}

Behavioral data were collected from 14 adult males using continuous focal animal sampling (Martin \& Bateson, 2007) during individual full-day focal animal follows (total $=3289$ hours, mean \pm standard deviation $=235 \pm 19$ hours per individual). The occurrence of all social interactions, including the identity of social partner(s) were recorded.

To construct a dominance hierarchy, we used agonistic interactions recorded during continuous focal animal sampling and ad libitum sampling. An agonistic interaction was defined by the occurrence of at least one aggressive (open-mouth threat, stare, lunge, charge, chase, physical aggression) and/or submissive (make room, give ground, flee) behavior. Aggressive and submissive behaviors occurring in quick succession of each other were considered as part of the same conflict. We calculated one dominance hierarchy for the entire study period using normalized David's score (de Vries et al., 2006) in R (version 3.5.3; R Core Team, 2018) using the function DS from the package EloRating [version 0.46.8]. A higher David's score indicates higher dominance rank. The dominance hierarchy was stable throughout the whole study period.

\subsubsection{Urine sample collection}

Urine samples were collected from focal males whenever possible by catching it with a plastic bag or otherwise from leaves, plant litter or ground, using a disposable plastic pipette to transfer the sample to $2 \mathrm{ml}$ cryotubes. When pipetting was not possible we absorbed urine using a salivette 
Table 4.1: Descriptive information per study subject for the study period

\begin{tabular}{llllllll}
\hline $\begin{array}{l}\text { Male } \\
\text { ID }\end{array}$ & $\begin{array}{l}\text { Obs. } \\
\text { days }\end{array}$ & $\begin{array}{l}\text { Obs. } \\
\text { hours }\end{array}$ & $\begin{array}{l}\text { Dominance } \\
\text { rank (norm } \\
\text { David's score) }\end{array}$ & $\begin{array}{l}\text { Male-infant-male } \\
\text { interactions } \\
\text { (per obs. hr.) }\end{array}$ & $\begin{array}{l}\text { Infant care } \\
\text { (\% obs. time) }\end{array}$ & $\begin{array}{l}\text { Age } \\
\text { (years) }\end{array}$ & $\begin{array}{l}\text { Urine } \\
\text { samples } \\
\text { (N) }\end{array}$ \\
\hline W3 & 29 & 237 & 14.7 & 0.85 & 3.46 & 12 & 24 \\
U2 & 29 & 242 & 13.1 & 0.47 & 1.17 & 13 & 42 \\
M3 & 30 & 234 & 12.6 & 0.06 & 2.17 & 21 & 20 \\
T3 & 31 & 242 & 11.1 & 0.59 & 2.42 & 14 & 56 \\
M4 & 30 & 247 & 10.9 & 0.10 & 0.09 & 21 & 49 \\
W7 & 28 & 223 & 10.3 & 0.33 & 0.66 & 12 & 44 \\
B5 & 30 & 237 & 7.9 & 1.93 & 6.70 & 7 & 66 \\
B6 & 29 & 247 & 7.3 & 0.66 & 2.14 & 7 & 61 \\
B7 & 30 & 240 & 7.2 & 1.75 & 3.25 & 7 & 49 \\
Z5 & 28 & 238 & 5.2 & 0.09 & 0.15 & 9 & 49 \\
Z6 & 28 & 238 & 4.4 & 0.37 & 1.19 & 9 & 73 \\
A6 & 28 & 237 & 3.9 & 0.19 & 0.06 & 8 & 59 \\
I1 & 14 & 171 & 2.2 & 0.01 & 0.03 & 25 & 13 \\
B1 & 28 & 244 & 2.0 & 0.28 & 0.19 & 7 & 45 \\
\hline
\end{tabular}

(Salivette Cortisol, Sarstedt, Nümbrecht, Germany) (Danish et al., 2015). Samples were only collected if they were not contaminated with fecal matter or urine from other individuals. Both samples in cryotubes and salivettes were stored in a thermos container filled with ice while in the field. At the end of the day, samples collected by salivette were centrifuged at $1500 \mathrm{rpm}$ for 5 minutes to extract the urine, which was afterwards transferred to $2 \mathrm{ml}$ cryotubes. All samples were then stored in a freezer at $-20^{\circ} \mathrm{C}$. At the end of the field season samples were transported on dry ice to the University of Göttingen where they were stored again at $-20^{\circ} \mathrm{C}$ until analysis.

Based on radiometabolism studies assessing the time for hormone excretion in macaque urine, we considered behaviors that occurred up to six hours prior to sample collection to affect urinary testosterone levels (Möhle et al., 2002) and behaviors that occurred within the same day (up to 13.5 hours) prior to sample collection to affect urinary cortisol levels (Bahr et al., 2000). We excluded samples from which we had $<2$ hours of observation time prior to sample collection ( $\mathrm{N}$ $=60$ ), leaving a total of 650 urine samples for analysis. Table 4.1 shows descriptive information for each study subject.

\subsubsection{Hormone analysis}

The measurement of cortisol and testosterone from the urine of male Barbary macaques has been previously validated (Rincon et al., 2019b). For the accurate quantification of testosterone, but not cortisol, urine samples needed to be enzymatically hydrolyzed prior to hormone measurement (Rincon et al., 2019b). Hydrolysis was carried out by mixing 20 - $100 \mu \mathrm{l}$ urine of each sample with $40 \mu \mathrm{l} \beta$-Glucuronidase (K12 strain Escherichia coli, Prod. No. BGALS-RO, Sigma-Aldrich) and $900 \mathrm{\mu l}$ phosphate buffer (composed of $6.0 \mathrm{~g} \mathrm{NaH}_{2} \mathrm{PO}_{4} \times \mathrm{H}_{2} \mathrm{O}+14.5 \mathrm{~g} \mathrm{Na}_{2} \mathrm{HPO}_{4} \times 2 \mathrm{H}_{2} \mathrm{O}$ dissolved in $500 \mathrm{ml}$ water; $\mathrm{pH}$ 6.9) and incubating in a shaking water bath at $37^{\circ} \mathrm{C}$ overnight. 
Hydrolyzed samples were then purified and extracted using solid phase extraction cartridges (Chromabond HR-X, 30mg, 1ml, Macherey-Nagel, Dueren, Germany) placed onto a 12-port vacuum manifold. Prior to sample loading, cartridges were conditioned first with $1 \mathrm{ml}$ of $\mathrm{MeOH}$, followed by $1 \mathrm{ml} \mathrm{H}_{2} \mathrm{O}$ and $1 \mathrm{ml}$ phosphate buffer. Columns were not allowed to run dry at this step. Hydrolyzed urine samples were then pipetted into pre-labelled cartridges and the solution was let to sink in. Cartridges were subsequently rinsed three times with $1 \mathrm{ml} \mathrm{H}_{2} \mathrm{O}$ followed by two times with $1 \mathrm{ml} \mathrm{MeOH} / \mathrm{H}_{2} \mathrm{O}$ (40/60) solution, after which cartridges were dried by applying a vacuum. Steroids were finally eluted with $1 \mathrm{ml}$ absolute $\mathrm{MeOH}$ followed by $1 \mathrm{ml}$ ethyl acetate, and eluates were then evaporated to dryness at $40^{\circ} \mathrm{C}$ under pressurized air. Then, $1 \mathrm{ml}$ hydrolysis phosphate buffer was added to the dried sample eluates and vortexed for $5 \mathrm{~min}$. Afterwards $150 \mathrm{\mu l} 10 \% \mathrm{~K}_{2} \mathrm{CO}_{3}$ buffer and $5 \mathrm{ml}$ tert. butyl methyl ether (TBME) was added to each tube for extraction of the liberated steroids. Tubes were sealed with caps and parafilm, vortexed for $10 \mathrm{~min}$ on a multi-tube vortexer, then centrifuged for $5 \mathrm{~min}$ at $2000 \mathrm{rpm}$ and subsequently store at $-20^{\circ} \mathrm{C}$ until the aqueous layer had frozen out. The ether phase was then poured into new collection tubes, the ether was evaporated to around $1 \mathrm{ml}$, and briefly vortexed to concentrate steroids in the bottom of the tube, and finally evaporated to dryness. Dried samples were reconstituted in $1 \mathrm{ml}$ absolute $\mathrm{MeOH}$ by vortexing for $10 \mathrm{~min}$. Extracts were then transferred into $2 \mathrm{ml}$ eppendorf safe-lock tubes and stored at $-20^{\circ} \mathrm{C}$ until analysis for testosterone concentrations. The efficiency of the combined hydrolysis and extraction procedure was assessed using internal controls of testosterone glucuronide run together with each set of samples as described earlier (Rincon et al., 2019b). The combined hydrolysis/extraction efficiency was 62.1 $\pm 4.2 \%$ mean $\pm \mathrm{SD}$ (range: $50-70 \% ; \mathrm{N}=36$ ).

We measured immunoreactive urinary testosterone from samples following extraction and hydrolysis (described above), and immunoreactive urinary cortisol from unprocessed diluted urine by using enzyme immunoassays as described in detail in Rincon et al. (2019b). For the testosterone assay, sensitivity at $90 \%$ binding was $3.0 \mathrm{pg}$; intra-assay CVs of high and low value quality controls were $<10 \%$, while figures for inter-assay CVs were $13.2 \%$ (high) and $19.5 \%$ (low). For the cortisol assay, sensitivity at $90 \%$ binding was $0.6 \mathrm{pg}$; intra-assay CVs of high and low value quality controls were $<10 \%$, while figures for inter-assay CVs were $9.2 \%$ (high) and $10.1 \%$ (low). To account for differences in the volume and concentration across urine samples, urinary testosterone and cortisol levels were corrected for creatinine and are expressed as ng/mg Cr (Bahr et al., 2000).

\subsubsection{Statistical analyses}

To test whether male-infant-male interactions influenced urinary testosterone and cortisol levels, we fitted two Bayesian multilevel linear regression models with a Gaussian response distribution and identity link function. Urinary testosterone (model 1) and urinary cortisol (model 2) were our response variables. Both hormone values had a strong positive skew so we did a natural log transformation to achieve a more symmetrical distribution and used these values in analysis. Our test predictors included a count of male-infant-male interactions and male infant care, defined 
as the time males spent in body contact with infants including huddling, carrying and grooming in both models. We included duration of grooming, count of male-male aggression, dominance rank (normalized David's score), and time of sample collection (daytime) as control predictors in both models. Counts of male-infant-male interactions and aggression, and duration of infant care were corrected for the observation time of the urine sample window. Each male had one dominance rank score for the entire study period (Table 4.1). All predictors were z-transformed to a mean of 0 and standard deviation of 1 to improve model convergence and interpretation of results.

We fitted models in $\mathrm{R}$ (version 3.5.2; $\mathrm{R}$ Core Team, 2018) using the function brm from the package brms (version 2.9.0; Bürkner, 2017). The package brms calls on the computational framework Stan (https://mc-stan.org) to fit Bayesian models (Bürkner, 2017). We included male identity as a random effect in all models to control for multiple observations (urine samples) per subject. We also included random slopes and correlation parameters between random intercepts and random slopes for all predictors because they had at least three unique values in over half of subjects (Barr et al., 2013). However, we had to exclude correlations between random intercepts and random slopes in the testosterone model (model 1) to ensure there were no divergent transitions after warm-up. We ran all models with 5000 iterations over four MCMC chains, which included 1000 "warm up" iterations for each chain, resulting in a total of 16000 posterior samples (Bürkner, 2017). In all models, there were no divergent transitions during warm up, all Rhat values were equal to 1.00 and visual inspection of a plot of the chains showed that they were able to converge. We used weakly informative priors to improve convergence, guard against overfitting and regularize parameter estimates (McElreath, 2016). As a prior for the intercept and beta coefficients we used a normal distribution with mean 0 and standard deviation 10; for the standard deviation of group level (random) effects and sigma we used a Half-Cauchy distribution with location 0 and scale parameter 1; for the correlation between random slopes we used LKJ Cholesky prior with eta 2 .

We report model estimates as the mean of the posterior distribution with $95 \%$ credible intervals (CI). To decide whether predictor variables were substantially affecting the response (hormone levels), we calculated the proportion (Pr) of posterior samples that fell on the same side of 0 as the mean. The Pr ranges from 0.5 to 1.0 , with $\mathrm{Pr}=1.00$ indicating that, given the model, the effect of a predictor was entirely positive or negative, whereas $\operatorname{Pr}=0.5$ indicates that the effect was centered around 0 . We considered $\operatorname{Pr}>0.95$ as substantial evidence that a predictor was associated with an outcome.

\subsection{Results}

We recorded a total of 1701 triadic male-infant-male interactions during 3289 hours of observation in one non-mating season. All males of the study group were observed to partake in male-infant-male interactions at least once (Table 4.1). The majority (64\%) of male-infant-male 
Table 4.2: Urinary testosterone levels in relation to male-infant-male interactions and male infant care (model 1). Male identity was included as a random effect, $\mathrm{N}$ subjects $=14, \mathrm{~N}$ urine samples $=650 . \mathrm{CI}=95 \%$ credible intervals, $\mathrm{Pr}=$ proportion of the posterior samples that fall on the same side of 0 as the mean.

\begin{tabular}{lccccc}
\hline & Estimate & SD & CI lower & CI upper & $\operatorname{Pr}$ \\
\hline $\begin{array}{l}\text { Intercept } \\
\text { Test predictors }\end{array}$ & 3.04 & 0.09 & 2.85 & 3.22 & 1.00 \\
$\quad$ & & & & & \\
$\quad$ Male-infant-male & -0.18 & 0.06 & -0.32 & -0.07 & $>0.99$ \\
$\quad$ Infant care & 0.10 & 0.05 & -0.00 & 0.20 & 0.97 \\
Control predictors & & & & & \\
$\quad$ Grooming & 0.03 & 0.04 & -0.04 & 0.11 & 0.82 \\
$\quad$ Aggression & -0.02 & 0.04 & -0.10 & 0.06 & 0.68 \\
$\quad$ Dominance rank & -0.17 & 0.08 & -0.33 & -0.01 & 0.98 \\
$\quad$ Daytime & -0.03 & 0.03 & -0.09 & 0.04 & 0.79 \\
\hline
\end{tabular}

Table 4.3: Urinary cortisol levels in relation to male-infant-male interactions and male infant care (model 2). Male identity was included as a random effect, $\mathrm{N}$ subjects $=14, \mathrm{~N}$ urine samples $=650 . \mathrm{CI}=95 \%$ credible intervals, $\mathrm{Pr}=$ proportion of the posterior samples that fall on the same side of 0 as the mean.

\begin{tabular}{lccccc}
\hline & Estimate & SD & CI lower & CI upper & Pr \\
\hline Intercept & 5.17 & 0.12 & 4.94 & 5.42 & 1.00 \\
Test predictors & & & & & \\
$\quad$ Male-infant-male & -0.32 & 0.15 & -0.67 & -0.08 & 0.99 \\
$\quad$ Infant care & 0.08 & 0.05 & -0.02 & 0.20 & 0.95 \\
Control predictors & & & & & \\
$\quad$ Grooming & 0.00 & 0.06 & -0.12 & 0.12 & 0.53 \\
$\quad$ Aggression & -0.03 & 0.03 & -0.10 & 0.03 & 0.83 \\
$\quad$ Dominance rank & -0.18 & 0.10 & -0.38 & 0.01 & 0.97 \\
$\quad$ Daytime & -0.23 & 0.04 & -0.31 & -0.15 & 1.00 \\
\hline
\end{tabular}

interactions involved the one newborn male, $27 \%$ involved yearlings and $9 \%$ involved juveniles. Rates of male-infant-male interactions were negatively correlated with both testosterone (Table 4.2; Fig. $4.1 a, b$ ) and cortisol (Table 4.3; Fig. 4.2, $a, b$ ) levels.

As with triadic interactions, all males of the group were observed caring for infants ( $\leq 1$ years old), including time spent huddling, carrying, or grooming (Table 4.1). Male infant care was positively correlated with both testosterone (Table 4.2; Fig. 4.1, a, c) and cortisol (Table 4.3; Fig. 4.2, $a, c)$ levels.

\subsection{Discussion}

In this study, we investigated how testosterone and cortisol levels related to social bonding and nurturing in male Barbary macaques, testing predictions of the steroid/peptide theory of 

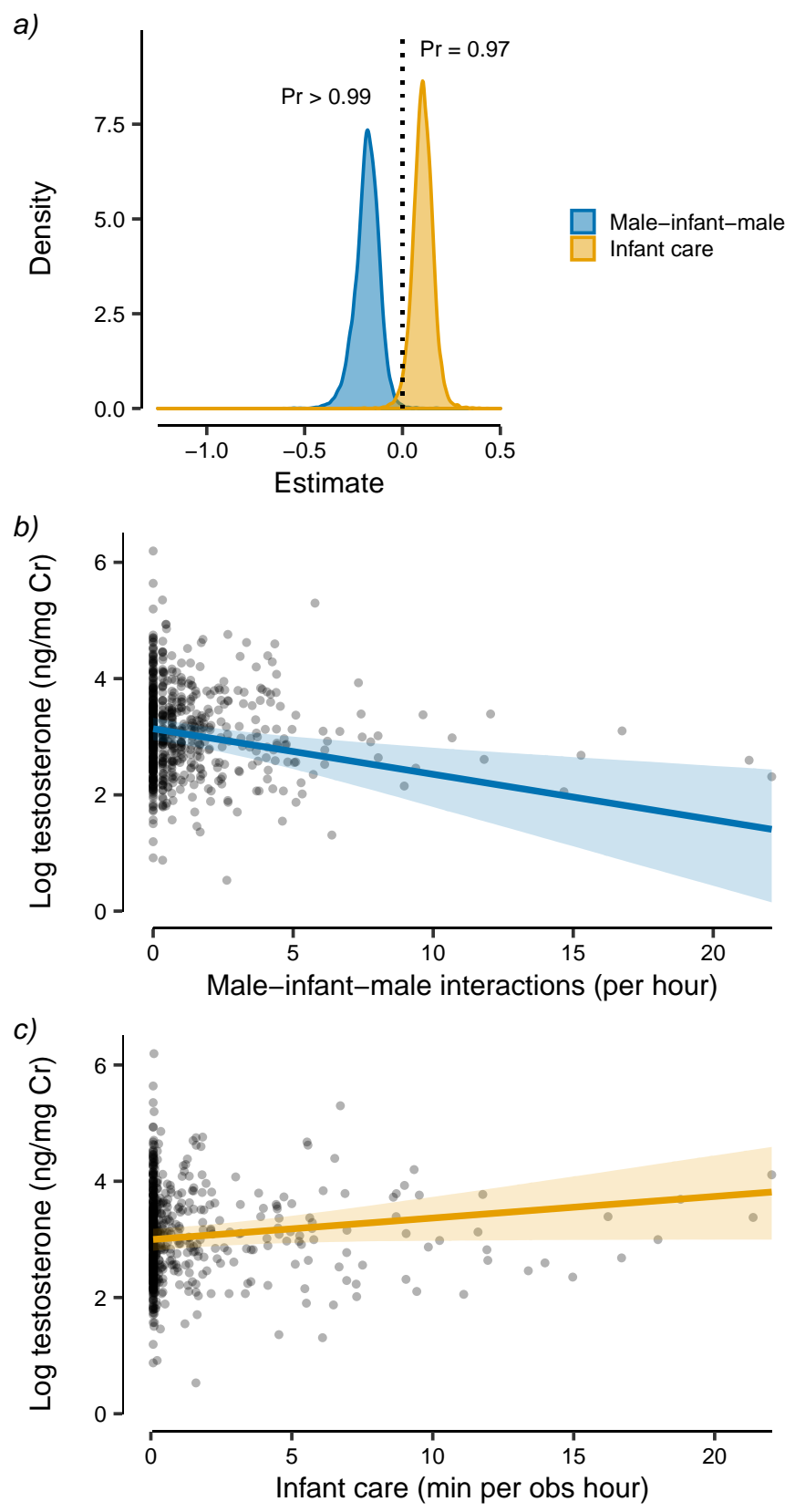

Figure 4.1: Urinary testosterone levels in relation to male-infant-male interactions and infant care (model 1). a) Posterior probability distribution of the slope estimate. $\mathrm{Pr}=$ proportion of the posterior samples that fall on the same side of 0 as the mean. b) and c) Model fitted values (solid line) and $95 \%$ credible intervals (shaded area) when all other predictors are at their mean. $\mathrm{Cr}=$ creatinine. Circles indicate raw data points (urine samples). Note that the fitted model line is based on model results with additional controls that are not reflected in the raw data points. 
a)
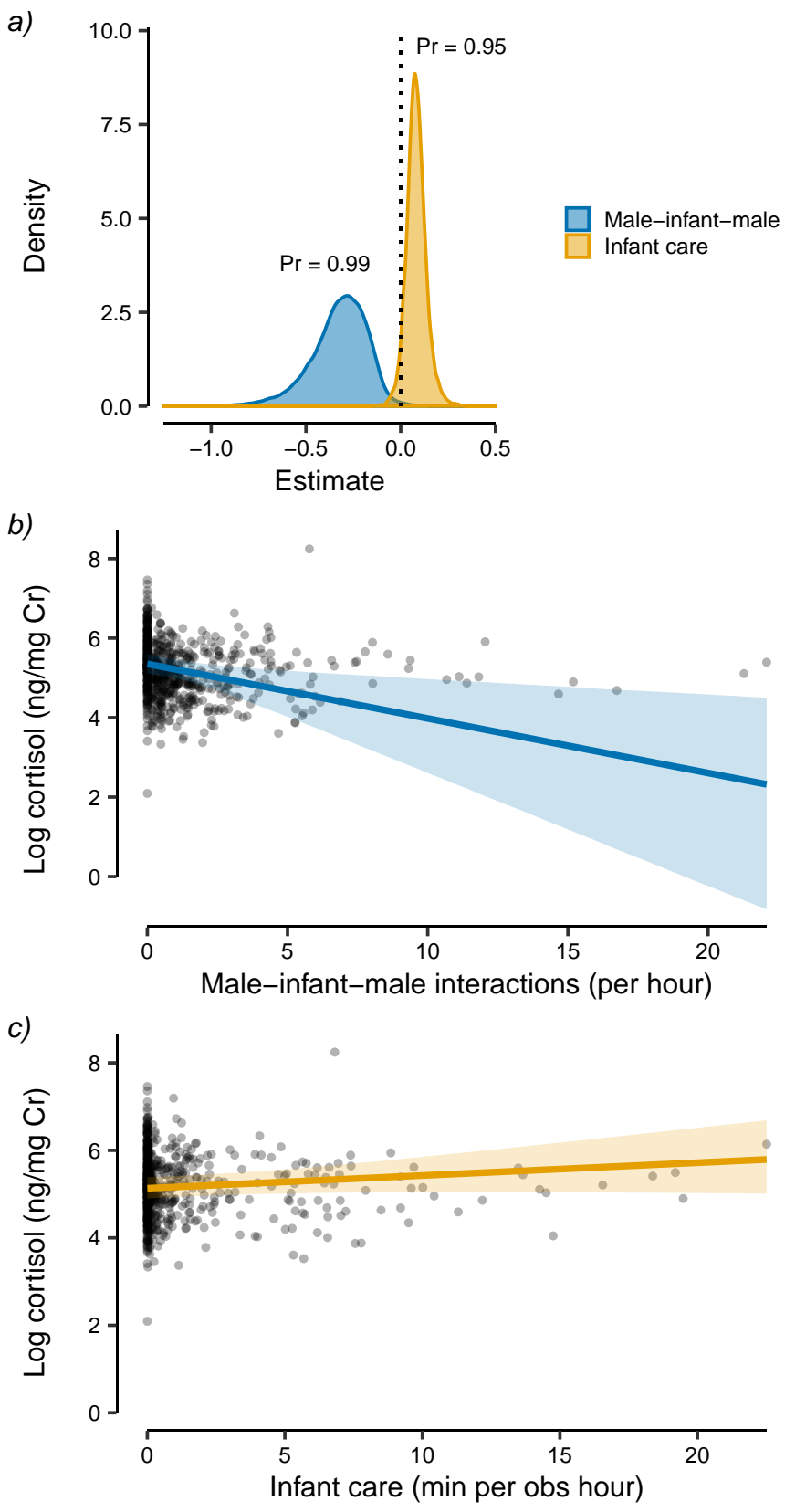

Figure 4.2: Urinary cortisol levels in relation to male-infant-male interactions and infant care (model 2). a) Posterior probability distribution of the slope estimate. $\operatorname{Pr}=$ proportion of the posterior samples that fall on the same side of 0 as the mean. b) and c) Model fitted values (solid line) and $95 \%$ credible intervals (shaded area) when all other predictors are at their mean. $\mathrm{Cr}=$ creatinine. Circles indicate raw data points (urine samples). Note that the fitted model line is based on model results with additional controls that are not reflected in the raw data points. 
social bonds (S/P theory: van Anders et al., 2011). As predicted, urinary testosterone and cortisol levels were negatively correlated with triadic male-infant-male interactions, a ritualized behavior hypothesized to promote and/or maintain social bonds in male macaques (Berghänel et al., 2011a; Kalbitz et al., 2017; Paul et al., 1996). We also investigated whether male care for infants was negatively associated with testosterone (van Anders et al., 2011) and positively associated with cortisol levels (Henkel et al., 2010). Our predictions were partially supported as both testosterone and cortisol levels were positively correlated with male infant care, suggesting that infant care may be done under competitive, rather that nurturing contexts.

Our finding that urinary testosterone levels were negatively correlated with male-infant-male interactions in Barbary macaques supports predictions of the $\mathrm{S} / \mathrm{P}$ theory that low testosterone levels are conducive to nurturing relationships and bonding (van Anders, 2013; van Anders et al., 2011). These results are also in line with previous studies in humans and chimpanzees, which found that behaviors promoting bond formation between same-sex adults are associated with lowered testosterone levels (Ketay et al., 2017; Sobolewski et al., 2012). Same-sex adults may see each other as reproductive rivals and as such, high testosterone levels may interfere with the bonding process by cognitively priming individuals to be competitive rather than nurturing (Boksem et al., 2013; Eisenegger et al., 2011). Human, chimpanzee and macaque males readily form close social bonds and long-term cooperative relationships of adaptive significance (Aktipis et al., 2018; Schülke et al., 2010; Watts, 2002; Young et al., 2013a), and may have been under selective pressure to down-regulate testosterone during periods of affiliation to facilitate bond formation. It would be interesting to test whether lowered testosterone after affiliation between same-sex dyads is only found in species where long-term cooperative relationships increase fitness. Beyond the formation of same-sex social bonds, high testosterone levels may also be detrimental to the maintenance of these bonds in the long term. For example, territory holder male manakins (Pipra filicauda), a lekking bird species, must perform cooperative display with floater males to attract females, but territory holders whose testosterone levels are too high fail to maintain stable display partnerships with floater males (Ryder et al., 2019), reducing their ability to compete for females.

We additionally found that urinary cortisol levels were negatively correlated with rates of male-infant-male interactions in male Barbary macaques. One explanation for this finding is that male-infant-male interactions decrease tensions between adult males, as per the agonistic buffering hypothesis (Deag \& Crook, 1971; Paul et al., 1996), and consequently reduce cortisol levels. Another non-mutually exclusive explanation relates to the potentially bond-formation properties of male-infant-male interactions, resulting in integration into the male social network (Henkel et al., 2010) and increased social support (Berghänel et al., 2011a). Social support is a powerful regulator of the HPA axis and thus of glucocorticoid levels (Hostinar et al., 2014). Merely the presence of closely bonded conspecifics during a stressor is enough to buffer HPA axis activity in a variety of species (Hostinar et al., 2014). Indeed, male Barbary macaques with strong social bonds show attenuated GC responses to social and environmental stressors (Young et al., 2014a). Across primates, subordinate individuals exhibit higher GC levels than dominants only in societies in which they are harassed by dominants but lack access to social 
support (Abbott et al., 2003). Having reliable social support may also help to downregulate HPA activity even in the immediate absence of stressors (Rosal et al., 2004; Wittig et al., 2016), possibly due to increased psychological wellbeing (Massen et al., 2010). Thus, on days when the study males engaged in male-infant-male interactions most frequently, they may have perceived themselves as having strengthened their social bonds and ability to call on support if needed (Berghänel et al., 2011a), consequently lowering cortisol.

The process of bonding itself is likely driven by hormones other than testosterone and cortisol. For instance, the S/P theory predicts that high oxytocin levels are needed to promote social bonding (van Anders et al., 2011). In a previous study on the same group of Barbary macaques we did not find an increase in oxytocin levels following male-infant-male interactions in general but found that oxytocin levels were only higher after male-infant-male interactions with non-bond partners (Rincon et al., 2019a). If it is the combination of low testosterone and high oxytocin that is responsible for the bonding effects, then male-infant-male interactions may function to form bonds selectively between non-bonded partners while physiologically not affecting existing bonds between bond partners. In this interpretation, male-infant-male interactions are a tool for bond formation rather than bond maintenance, although, low testosterone levels after interactions with bond partners may still be beneficial to avoid damaging existing relationships. In our study subjects, $45 \%$ of male-infant-male interactions occurred with non-bond partners (Rincon et al., unpublished data) indicating that there is substantial variation in partner identity and thus the potential to bond with previously non-bonded partners is high. Finally, the low levels of testosterone associated with male-infant-male interactions occur independently of infant care, suggesting that it is not merely the presence of an infant that lowers testosterone or cortisol levels but that the male-infant-male interaction itself is what is salient.

Low cortisol in conjunction with low testosterone may act synergistically to reduce tension and facilitate bonding. During a dyadic friendship formation task in humans, participants desired to be closer to their partners if they (their partner) had low cortisol levels (Ketay et al., 2017), suggesting that affiliative interactions in a relaxed state are beneficial for both partners. While low testosterone and low cortisol may be beneficial to bond formation between same-sex adults, this pattern may not generalize to other types of dyads or contexts. Our findings are in contrast to a previous study in humans where testosterone levels were negatively related to friendship formation and positively related to friendship maintenance within a social network, whereas the opposite was true for cortisol levels, suggesting that testosterone and cortisol have opposite effects with regard to friendship formation and maintenance (Kornienko et al., 2016). One difference between our study and Kornienko et al. (2016) is that the social network comprised a mixed sex group. Steroid dynamics with relation to opposite-sex bonding may differ from that for same-sex bonding due to opposite-sex dyads having greater potential for a sexual relationship and same-sex dyads having greater potential to be reproductive competitors. For example, high testosterone levels promote men to affiliate with women and facilitate the initial stage of sexual or romantic relationships (Roney \& Gettler, 2015). Although high testosterone levels may be detrimental to satisfaction in long-term romantic relationships (Roney \& Gettler, 2015), it is 
unclear if this is also the case for long-term opposite-sex friendships.

Contrary to our predictions, male infant care was positively correlated with testosterone levels. The same was true for cortisol levels, corroborating a previous finding in this species (Henkel et al., 2010). These results suggest that, from the male's perspective, infant care is neither nurturing nor relaxing and may instead be done under a competitive context. Infant caretakers have a protective role in the majority of Old World nonhuman primates (Muller, 2017). The threat of infanticide has been shown to elicit positive testosterone and GC responses in primates (Cheney et al., 2015; Muller, 2017). Infanticide is rare in Barbary macaques and most likely reflects effective counter measures employed by females to induce paternity confusion (e.g. reproductive synchrony: Young et al., 2013a). Nevertheless, males do prefer to interact with infants based on their past mating success with the mother (Kubenova et al., 2019b), which may be indicative of paternal investment and serve as motivation to defend or support an infant against conspecifics. Infant care during the non-mating season also predicts future copulations with the mother in the following mating season in Barbary macaques (Kubenova et al., 2019b). Thus, male infant care in Barbary macaques could also be considered as mating effort. Whether infant care is done as a protective role or as part of mating effort, a positive relationship with testosterone and cortisol could be beneficial in both cases.

Another motivation for males to care for infants may be to engage in affiliative contact with other males (Henkel et al., 2010; Paul et al., 1996) with the ultimate purpose of strengthening male bonds, forging alliances and increasing/maintaining status. For instance, infant-holders are more likely to initiate male-infant-male interactions with males who are higher ranking or adjacent in rank to themselves (Kubenova et al., 2019a; Paul et al., 1996) - males that would constitute the best coalitionary allies. Therefore, infants are attractive commodities and could create competition among males to gain access to them due to there being only a limited number of infants relative to adult males. Once an infant is in a male's 'possession' they may need to fend off interest from other group members and scout their social environment for potential affiliation opportunities with preferred males. In this scenario, caring for infants could be rather stressful and/or requiring a state of alertness. Moderate increases in testosterone and cortisol during infant care may not only help retain the infant in possession but also promote the use of infants as tools to affiliate with other males. For instance, in humans, testosterone promotes males to behave in such a way to increase/maintain social status even if such behaviors are prosocial in nature (Boksem et al., 2013; Eisenegger et al., 2011). Similarly, moderate increases in GC levels serve to promote seeking of affiliative contact in group-living species with complex social systems (Raulo \& Dantzer, 2018). Therefore, elevated testosterone and cortisol levels during infant care are not necessarily a 'cost' to enter the male network as previously suggested (Henkel et al., 2010), but a beneficial endocrine profile that allows males to better navigate their social environment.

Collectively our results provide support for the $\mathrm{S} / \mathrm{P}$ theory which stipulates that high testosterone levels reflect mating effort and low levels are ideal for nurturing relationships including in same-sex adult bonding. Social behavior and dynamics is a complex process. 
Theoretical frameworks that integrate multiple endocrine systems, such as the $\mathrm{S} / \mathrm{P}$ theory (van Anders et al., 2011), are useful in painting a more complete picture of the social context and ultimate function of particular social interactions.

\section{Acknowledgements}

We thank Ellen Merz and Roland Hilgartner for permission to conduct the study at Affenberg Salem. We thank Lauren Cassidy, Tatjana Kaufmann and Lilah Sciaky for help with urine sample and behavioral data collection. We are grateful to Andrea Heistermann for assistance with hormone analyses. This research was funded by the Deutsche Forschungsgemeinschaft (DFG, German Research Foundation) - Project number 254142454 / GRK 2070. 
CHAPTER FIVE 


\section{Chapter 5}

\section{General Discussion}

\subsection{Summary of results}

By using the steroid/peptide theory of social bonds (S/P theory; van Anders et al. (2011)) as a guide, I investigated multiple endocrine axes in relation to social behavior and contributed to our knowledge of the endocrine systems involved in regulating bonding in male Barbary macaques. The $\mathrm{S} / \mathrm{P}$ theory posits that testosterone mediates a competition-nurturance trade-off and that the ideal physiological conditions for social bonding are high oxytocin coupled with low testosterone. While not explicitly part of the $\mathrm{S} / \mathrm{P}$ theory, cortisol is also highly responsive to social interactions that relate to social integration and bonding (Cacioppo et al., 2015; Hostinar et al., 2014).

In study 1 (chapter 2) I validated the suitability of three enzyme immunoassays (EIA), one for cortisol and two for testosterone, for assessing adrenocortical and gonadal activity, respectively, from urine samples in male Barbary macaques. I compared measurements form EIA to those of liquid chromatography-mass spectrometry (LC-MS), a technique that does not suffer from cross-reactivity problems. Cortisol measurements by EIA correlated significantly with LC-MS in both adult and immature males, when measured from unprocessed (i.e. neat) urine samples. The strong correlation between these measurements suggests that the cortisol EIA was not significantly affected by cross-reactivity with other metabolites and is suitable to assess adrenocortical activity in both adult and immature males. Urinary testosterone had to be enzymatically hydrolyzed before EIA measurements correlated significantly with LC-MS in adult males. However, enzymatic hydrolysis did not improve EIA measurements in immature males. Furthermore, we compared testosterone levels of adult males to those of immature males as a biological validation. Testosterone levels were significantly higher in adult males than immature males as measured by LC-MS but not by either testosterone EIA using hydrolyzed urine. Collectively, these results suggest that both testosterone EIAs are suitable to assess gonadal activity in adult males, but not immature males, and only after an enzymatic hydrolysis has been performed prior to analysis. The cortisol and testosterone EIAs validated in this study were used to measure urinary testosterone and cortisol levels of Barbary macaques in study 3 (chapter 4 ).

In study 2 (chapter 3 ) I investigated the role of oxytocin in bonding between adults outside of a pair bond context, with an aim to establish whether oxytocin release was partner specific. As predicted, oxytocin levels were elevated following grooming interactions with any (adult) partner 
compared to a non-social control period. Due to the low number of grooming interactions with non-bond partners we could not formally test partner specific oxytocin release for this behavior. Contrary to our predictions, oxytocin levels were higher after triadic male-infant-male interactions with non-bond partners rather than bond partners, suggesting that male-infant-male interactions function to form new bonds. Given that we found elevated oxytocin levels following grooming with any partner, we tested whether being in a grooming interaction, and thus putatively elevated oxytocin, would increase the probability to accept a solicitation for support in an agonistic conflict but did not find an effect.

In study 3 (chapter 4) I investigated the relationship of testosterone and cortisol with same-sex (male-male) bonding and male infant care, testing predictions of the $\mathrm{S} / \mathrm{P}$ theory. As predicted, male-infant-male interactions were negatively correlated with both testosterone and cortisol suggesting that low levels are beneficial during bonding interactions between same-sex adults. By contrast, male infant care was positively related to both testosterone and cortisol, possibly reflecting a protective role of infant care and/or that infants are attractive commodities, where access to is competed for, and used as a tool to increase or maintain status in this species.

\subsection{Application of non-invasive methods in behavioral ecology}

The validation of immunoassays to assess steroid concentrations in non-invasively collected samples is critical to ensure reliable and biologically valid measurements. The need to validate steroid immunoassays for new species and/or sample matrix due to differences in steroid metabolism across species and excreta type, remains an underappreciated concern despite numerous authors highlighting this issue (Behringer \& Deschner, 2017; Palme, 2019; Touma \& Palme, 2005). For example, over one third of published studies measuring fecal GC metabolites have used methods not adequately validated for the study species (Palme, 2019). Importantly, in chapter 2 I demonstrated that even within the same species, both testosterone EIAs tested produced valid for measurements in one age class (adult males) but not in the other (immature males). Similar discrepancies have been found in testosterone immunoassays validated for adult males that do not produce reliable results in adult females (Goymann, 2005; Preis et al., 2011). Testosterone immunoassays are particularly prone to confounding cross-reactivity problems as the absolute concentration of testosterone is generally lower than similarly structured steroids of adrenal (rather than gonadal) origin such as cortisol and DHEA (Hauser et al., 2008a). Thus, even if an antibody has low cross-reactivity with other steroids, the sheer concentration of adrenal steroids relative to testosterone makes it more likely that results will be confounded. As demonstrated in chapter 2, one way to mitigate the impact of cross-reacting metabolites is to increase the concentration of the target hormone in the sample via deconjugation and extraction of the steroids.

The use of LC-MS to validate immunoassay measurements is beneficial as it helps researchers 
validate EIAs in new species their excreta and offers an additional method to conduct a validation entirely non-invasively. Thus, LC-MS is more practical to conduct validations in wild animals than invasive alternatives. LC-MS is particularly powerful in combination with a biological validation because it ensures that the measurements can capture biologically meaningful variation in hormone levels. Nevertheless, traditional validation methods (e.g. radiometabolism study) will remain invaluable as the information that they provide cannot be replaced by measurements via LC-MS. Radiometabolism studies are particularly useful as they are able to assess excretion pathway of hormones (i.e. proportion of hormone excreted in urine or feces) and time lag to excretion in excreta. Such information is necessary for time-matching hormone levels to social and environmental events that are crucial in the study of behavioral endocrinology.

Validation of methods to quantify hormones from non-invasively collected samples has paved the way for an explosion of wildlife endocrinology studies (Higham, 2016; Palme, 2019). A key benefit of these methods is that it allows researchers to study the expression of hormones in relation to behavior within the socially and ecologically valid settings that these behaviors evolved in. Matching hormone concentrations to animal behavior within these contexts reveals the endocrinological underpinnings of reproduction, competition and dominance relationships, social stressors, and social bonding (Behringer \& Deschner, 2017; Palme, 2019). Not only are non-invasive methods useful for studying social relationships but also in many other areas including female reproductive state, nutritional balance, animal welfare, conservation, and even biomedicine (Behringer \& Deschner, 2017; Palme, 2019). The higher practicality of these methods facilitates the studies of more species than previously possible. Comparisons of hormone-behavior interactions across species of different social systems will ultimately reveal the links between current adaptations and the evolutionary pressures driving their evolution.

\subsection{Oxytocin and social bonding in non-reproductive contexts}

Building on studies indicating oxytocin's crucial role in mother-offspring attachment and pair bonds (Numan \& Young, 2016; Ross \& Young, 2009), researchers have begun exploring if oxytocin is involved in bonding between adults outside of a pair-bond context. Major characteristics of strong social bonds involve frequent affiliative interactions and mutual cooperation/social support (Dunbar \& Shultz, 2010; Massen et al., 2010; Ostner \& Schülke, 2018). The exact underlying mechanisms that motivate individuals to build and maintain such relationships, however, remains unknown. Early theories suggesting that animals cooperate or exchange affiliations by calculated reciprocity (Trivers, 1971) are now deemed unlikely as such situations are rare in nature (Cheney, 2011; Clutton-Brock, 2009). The exchange of affiliations between bonded partners is usually balanced over the long-term but short-term imbalances are often tolerated (Cheney, 2011; Schino \& Aureli, 2009). A more likely explanation are that these relationships are mediated by emotions (i.e. emotional bookkeeping: Schino \& Aureli, 2009; see 
also de Waal, 2000). It is possible that oxytocin release after affiliative interactions induces positive emotions through its interaction with the reward system (Dölen et al., 2013; Skuse \& Gallagher, 2009). Furthermore, oxytocin release may also make the interaction more salient (Shamay-Tsoory \& Abu-Akel, 2016), personal, and strengthen the memory of it (D'Cunha et al., 2011; Ferguson et al., 2000; Numan \& Young, 2016), thus further reinforcing recall of a positive experience and increasing the likelihood that positive interactions will reoccur in the future. Determining the naturally occurring contexts or partners which trigger oxytocin release is helpful in elucidating the dynamics of bond formation and maintenance in group living animals.

Studies investigating non-sexual triggers of oxytocin release under natural conditions have produced mixed results regarding the partner specificity of release. Some studies have suggested that oxytocin release preferentially occurs with bond partners (Crockford et al., 2013; Finkenwirth et al., 2015), while others have suggested that oxytocin release occurs independently of partner strength (i.e. with bond and non-bond partners) (Preis et al., 2018; Samuni et al., 2018b; Wittig et al., 2014). In chapter 3 I found that oxytocin was higher following grooming with any partner. I was not able to determine if release was partner specific with regards to grooming in male Barbary macaques, as most hormone samples were collected after grooming with bond partners. Nevertheless, this result is consistent with previous studies that found elevated oxytocin following grooming with bond partner in chimpanzees (Crockford et al., 2013), and following grooming with any partner in tufted capuchins (Benítez et al., 2018). With regards to male-infant-male interactions, my results were contrary to previous studies that suggest it is affiliation with bond partners, rather than non-bond partners, that trigger oxytocin release (Crockford et al., 2013; Finkenwirth et al., 2015). Instead, I found that oxytocin levels were only elevated after interactions with non-bond partners and not with bond partners. If oxytocin indeed mediates social bonds through emotional bookkeeping (Schino \& Aureli, 2009), then the partner specificity of oxytocin release could be a factor in deciding whether a particular behavior serves as bond maintenance versus bond formation. For instance, if oxytocin is only released after interactions with bond partners (e.g. Crockford et al., 2013), then only those interactions become associated with positive emotions and thus the existing bond is reinforced (i.e. maintenance). If oxytocin is only released after interactions with non-bond partners, as with male-infant-male interactions in male Barbary macaques (chapter 3), then those interactions become associated with positive emotions and thus a new bond is potentially created (i.e. formation). If oxytocin release is independent of bond strength (e.g. Wittig et al., 2014), then oxytocin potentially facilitates bond maintenance and formation. Clearly though, more replication studies are needed to determine how consistent these findings are as even within the same species results are mixed (Crockford et al., 2013; Preis et al., 2018).

Due to the correlational nature of chapter 3 , it is possible that rather than being released in response to affiliative interactions, oxytocin levels were elevated prior to interaction and promoted the interaction to happen. For instance, oxytocin has anxiolytic effects and can promote approach towards, while simultaneously reducing the tendency to avoid, conspecifics (Kemp \& Guastella, 2011). Under this view, in chapter 3 oxytocin promoted adult males 
to approach any partner (male or female irrespective of bond strength) to groom, but only non-bonded males for male-infant-male interactions. There are two possible reasons for this. Most grooming occurred with adult females, whereas all male-infant-male interactions occurred (by definition) with other adult males. The situation between adult males are generally tense and is reflected by the fact that affiliative contact between them usually happens in the presence of an infant, which likely acts as a buffer (Deag \& Crook, 1971; Paul et al., 1996). This tension may be especially high between non-bonded male partners as their interactions may be more unpredictable than with bond partners. While males were always motivated to partake in male-infant-male interactions with a bond partner, they were only motivated to interact with non-bond partners if their oxytocin levels were elevated. With regards to grooming, most grooming occurred with females and apparently males were only motivated to groom females if their oxytocin levels were high. Oxytocin's effect on promoting approach and reducing avoidance behaviors is not mutually exclusive with its effect in promoting bonding once they affiliate. It could be that oxytocin both promotes grooming, and is released by grooming, and promotes bonding once released. However, the lack of elevated oxytocin following male-infant-male interactions with bond partners suggests that oxytocin is not released in this particular context. As our sample size for interactions with non-bond partners was small, it is prudent to refrain from drawing strong conclusions about a partner-specific effect with this behavior.

Some authors have argued that the role of oxytocin in bonding in Old World primates differs to that of most other mammalian taxa due to their relatively large brains (Dunbar, 2010; Machin \& Dunbar, 2011). It is argued that oxytocin may simply facilitate social engagement and perhaps bond formation, but that the maintenance of social bonds is under the control of brain opioids, particularly endorphins (Dunbar, 2010; Machin \& Dunbar, 2011). Considering that we did not find an effect on male-infant-male interactions with bond partner on oxytocin, it is possible that endorphins may play a role in the bonding outcomes of this behavior. For example, male-infant-male interactions are ritualistic and partially synchronized in facial signals (Deag, 1980; Deag \& Crook, 1971). At least in humans, performing synchronized behaviors is positively correlated with endorphin levels and feelings of closeness (Dunbar \& Shultz, 2010; Tarr et al., 2015, 2016). Thus, brain endorphin release may be a mechanism by which male-infant-male interactions are able to maintain existing social bonds in male Barbary macaques. In practice, however, it is difficult to disentangle the unique contributions of oxytocin and endorphins since most behaviors that trigger the release of oxytocin also trigger the release of endorphins (e.g. grooming in primates: Keverne et al., 1989; Benítez et al., 2018; Crockford et al., 2013), and both interact with the dopaminergic system (Devine et al., 1993; Peciña et al., 2015; Skuse \& Gallagher, 2009).

Finally, oxytocin may further mediate social relationships by promoting cooperation during polyadic between-group conflicts as shown for humans and chimpanzees (De Dreu, 2012; Samuni et al., 2017; Ten Velden et al., 2017). I investigated whether naturally elevated oxytocin would promote cooperation in a within-group polyadic conflict as a secondary analysis in chapter 3 . I did not find that putatively elevated oxytocin following a grooming interaction increased the probability to accept a solicitation for help from another adult during a conflict. In fact, there 
were only in a few cases where the grooming partner was the one who solicited help. The low frequency of these events may explain the lack of effect, as nonhuman primates are more likely to help a caller if they were recently groomed by them (Cheney et al., 2010; Hemelrijk, 1994). Clearly then, oxytocin did not universally increase the probability to cooperate with any partner in my study. This is perhaps not surprising as oxytocin has previously been found to only promote cooperation in specific contexts or with specific partners (Bartz et al., 2011). However, I cannot rule out the possibility that oxytocin would increase the likelihood of cooperating with bond partners in within-group conflicts, which seems to be the most likely scenario and is a question best addressed by long-term field studies.

\subsection{Oxytocin, testosterone, cortisol, and same-sex social bonds}

The primary behavior of interest across chapter 3 and chapter 4 was triadic male-infant-male interactions - a ritualized male bonding behavior (Berghänel et al., 2011a; Deag, 1980; Kalbitz et al., 2017). The physiological profile for this behavior was positive with oxytocin after interactions with non-bond partners but not bond partners, and negative with testosterone and cortisol after interacting with any partner. The $\mathrm{S} / \mathrm{P}$ theory predicts that the ideal physiological conditions for bonding are high oxytocin coupled with low testosterone. As discussed above, oxytocin is likely the primary hormone driving the bonding process. As oxytocin was only elevated after male-infant-male interactions with non-bond partners, this suggests that these interactions serve to form new bonds while physiologically not affecting existing bonds. Additionally, there are several reasons why low testosterone and cortisol levels may be associated with bonding behaviors, particularly between same-sex adults.

To my knowledge, there are no studies that have investigated same-sex social bonding or affiliative behavior in relation to oxytocin, testosterone, and cortisol altogether. There are, however, studies that have looked at different paired combinations of these hormones and their overall picture is in line with my findings about male-infant-male interactions. For instance, male chimpanzees have high oxytocin (Samuni et al., 2018b; Wittig et al., 2014) and low testosterone (Sobolewski et al., 2012) levels following food-sharing events, a behavior implicated in bond formation (Mitani \& Watts, 2001; Wittig et al., 2014). High oxytocin occurred after food sharing between both mixed- and same-sex dyads (Samuni et al., 2018b; Wittig et al., 2014) and low testosterone after food sharing between same-sex dyads (male-male) (Sobolewski et al., 2012). Similarly, levels of testosterone and cortisol decline following a friendship formation task designed to increase feelings of closeness between same-sex human dyads (Ketay et al., 2017). Low testosterone levels consistently occur following same-sex bonding behaviors across studies on Barbary macaques (chapter 4), chimpanzees, and humans (Ketay et al., 2017; Sobolewski et al., 2012), and are consistent with the S/P theory prediction that testosterone mediates a trade-off between competition and nurturing and bonding (van Anders, 2013; van Anders et al., 2011). High testosterone may interfere with the bonding process by affecting cognitive 
processes that are beneficial to bonding (Eisenegger et al., 2011). For example, testosterone has an inhibitory role in trust (Boksem et al., 2013; Bos et al., 2010), empathy (Ronay \& Carney, 2013; van Honk et al., 2011), and facial mimicry (Hermans et al., 2006). Same-sex adults in particular could perceive each other as reproductive rivals and high testosterone could prime individuals to be competitive rather than nurturing. Macaques, chimpanzees, and humans are all known to form strong same-sex bonds and long-term cooperative relationships, which are known to increased fitness (Aktipis et al., 2018; Schülke et al., 2010; Watts, 2002; Young et al., 2013a). Individuals may thus have been under selective pressure to inhibit testosterone excretion during same-sex bonding periods, providing a ripe environment for bond formation.

As with testosterone and same-sex bonding, the social buffering effects of HPA activity due to perceived social support are most likely rooted in fitness benefits that the partner provides (Kiyokawa \& Hennessy, 2018). Social buffering may be partner and context specific, where the ideal buffering partner may change across the life span. For example, mothers provide the most effective buffering effects to their infants who are dependent on them for survival during this period of their lives (Gunnar \& Hostinar, 2015; Kiyokawa \& Hennessy, 2018). Species of pair bonded animals find their pair-mate to be the best partner for buffering stress as they are the most relevant for reproductive success (Gunnar \& Hostinar, 2015; Kiyokawa \& Hennessy, 2018). In the context of non-sexual bonds, female chacma baboons (Papio ursinus) with a dependent infant show buffered GC responses when a potentially infanticidal male migrates into the group if they have a male friend (Beehner et al., 2005). Presumably these buffering effects are driven by male bond partners (as opposed to females) because they are able to successfully defend the mother's infant from infanticidal males, and consequently their infants are at lower risk of infanticide (Beehner et al., 2005). For adult male Barbary macaques, other adult males also provide the most effective and contextually relevant agonistic support and strongly bonded males are known to buffer HPA axis response for each other against aggression received (Young et al., 2014a). Additionally, Barbary macaques are aware of the strength of social bonds that other group members have with each other (Kubenova et al., 2017), such that the threat of coalitionary support is powerful enough to destabilize dyadic dominance relationships in males (Berghänel et al., 2011b). Therefore, the social buffering effects that male social bond strength has (Young et al., 2014a) may be due to males perceiving that they can count coalitionary support more often from bonded male partners during conflicts (Young et al., 2014b). Male-infant-male interactions predict coalition formation (Berghänel et al., 2011a), likely through bond formation, making this behavior clearly salient to males, so much so that it buffers cortisol levels even in the absence of stressors (chapter 4). Grooming, on the other hand, is comparatively rare between adult males (Paul et al., 1996), not correlated to fecal GC levels (Henkel et al., 2010), and is therefore perhaps less important for integrating males for coalitionary purposes in this species.

Due to the correlational nature of chapter 4, it is not possible to ascertain the directionality of the hormone-behavior interactions. It is possible that male-infant-male interactions lowered steroid levels, or that low steroid levels promoted male-infant-male interactions, or both. In the case of testosterone, either explanation would still be in line with the $\mathrm{S} / \mathrm{P}$ theory that predicts low testosterone levels are ideal for bonding. In the case of cortisol, I would argue that it is less likely 
that lower levels were motivated individuals to engage in male-infant-male interactions. I make this argument because moderate increases in GCs generally have a positive effect on motivating individuals to seek affiliative contact in species with complex social systems (Dantzer et al., 2014). This propensity to affiliate could occur because high GCs may be signaling a lack of adequate social bonding and thus serve as motivation to fix this (e.g. Engh et al., 2006). Thus, a more likely explanation is that it is the perceived strengthening of bonds that lowered GC levels. However, an experimental design would be needed to confirm if this is indeed the case in male Barbary macaques.

\subsection{Interaction between HPG and HPA axes in competitive contexts}

Testosterone and GCs are often co-released or interact in behavioral contexts outside of bonding. For example, these two steroids are often studied in conjunction in the context of social challenges (Casto \& Edwards, 2016; Deviche et al., 2014; Gesquiere et al., 2011; Grebe et al., 2019; Higham et al., 2013; Jaeggi et al., 2018; Landys et al., 2007; Oyegbile \& Marler, 2006; Salvador, 2012). It is easy to foresee why these two hormones might be beneficial during competition. Testosterone cognitively primes individuals for competition (Oliveira, 2004), while cortisol increase alertness and mobilize energy for potentially demanding encounters (Sapolsky, 2002).

In chapter 4, I found that infant care in male Barbary macaques was positively correlated with both testosterone and cortisol levels. Although the primary care behaviors (i.e. huddling, carrying, grooming) appear to be nurturing, a positive relationship with testosterone and cortisol suggests that male infant care is primarily undertaken under competitive contexts in this species. Paternal care takes a protective role in most Old World primate species (Muller, 2017), whose function likely evolved due to high infanticide risk (Paul et al., 2000). Elevated testosterone and cortisol are often exhibited by males whose infants are at risk of infanticide (Cheney et al., 2015; Muller, 2017; Ostner et al., 2008), probably as an adaptation to this threat. Barbary macaque males engage in infanticide rarely, likely counteracted by female strategies to confuse paternity such as post-conception copulations (Young et al., 2013b), promiscuity (Small, 1990), and reproductive synchrony (Young et al., 2013a). Regardless, mating success with females is known to predict which infants males prefer to interact with (Kubenova et al., 2019b). These preferences may indicate paternal investment and facilitate protective interactions (i.e. defend, support) in favor of an infant against conspecifics. Simultaneously, male infant care in this species could indicate mating effort as this behavior predicts future mating opportunities with its mother in the next mating season (Kubenova et al., 2019b; Ménard et al., 2001), and thus a positive relationship with testosterone would be expected.

While mutually elevated testosterone and cortisol seem beneficial in some competitive situations, the HPG and HPA axes are able to functionally interact by suppressing the activity of the other via their major hormonal outputs, androgens and GCs, respectively (Terburg et al., 2009; Viau, 
2002). The dual hormone hypothesis (Mehta \& Josephs, 2010; Mehta \& Prasad, 2015) was the first to formalize that the action of testosterone in dominance-related behavior in humans depends on cortisol levels. This hypothesis proposes that the effects of testosterone on status only occur when cortisol levels are low (Mehta \& Josephs, 2010; Mehta \& Prasad, 2015). In other words, high cortisol levels block the status-seeking effects of testosterone. Since its conception, support for the dual hormone hypothesis is mixed (Grebe et al., 2019). A recent meta-analysis found marginal support for this hypothesis, highlighting the need for better definitions of what status seeking means, and under what contexts, before progress can be made (Dekkers et al., 2019). The dual hormone hypothesis is yet to be formally tested in nonhuman animals. In nonhuman primates, the relationship between rank with testosterone and GCs is mixed and appears to depend on the social style of the species (Abbott et al., 2003; Muller, 2017). A more consistent relationship emerges when dominance hierarchies are unstable as generally both hormones are elevated (Gesquiere et al., 2011; Higham et al., 2013; Setchell et al., 2008, 2010), likely as a reflection of increased competition and stress during these periods.

Although I did not aim to formally test the dual hormone hypothesis, one motivation that Barbary macaque males have to handle infants - aside from a protective role - is to use them as tools to facilitate affiliative contact with other males (Henkel et al., 2010; Paul et al., 1996). If the ultimate purpose of infant handling is to build cooperative alliances and increase/maintain rank, then infant care could potentially be classified as "status-seeking". In this case, we would expect high testosterone but low cortisol levels to occur during infant handling, as per the dual hormone hypothesis (Mehta \& Josephs, 2010; Mehta \& Prasad, 2015). This relationship was not what I found, as both testosterone and cortisol were elevated (chapter 4), and conflicts with the dual hormone hypothesis. However, there remains a possibility that not all infant care in Barbary macaques are equal. Some males could care for infants in a protective role, whereas others may use them as tools. If such a distinction exists, it would be interesting to test if these two different functions exhibit different physiological profiles. Here I would predict that males who infant handle for defensive purposes exhibit elevated testosterone and cortisol; in contrast, I would expect those seeking higher status during these interactions to have high testosterone but low cortisol.

\subsection{Conclusions and outlook}

Using the S/P theory (van Anders et al., 2011) as a framework, I set out to investigate the endocrinological mechanisms underlying social bonds between adults outside a pair bond context, while additionally investigating the HPA axis where appropriate. In line with the $\mathrm{S} / \mathrm{P}$ theory, behaviors related to bonding - grooming and male-infant-male interactions - were associated with elevated oxytocin levels. Male-infant-male interactions associated with oxytocin release were partner specific, and only elevated following interactions with non-bond partners but not bond partners. This specificity suggests that male-infant-male interactions function to form new social bonds rather than maintain existing ones. Furthermore, I found that male-infant-male 
interactions were negatively correlated with testosterone and cortisol, suggesting that these two hormones may be beneficial during bond formation between same-sex partners. Altogether these results suggest that while oxytocin may be driving the bonding process, low levels of testosterone and cortisol may act to facilitate and/or not inhibit this process. The S/P theory may additionally be useful to help classify contexts where behaviors are performed where they may be ambiguous (van Anders et al., 2011). For example, I was able to classify infant care provided by adult male Barbary macaques as a competitive rather than a nurturing behavior, given the positive relationship that this behavior had with testosterone and cortisol.

The oxytocinergic system, HPG, and HPA axes likely act in concert to produce behavioral and cognitive responses that are adaptive to the situation, including priming individuals to be nurturing rather than competitive during bonding periods. These systems and their homologues are highly conserved across vertebrates (Goodson, 2013; Soares et al., 2010). Yet there is a wide diversity in the degree of sociality, bonding and cooperation across species that these very same systems support (Goodson, 2013; Ostner \& Schülke, 2014). While the oxytocinergic system is highly conserved across mammals, the exact social contexts in which its release is triggered and the types of bonds that it promotes, varies greatly even within closely related species (Goodson, 2013). Finding bonding mechanisms in one species (e.g. voles) does not necessarily give predictive accuracy for other species (Goodson, 2013). Theoretical frameworks that are able to integrate multiple endocrine systems, such as the S/P theory (van Anders et al., 2011) and others (e.g. Mehta \& Prasad, 2015; Soares et al., 2010), may increase our predictive accuracy about what types of bonds or relationship will develop after a social interaction given a hormonal profile that it produces. Future studies should investigate the dynamics of bond formation and maintenance from a longitudinal perspective and test whether a certain "bonding" endocrine profile is predictive of future bond maintenance and formation within individuals. For example, in the case of Barbary macaques, do male-infant-male interactions where both males repeatedly exhibit elevated oxytocin coupled with low testosterone and cortisol, reliably result in bond formation and cooperation in the near to long-term future? Conversely, do dyads that fail to exhibit this endocrine profile also fail to form bonds and develop a cooperative relationship? Is oxytocin release after affiliation always/never partner specific? Answering these questions will further our understanding of the dynamics of bond formation and elucidate how important is the role of hormones in bonding relative to cognition (Broad et al., 2006; Curley \& Keverne, 2005).

Finally, perhaps research on the oxytocinergic system in relation social behavior, could borrow from concepts developed for the HPG (Wingfield et al., 1990) and HPA (Romero et al., 2009) axes. The challenge hypothesis introduced the concept of hormone state levels, where homeostatic functions, sexual behaviors and coping with challenges, each require different state levels of testosterone (Wingfield et al., 2019, 1990). Curiously, oxytocin levels in chimpanzees following bonding related behaviors such as grooming and food-sharing (Crockford et al., 2013; Wittig et al., 2014) were significantly lower than those found after inter-group conflicts, which require coordinated aggression against an out-group (Samuni et al., 2017). Could oxytocin also operate on qualitatively different state levels relating to bonding and cooperation? As research 
on the natural triggers of oxytocin release during bonding and cooperation continues to be conducted in more species, such patterns may begin to emerge. 
REFERENCES 


\section{References}

Abbott, D. H., Keverne, E. B., Bercovitch, F. B., Shively, C. A., Mendoza, S. P., Saltzman, W., Snowdon, C. T., Ziegler, T. E., Banjevic, M., Garland, T., \& Sapolsky, R. M. (2003). Are subordinates always stressed? A comparative analysis of rank differences in cortisol levels among primates. Hormones and Behavior, 43(1), 67-82. https://doi.org/10.1016/S0018-506X(02) 00037-5

Adkins-Regan, E. (2005). Hormones and Animal Social Behavior. Princeton University Press.

Aktipis, A., Cronk, L., Alcock, J., Ayers, J. D., Baciu, C., Balliet, D., Boddy, A. M., Curry, O. S., Krems, J. A., Muñoz, A., Sullivan, D., Sznycer, D., Wilkinson, G. S., \& Winfrey, P. (2018). Understanding cooperation through fitness interdependence. Nature Human Behaviour, 2(7), 429-431. https://doi.org/10.1038/s41562-018-0378-4

Al-Dujaili, E. A. S. (2006). Development and validation of a simple and direct ELISA method for the determination of conjugated (glucuronide) and non-conjugated testosterone excretion in urine. Clinica Chimica Acta, 364(1), 172-179. https://doi.org/10.1016/j.cccn.2005.06.019

Amico, J. A., Challinor, S. M., \& Cameron, J. L. (1990). Pattern of oxytocin concentrations in the plasma and cerebrospinal fluid of lactating rhesus monkeys (Macaca mulatta): Evidence for functionally independent oxytocinergic pathways in primates. The Journal of Clinical Endocrinology \& Metabolism, 71 (6), 1531-1535. https://doi.org/10.1210/jcem-71-6-1531

Amico, J. A., Ulbrecht, J. S., \& Robinson, A. G. (1987). Clearance studies of oxytocin in humans using radioimmunoassay measurements of the hormone in plasma and urine. The Journal of Clinical Endocrinology \& Metabolism, 64(2), 340-345. https://doi.org/10.1210/jcem-64-2-340

Archer, J. (2006). Testosterone and human aggression: An evaluation of the challenge hypothesis. Neuroscience \& Biobehavioral Reviews, 30(3), 319-345. https://doi.org/10.1016/j. neubiorev.2004.12.007

Archie, E. A., Tung, J., Clark, M., Altmann, J., \& Alberts, S. C. (2014). Social affiliation matters: Both same-sex and opposite-sex relationships predict survival in wild female baboons. Proceedings of the Royal Society B, 281(1793), 20141261. https://doi.org/10.1098/rspb.2014. 1261

Aronsen, G. P., Beuerlein, M. M., Watts, D. P., \& Bribiescas, R. G. (2015). Redtail and red colobus monkeys show intersite urinary cortisol concentration variation in Kibale National Park, Uganda. Conservation Physiology, 3(1). https://doi.org/10.1093/conphys/cov006

Arueti, M., Perach-Barzilay, N., Tsoory, M. M., Berger, B., Getter, N., \& Shamay-Tsoory, S. 
G. (2013). When two become one: The role of oxytocin in interpersonal coordination and cooperation. Journal of Cognitive Neuroscience, 25(9), 1418-1427. https://doi.org/10.1162/ jocn_a_00400

Bahr, N. I., Palme, R., Möhle, U., Hodges, J. K., \& Heistermann, M. (2000). Comparative aspects of the metabolism and excretion of cortisol in three individual nonhuman primates. General and Comparative Endocrinology, 117(3), 427-438. https://doi.org/10.1006/gcen.1999. 7431

Baid, S. K., Sinaii, N., Wade, M., Rubino, D., \& Nieman, L. K. (2007). Radioimmunoassay and tandem mass spectrometry measurement of bedtime salivary cortisol levels: A comparison of assays to establish hypercortisolism. The Journal of Clinical Endocrinology $\mathcal{E}$ Metabolism, 92 (8), 3102-3107. https://doi.org/10.1210/jc.2006-2861

Barr, D. J., Levy, R., Scheepers, C., \& Tily, H. J. (2013). Random effects structure for confirmatory hypothesis testing: Keep it maximal. Journal of Memory and Language, 68(3), 255-278. https://doi.org/10.1016/j.jml.2012.11.001

Bartz, J. A., Zaki, J., Bolger, N., \& Ochsner, K. N. (2011). Social effects of oxytocin in humans: Context and person matter. Trends in Cognitive Sciences, 15(7), 301-309. https: //doi.org/10.1016/j.tics.2011.05.002

Beehner, J. C., Bergman, T. J., Cheney, D. L., Seyfarth, R. M., \& Whitten, P. L. (2005). The effect of new alpha males on female stress in free-ranging baboons. Animal Behaviour, 69(5), 1211-1221. https://doi.org/10.1016/j.anbehav.2004.08.014

Behringer, V., \& Deschner, T. (2017). Non-invasive monitoring of physiological markers in primates. Hormones and Behavior, 91, 3-18. https://doi.org/10.1016/j.yhbeh.2017.02.001

Behringer, V., Hohmann, G., Stevens, J. M. G., Weltring, A., \& Deschner, T. (2012). Adrenarche in bonobos (Pan paniscus): Evidence from ontogenetic changes in urinary dehydroepiandrosterone-sulfate levels. Journal of Endocrinology, 214(1), 55-65. https: //doi.org/10.1530/JOE-12-0103

Benítez, M. E., Sosnowski, M. J., Tomeo, O. B., \& Brosnan, S. F. (2018). Urinary oxytocin in capuchin monkeys: Validation and the influence of social behavior. American Journal of Primatology, 80(10), e22877. https://doi.org/10.1002/ajp.22877

Berghänel, A., Ostner, J., Schröder, U., \& Schülke, O. (2011a). Social bonds predict future cooperation in male Barbary macaques, Macaca sylvanus. Animal Behaviour, 81(6), 1109-1116. https://doi.org/10.1016/j.anbehav.2011.02.009

Berghänel, A., Ostner, J., \& Schülke, O. (2011b). Coalitions destabilize dyadic dominance 
relationships in male Barbary macaques (Macaca Sylvanus). Behaviour, 148(11-13), 1256-1274. https://doi.org/10.1163/000579511X600628

Boksem, M. A. S., Mehta, P. H., Van den Bergh, B., van Son, V., Trautmann, S. T., Roelofs, K., Smidts, A., \& Sanfey, A. G. (2013). Testosterone inhibits trust but promotes reciprocity. Psychological Science, 24 (11), 2306-2314. https://doi.org/10.1177/0956797613495063

Bos, P. A., Terburg, D., \& Honk, J. van. (2010). Testosterone decreases trust in socially naïve humans. Proceedings of the National Academy of Sciences, 107(22), 9991-9995. https: //doi.org/10.1073/pnas.0911700107

Brent, L. J. N., Semple, S., Dubuc, C., Heistermann, M., \& MacLarnon, A. (2011). Social capital and physiological stress levels in free-ranging adult female rhesus macaques. Physiology \& Behavior, 102(1), 76-83. https://doi.org/10.1016/j.physbeh.2010.09.022

Broad, K. D., Curley, J. P., \& Keverne, E. B. (2006). Mother-infant bonding and the evolution of mammalian social relationships. Philosophical Transactions of the Royal Society B: Biological Sciences, 361 (1476), 2199-2214. https://doi.org/10.1098/rstb.2006.1940

Brown, J. L., Kersey, D. C., Freeman, E. W., \& Wagener, T. (2010). Assessment of diurnal urinary cortisol excretion in Asian and African elephants using different endocrine methods. Zoo Biology, 29(2), 274-283. https://doi.org/10.1002/zoo.20268

Brown, J. L., Wasser, S. K., Wildt, D. E., \& Graham, L. H. (1994). Comparative aspects of steroid hormone metabolism and ovarian activity in felids, measured noninvasively in feces. Biology of Reproduction, 51 (4), 776-786. https://doi.org/10.1095/biolreprod51.4.776

Buchanan, K. L., \& Goldsmith, A. R. (2004). Noninvasive endocrine data for behavioural studies: The importance of validation. Animal Behaviour, 67(1), 183-185.

Bürkner, P.-C. (2017). Brms: An R package for Bayesian multilevel models using Stan. Journal of Statistical Software, 80(1), 1-28. https://doi.org/10.18637/jss.v080.i01

Cacioppo, J. T., Cacioppo, S., Capitanio, J. P., \& Cole, S. W. (2015). The neuroendocrinology of social isolation. Annual Review of Psychology, 66(1), 733-767. https://doi.org/10.1146/ annurev-psych-010814-015240

Cameron, E. Z., Setsaas, T. H., \& Linklater, W. L. (2009). Social bonds between unrelated females increase reproductive success in feral horses. Proceedings of the National Academy of Sciences, 106 (33), 13850-13853. https://doi.org/10.1073/pnas.0900639106

Campbell, L. A. D., Tkaczynski, P. J., Lehmann, J., Mouna, M., \& Majolo, B. (2018). Social thermoregulation as a potential mechanism linking sociality and fitness: Barbary macaques 
with more social partners form larger huddles. Scientific Reports, 8(1), 6074. https://doi.org/ 10.1038/s41598-018-24373-4

Carter, G. G., \& Wilkinson, G. S. (2015). Intranasal oxytocin increases social grooming and food sharing in the common vampire bat Desmodus rotundus. Hormones and Behavior, 75, 150-153. https://doi.org/10.1016/j.yhbeh.2015.10.006

Casto, K. V., \& Edwards, D. A. (2016). Testosterone, cortisol, and human competition. Hormones and Behavior, 82, 21-37. https://doi.org/10.1016/j.yhbeh.2016.04.004

Chang, S. W. C., Barter, J. W., Ebitz, R. B., Watson, K. K., \& Platt, M. L. (2012). Inhaled oxytocin amplifies both vicarious reinforcement and self reinforcement in rhesus macaques (Macaca mulatta). Proceedings of the National Academy of Sciences, 109(3), 959-964. https: //doi.org/10.1073/pnas.1114621109

Cheney, D. L. (2011). Extent and limits of cooperation in animals. Proceedings of the National Academy of Sciences, 108 (Supplement 2), 10902-10909. https://doi.org/10.1073/ pnas. 1100291108

Cheney, D. L., Crockford, C., Engh, A. L., Wittig, R. M., \& Seyfarth, R. M. (2015). The costs of parental and mating effort for male baboons. Behavioral Ecology and Sociobiology, 69(2), 303-312. https://doi.org/10.1007/s00265-014-1843-3

Cheney, D. L., Moscovice, L. R., Heesen, M., Mundry, R., \& Seyfarth, R. M. (2010). Contingent cooperation between wild female baboons. Proceedings of the National Academy of Sciences, 107(21), 9562-9566. https://doi.org/10.1073/pnas.1001862107

Cheney, D. L., \& Seyfarth, R. M. (2009). Stress and coping mechanisms in female primates. In Advances in the Study of Behavior (Vol. 39, pp. 1-44). Academic Press. https://doi.org/10. 1016/S0065-3454(09)39001-4

Clutton-Brock, T. (2009). Cooperation between non-kin in animal societies. Nature, 462(7269), 51-57. https://doi.org/10.1038/nature08366

Cohen, S., \& Wills, T. A. (1985). Stress, social support, and the buffering hypothesis. Psychological Bulletin, 98(2), 310-357. https://doi.org/10.1037/0033-2909.98.2.310

Crockford, C., Deschner, T., \& Wittig, R. M. (2017). The role of oxytocin in social buffering: What do primate studies add? In R. Hurlemann \& V. Grinevich (Eds.), Behavioral Pharmacology of Neuropeptides: Oxytocin (Vol. 35, pp. 155-173). Cham: Springer. https://doi.org/10.1007/7854_2017_12

Crockford, C., Deschner, T., Ziegler, T. E., \& Wittig, R. M. (2014). Endogenous peripheral 
oxytocin measures can give insight into the dynamics of social relationships: A review. Frontiers in Behavioral Neuroscience, 8. https://doi.org/10.3389/fnbeh.2014.00068

Crockford, C., Wittig, R. M., Langergraber, K., Ziegler, T. E., Zuberbühler, K., \& Deschner, T. (2013). Urinary oxytocin and social bonding in related and unrelated wild chimpanzees. Proceedings of the Royal Society B, 280(1755), 20122765. https://doi.org/10.1098/rspb.2012. 2765

Cross, T. G., \& Hornshaw, M. P. (2016). Can LC and LC-MS ever replace immunoassays? Journal of Applied Bioanalysis, 2(4), 108-116. https://doi.org/10.17145/jab

Curley, J. P., \& Keverne, E. B. (2005). Genes, brains and mammalian social bonds. Trends in Ecology \& Evolution, 20(10), 561-567. https://doi.org/10.1016/j.tree.2005.05.018

Danish, L. M., Heistermann, M., Agil, M., \& Engelhardt, A. (2015). Validation of a novel collection device for non-invasive urine sampling from free-ranging animals. PLoS ONE, 10(11), e0142051. https://doi.org/10.1371/journal.pone.0142051

Dantzer, B., Fletcher, Q. E., Boonstra, R., \& Sheriff, M. J. (2014). Measures of physiological stress: A transparent or opaque window into the status, management and conservation of species? Conservation Physiology, 2(1). https://doi.org/10.1093/conphys/cou023

D'Cunha, T. M., King, S. J., Fleming, A. S., \& Lévy, F. (2011). Oxytocin receptors in the nucleus accumbens shell are involved in the consolidation of maternal memory in postpartum rats. Hormones and Behavior, 59(1), 14-21. https://doi.org/10.1016/j.yhbeh.2010.09.007

Deag, J. M. (1980). Interactions between males and unweaned Barbary macaques: Testing the agonistic buffering hypothesis. Behaviour, 75(1-2), 54-80. https://doi.org/10.1163/ 156853980X00564

Deag, J. M., \& Crook, J. H. (1971). Social behaviour and "agonistic buffering" in the wild Barbary macaque Macaca sylvana L. Folia Primatologica, 15(3-4), 183-200. https://doi.org/ $10.1159 / 000155378$

Declerck, C. H., Boone, C., \& Kiyonari, T. (2010). Oxytocin and cooperation under conditions of uncertainty: The modulating role of incentives and social information. Hormones and Behavior, 57(3), 368-374. https://doi.org/10.1016/j.yhbeh.2010.01.006

De Dreu, C. K. W. (2012). Oxytocin modulates cooperation within and competition between groups: An integrative review and research agenda. Hormones and Behavior, 61 (3), 419-428. https://doi.org/10.1016/j.yhbeh.2011.12.009

De Dreu, C. K. W., Greer, L. L., Handgraaf, M. J. J., Shalvi, S., Kleef, G. A. V., Baas, M., 
Velden, F. S. T., Dijk, E. V., \& Feith, S. W. W. (2010). The neuropeptide oxytocin regulates parochial altruism in intergroup conflict among humans. Science, 328(5984), 1408-1411. https: //doi.org/10.1126/science.1189047

Dekkers, T. J., van Rentergem, J. A. A., Meijer, B., Popma, A., Wagemaker, E., \& Huizenga, H. M. (2019). A meta-analytical evaluation of the dual-hormone hypothesis: Does cortisol moderate the relationship between testosterone and status, dominance, risk taking, aggression, and psychopathy? Neuroscience \& Biobehavioral Reviews, 96, 250-271. https://doi.org/10. 1016/j.neubiorev.2018.12.004

de Turckheim, G., \& Merz, E. (1984). Breeding Barbary macaques in outdoor open enclosures. In J. E. Fa (Ed.), The Barbary macaque: A case study in conservation (pp. 241-261). Boston, MA: Springer US. https://doi.org/10.1007/978-1-4613-2785-1_10

Deviche, P., Beouche-Helias, B., Davies, S., Gao, S., Lane, S., \& Valle, S. (2014). Regulation of plasma testosterone, corticosterone, and metabolites in response to stress, reproductive stage, and social challenges in a desert male songbird. General and Comparative Endocrinology, 203, 120-131. https://doi.org/10.1016/j.ygcen.2014.01.010

Devine, D. P., Leone, P., Pocock, D., \& Wise, R. A. (1993). Differential involvement of ventral tegmental $\mathrm{mu}$, delta and kappa opioid receptors in modulation of basal mesolimbic dopamine release: In vivo microdialysis studies. Journal of Pharmacology and Experimental Therapeutics, $266(3), 1236-1246$.

de Vries, H., Stevens, J. M. G., \& Vervaecke, H. (2006). Measuring and testing the steepness of dominance hierarchies. Animal Behaviour, 71(3), 585-592. https://doi.org/10.1016/j.anbehav. 2005.05.015

de Waal, F. B. M. (2000). Attitudinal reciprocity in food sharing among brown capuchin monkeys. Animal Behaviour, 60(2), 253-261. https://doi.org/10.1006/anbe.2000.1471

Dölen, G., Darvishzadeh, A., Huang, K. W., \& Malenka, R. C. (2013). Social reward requires coordinated activity of nucleus accumbens oxytocin and serotonin. Nature, 501(7466), 179-184. https://doi.org/10.1038/nature12518

Dunbar, R. I. M. (2010). The social role of touch in humans and primates: Behavioural function and neurobiological mechanisms. Neuroscience 83 Biobehavioral Reviews, 34 (2), 260-268. https: //doi.org/10.1016/j.neubiorev.2008.07.001

Dunbar, R., \& Shultz, S. (2010). Bondedness and sociality. Behaviour, 147(7), 775-803. https: //doi.org/10.1163/000579510X501151

Eastman, S.-a. K., Makawiti, D. W., Collins, W. P., \& Hodges, J. K. (1984). Pattern of excretion 
of urinary steroid metabolites during the ovarian cycle and pregnancy in the marmoset monkey. Journal of Endocrinology, 102(1), 19-26. https://doi.org/10.1677/joe.0.1020019

Edelstein, R. S., van Anders, S. M., Chopik, W. J., Goldey, K. L., \& Wardecker, B. M. (2014). Dyadic associations between testosterone and relationship quality in couples. Hormones and Behavior, 65 (4), 401-407. https://doi.org/10.1016/j.yhbeh.2014.03.003

Eisenegger, C., Haushofer, J., \& Fehr, E. (2011). The role of testosterone in social interaction. Trends in Cognitive Sciences, 15(6), 263-271. https://doi.org/10.1016/j.tics.2011.04.008

Engh, A. L., Beehner, J. C., Bergman, T. J., Whitten, P. L., Hoffmeier, R. R., Seyfarth, R. M., \& Cheney, D. L. (2006). Behavioural and hormonal responses to predation in female chacma baboons (Papio hamadryas ursinus). Proceedings of the Royal Society B, 273(1587), 707-712. https://doi.org/10.1098/rspb.2005.3378

Ferguson, J. N., Young, L. J., Hearn, E. F., Matzuk, M. M., Insel, T. R., \& Winslow, J. T. (2000). Social amnesia in mice lacking the oxytocin gene. Nature Genetics, 25(3), 284-288. https://doi.org/10.1038/77040

Fieß, M., Heistermann, M., \& Hodges, J. K. (1999). Patterns of urinary and fecal steroid excretion during the ovarian cycle and pregnancy in the African elephant (Loxodonta africana). General and Comparative Endocrinology, 115(1), 76-89. https://doi.org/10.1006/gcen.1999. 7287

Finkenwirth, C., Martins, E., Deschner, T., \& Burkart, J. M. (2016). Oxytocin is associated with infant-care behavior and motivation in cooperatively breeding marmoset monkeys. Hormones and Behavior, 80, 10-18. https://doi.org/10.1016/j.yhbeh.2016.01.008

Finkenwirth, C., van Schaik, C., Ziegler, T. E., \& Burkart, J. M. (2015). Strongly bonded family members in common marmosets show synchronized fluctuations in oxytocin. Physiology \& Behavior, 151, 246-251. https://doi.org/10.1016/j.physbeh.2015.07.034

Fooden, J. (2007). Systematic review of the Barbary macaque, Macaca sylvanus (Linnaeus, 1758). Fieldiana Zoology, 2007(113), 1-60. https://doi.org/dzf3p6

Francis, S. M., Kirkpatrick, M. G., de Wit, H., \& Jacob, S. (2016). Urinary and plasma oxytocin changes in response to MDMA or intranasal oxytocin administration. Psychoneuroendocrinology, 74, 92-100. https://doi.org/10.1016/j.psyneuen.2016.08.011

French, J. A., Cavanaugh, J., Mustoe, A. C., Carp, S. B., \& Womack, S. L. (2018). Social monogamy in nonhuman primates: Phylogeny, phenotype, and physiology. The Journal of Sex Research, 55 (4-5), 410-434. https://doi.org/10.1080/00224499.2017.1339774 
Frère, C. H., Krützen, M., Mann, J., Connor, R. C., Bejder, L., \& Sherwin, W. B. (2010). Social and genetic interactions drive fitness variation in a free-living dolphin population. Proceedings of the National Academy of Sciences, 107(46), 19949-19954. https://doi.org/10.1073/pnas. 1007997107

Fürtbauer, I., Heistermann, M., Schülke, O., \& Ostner, J. (2014). Low female stress hormone levels are predicted by same- or opposite-sex sociality depending on season in wild Assamese macaques. Psychoneuroendocrinology, 48, 19-28. https://doi.org/10.1016/j.psyneuen.2014.05. 022

Ganswindt, A., Palme, R., Heistermann, M., Borragan, S., \& Hodges, J. K. (2003). Non-invasive assessment of adrenocortical function in the male African elephant (Loxodonta africana) and its relation to musth. General and Comparative Endocrinology, 134(2), 156-166. https://doi.org/ 10.1016/S0016-6480(03)00251-X

Gesquiere, L. R., Learn, N. H., Simao, M. C. M., Onyango, P. O., Alberts, S. C., \& Altmann, J. (2011). Life at the top: Rank and stress in wild male baboons. Science, 333(6040), 357-360. https://doi.org/10.1126/science.1207120

Gesquiere, L. R., Ziegler, T. E., Chen, P. A., Epstein, K. A., Alberts, S. C., \& Altmann, J. (2014). Measuring fecal testosterone in females and fecal estrogens in males: Comparison of RIA and LC/MS/MS methods for wild baboons (Papio Cynocephalus). General and Comparative Endocrinology, 204, 141-149. https://doi.org/10.1016/j.ygcen.2014.04.021

Gettler, L. T., McDade, T. W., Feranil, A. B., \& Kuzawa, C. W. (2011). Longitudinal evidence that fatherhood decreases testosterone in human males. Proceedings of the National Academy of Sciences, 108 (39), 16194. https://doi.org/10.1073/pnas.1105403108

Giles, L. C., Glonek, G. F. V., Luszcz, M. A., \& Andrews, G. R. (2005). Effect of social networks on 10 year survival in very old Australians: The Australian longitudinal study of aging. Journal of Epidemiology \& Community Health, 59(7), 574-579. https://doi.org/10. $1136 /$ jech.2004.025429

Gimpl, G., \& Fahrenholz, F. (2001). The oxytocin receptor system: Structure, function, and regulation. Physiological Reviews, 81(2), 629-683. https://doi.org/10.1152/physrev.2001.81.2. 629

Goodson, J. L. (2013). Deconstructing sociality, social evolution and relevant nonapeptide functions. Psychoneuroendocrinology, 38(4), 465-478. https://doi.org/10.1016/j.psyneuen. 2012.12.005

Goymann, W. (2005). Noninvasive monitoring of hormones in bird droppings: Physiological validation, sampling, extraction, sex differences, and the influence of diet on hormone metabolite 
levels. Annals of the New York Academy of Sciences, 1046(1), 35-53. https://doi.org/10.1196/ annals. 1343.005

Goymann, W., \& Flores, D. P. (2017). Acute peaks of testosterone suppress paternal care: Evidence from individual hormonal reaction norms. Proceedings of the Royal Society B, 284(1857), 20170632. https://doi.org/10.1098/rspb.2017.0632

Goymann, W., Moore, I. T., \& Oliveira, R. F. (2019). Challenge hypothesis 2.0: A fresh look at an established idea. BioScience, 69(6), 432-442. https://doi.org/10.1093/biosci/biz041

Grange, R. D., Thompson, J. P., \& Lambert, D. G. (2014). Radioimmunoassay, enzyme and non-enzyme-based immunoassays. British Journal of Anaesthesia, 112(2), 213-216. https: //doi.org/10.1093/bja/aet293

Gray, P. B., Chapman, J. F., Burnham, T. C., McIntyre, M. H., Lipson, S. F., \& Ellison, P. T. (2004). Human male pair bonding and testosterone. Human Nature, 15(2), 119-131. https://doi.org/10.1007/s12110-004-1016-6

Grebe, N. M., Del Giudice, M., Emery Thompson, M., Nickels, N., Ponzi, D., Zilioli, S., Maestripieri, D., \& Gangestad, S. W. (2019). Testosterone, cortisol, and status-striving personality features: A review and empirical evaluation of the dual hormone hypothesis. Hormones and Behavior, 109, 25-37. https://doi.org/10.1016/j.yhbeh.2019.01.006

Gunnar, M. R., \& Hostinar, C. E. (2015). The social buffering of the hypothalamic pituitary adrenocortical axis in humans: Developmental and experiential determinants. Social Neuroscience, 10(5), 479-488. https://doi.org/10.1080/17470919.2015.1070747

Habumuremyi, S., Robbins, M. M., Fawcett, K. A., \& Deschner, T. (2014). Monitoring ovarian cycle activity via progestagens in urine and feces of female mountain gorillas: A comparison of EIA and LC-MS measurements. American Journal of Primatology, 76(2), 180-191. https: //doi.org/10.1002/ajp.22220

Harrison, N., C. Lopes, P., \& König, B. (2017). Oxytocin administration during early pair formation delays communal nursing in female house mice. Animal Behaviour, 123, 61-68. https: //doi.org/10.1016/j.anbehav.2016.10.030

Haunhorst, C. B., Heesen, M., Ostner, J., \& Schülke, O. (2017). Social bonds with males lower the costs of competition for wild female Assamese macaques. Animal Behaviour, 125, 51-60. https://doi.org/10.1016/j.anbehav.2017.01.008

Haunhorst, C. B., Schülke, O., \& Ostner, J. (2016). Opposite-sex social bonding in wild Assamese macaques. American Journal of Primatology, 78(8), 872-882. https://doi.org/10. 1002/ajp.22554 
Hauser, B., Deschner, T., \& Boesch, C. (2008a). Development of a liquid chromatography-tandem mass spectrometry method for the determination of 23 endogenous steroids in small quantities of primate urine. Journal of Chromatography B, 862(1), 100-112. https: //doi.org/10.1016/j.jchromb.2007.11.009

Hauser, B., Schulz, D., Boesch, C., \& Deschner, T. (2008b). Measuring urinary testosterone levels of the great apes - Problems with enzymatic hydrolysis using Helix pomatia juice. General and Comparative Endocrinology, 158(1), 77-86. https://doi.org/10.1016/j.ygcen.2008.05.006

Heistermann, M., \& Hodges, J. K. (1995). Endocrine monitoring of the ovarian cycle and pregnancy in the saddle-back tamarin (Saguinus fuscicollis) by measurement of steroid conjugates in urine. American Journal of Primatology, 35(2), 117-127. https: //doi.org/10.1002/ajp.1350350204

Heistermann, M., Palme, R., \& Ganswindt, A. (2006). Comparison of different enzymeimmunoassays for assessment of adrenocortical activity in primates based on fecal analysis. American Journal of Primatology, 68(3), 257-273. https://doi.org/10.1002/ajp.20222

Hemelrijk, C. K. (1994). Support for being groomed in long-tailed macaques, Macaca fascicularis. Animal Behaviour, 48(2), 479-481. https://doi.org/10.1006/anbe.1994.1264

Henkel, S., Heistermann, M., \& Fischer, J. (2010). Infants as costly social tools in male Barbary macaque networks. Animal Behaviour, 79(6), 1199-1204. https://doi.org/10.1016/j.anbehav. 2010.02 .005

Hennessy, M. B., Kaiser, S., \& Sachser, N. (2009). Social buffering of the stress response: Diversity, mechanisms, and functions. Frontiers in Neuroendocrinology, 30(4), 470-482. https: //doi.org/10.1016/j.yfrne.2009.06.001

Hermans, E. J., Putman, P., \& van Honk, J. (2006). Testosterone administration reduces empathetic behavior: A facial mimicry study. Psychoneuroendocrinology, 31(7), 859-866. https: //doi.org/10.1016/j.psyneuen.2006.04.002

Higham, J. P. (2016). Field endocrinology of nonhuman primates: Past, present, and future. Hormones and Behavior, 84, 145-155. https://doi.org/10.1016/j.yhbeh.2016.07.001

Higham, J. P., Heistermann, M., \& Maestripieri, D. (2013). The endocrinology of male rhesus macaque social and reproductive status: A test of the challenge and social stress hypotheses. Behavioral Ecology and Sociobiology, 67(1), 19-30. https://doi.org/10.1007/s00265-012-1420-6

Hill, R. A., Bentley, R. A., \& Dunbar, R. I. M. (2008). Network scaling reveals consistent fractal pattern in hierarchical mammalian societies. Biology Letters, 4(6), 748-751. https: //doi.org/10.1098/rsbl.2008.0393 
Hinde, R. A. (1976). Interactions, relationships and social structure. Man, 11(1), 1-17. https: //doi.org/10.2307/2800384

Hirschenhauser, K., Kotrschal, K., \& Möstl, E. (2005). Synthesis of measuring steroid metabolites in goose feces. Annals of the New York Academy of Sciences, 1046(1), 138-153. https://doi.org/10.1196/annals.1343.011

Hirschenhauser, K., \& Oliveira, R. F. (2006). Social modulation of androgens in male vertebrates: Meta-analyses of the challenge hypothesis. Animal Behaviour, 71 (2), 265-277. https://doi.org/10.1016/j.anbehav.2005.04.014

Holt-Lunstad, J., Birmingham, W. C., \& Light, K. C. (2014). Relationship quality and oxytocin: Influence of stable and modifiable aspects of relationships. Journal of Social and Personal Relationships. https://doi.org/10.1177/0265407514536294

Holt-Lunstad, J., Smith, T. B., \& Layton, J. B. (2010). Social relationships and mortality risk: A meta-analytic review. PLoS Medicine, 7(7), e1000316. https://doi.org/10.1371/journal. pmed.1000316

Hostinar, C. E., Sullivan, R. M., \& Gunnar, M. R. (2014). Psychobiological mechanisms underlying the social buffering of the HPA axis: A review of animal models and human studies across development. Psychological Bulletin, 140(1). https://doi.org/10.1037/a0032671

Hsing, A. W., Stanczyk, F. Z., Bélanger, A., Schroeder, P., Chang, L., Falk, R. T., \& Fears, T. R. (2007). Reproducibility of serum sex steroid assays in men by RIA and mass spectrometry. Cancer Epidemiology and Prevention Biomarkers, 16(5), 1004-1008. https://doi.org/10.1158/ 1055-9965.EPI-06-0792

Jaeggi, A. V., Trumble, B., C., \& Brown, M. (2018). Group-level competition influences urinary steroid hormones among wild red-tailed monkeys, indicating energetic costs. American Journal of Primatology, 80(5), e22757. https://doi.org/10.1002/ajp.22757

Jurek, B., \& Neumann, I. D. (2018). The oxytocin receptor: From intracellular signaling to behavior. Physiological Reviews, 98(3), 1805-1908. https://doi.org/10.1152/physrev.00031. 2017

Kalbitz, J., Ostner, J., \& Schülke, O. (2016). Strong, equitable and long-term social bonds in the dispersing sex in Assamese macaques. Animal Behaviour, 113, 13-22. https://doi.org/10. 1016/j.anbehav.2015.11.005

Kalbitz, J., Schülke, O., \& Ostner, J. (2017). Triadic male-infant-male interaction serves in bond maintenance in male Assamese macaques. PLoS ONE, 12(10), e0183981. https://doi. org/10.1371/journal.pone.0183981 
Kemp, A. H., \& Guastella, A. J. (2011). The role of oxytocin in human affect: A novel hypothesis. Current Directions in Psychological Science, 20(4), 222-231. https://doi.org/10. $1177 / 0963721411417547$

Kendrick, K. M., Keverne, E. B., Baldwin, B. A., \& Sharman, D. F. (1986). Cerebrospinal fluid levels of acetylcholinesterase, monoamines and oxytocin during labour, parturition, vaginocervical stimulation, lamb separation and suckling in sheep. Neuroendocrinology, 44(2), 149-156. https://doi.org/10.1159/000124638

Kersey, D. C., Wildt, D. E., Brown, J. L., Huang, Y., Snyder, R. J., \& Monfort, S. L. (2010). Parallel and seasonal changes in gonadal and adrenal hormones in male giant pandas (Ailuropoda melanoleuca). Journal of Mammalogy, 91(6), 1496-1507. https://doi.org/10.1644/09-MAMMA-404.1

Ketay, S., \& Beck, L. A. (2017). Attachment predicts cortisol response and closeness in dyadic social interaction. Psychoneuroendocrinology, 80, 114-121. https://doi.org/10.1016/j.psyneuen. 2017.03.009

Ketay, S., Welker, K. M., Beck, L. A., Thorson, K. R., \& Slatcher, R. B. (2019). Social anxiety, cortisol, and early-stage friendship. Journal of Social and Personal Relationships, 36(7), 1954-1974. https://doi.org/10.1177/0265407518774915

Ketay, S., Welker, K. M., \& Slatcher, R. B. (2017). The roles of testosterone and cortisol in friendship formation. Psychoneuroendocrinology, 76, 88-96. https://doi.org/10.1016/j. psyneuen.2016.11.022

Keverne, E. B., Martensz, N. D., \& Tuite, B. (1989). Beta-endorphin concentrations in cerebrospinal fluid of monkeys are influenced by grooming relationships. Psychoneuroendocrinology, 14 (1), 155-161. https://doi.org/10.1016/0306-4530(89)90065-6

Kiyokawa, Y., \& Hennessy, M. B. (2018). Comparative studies of social buffering: A consideration of approaches, terminology, and pitfalls. Neuroscience 85 Biobehavioral Reviews, 86, 131-141. https://doi.org/10.1016/j.neubiorev.2017.12.005

Kornienko, O., Schaefer, D. R., Weren, S., Hill, G. W., \& Granger, D. A. (2016). Cortisol and testosterone associations with social network dynamics. Hormones and Behavior, 80, 92-102. https://doi.org/10.1016/j.yhbeh.2016.01.013

Krause, J., \& Ruxton, G. D. (2002). Living in Groups. Oxford, New York: Oxford University Press.

Kretzschmar, P., Gansloßer, U., \& Dehnhard, M. (2004). Relationship between androgens, environmental factors and reproductive behavior in male white rhinoceros (Ceratotherium 
simum simum). Hormones and Behavior, 45(1), 1-9. https://doi.org/10.1016/j.yhbeh.2003. 08.001

Kubenova, B., Konecna, M., Majolo, B., Smilauer, P., Ostner, J., \& Schülke, O. (2017). Triadic awareness predicts partner choice in male-infant-male interactions in Barbary macaques. Animal Cognition, 20(2), 221-232. https://doi.org/10.1007/s10071-016-1041-y

Kubenova, B., Ostner, J., Schülke, O., Majolo, B., Smilauer, P., \& Konecna, M. (2019a). The effect of dominance rank on the distribution of different types of male-infant-male interactions in Barbary macaques Macaca sylvanus. International Journal of Primatology. https://doi.org/ 10.1007/s10764-019-00086-x

Kubenova, B., Ostner, J., Schülke, O., Majolo, B., Smilauer, P., Waterman, J., Tkaczynski, P., \& Konecna, M. (2019b). The male and female perspective in the link between male infant care and mating behaviour in Barbary macaques. Ethology, 0(0), 1-11. https://doi.org/10.1111/ eth. 12948

Kulik, L., Muniz, L., Mundry, R., \& Widdig, A. (2012). Patterns of interventions and the effect of coalitions and sociality on male fitness. Molecular Ecology, 21(3), 699-714. https: //doi.org/10.1111/j.1365-294X.2011.05250.x

Küster, J., \& Paul, A. (1992). Influence of male competition and female mate choice on male mating success in Barbary macaques (Macaca sylvanus). Behaviour, 120(3), 192-216. https: //doi.org/10.1163/156853992X00606

Landgraf, R., \& Neumann, I. D. (2004). Vasopressin and oxytocin release within the brain: A dynamic concept of multiple and variable modes of neuropeptide communication. Frontiers in Neuroendocrinology, 25(3), 150-176. https://doi.org/10.1016/j.yfrne.2004.05.001

Landgraf, R., Neumann, I., \& Schwarzberg, H. (1988). Central and peripheral release of vasopressin and oxytocin in the conscious rat after osmotic stimulation. Brain Research, $457(2)$, 219-225. https://doi.org/10.1016/0006-8993(88)90689-0

Landys, M. M., Goymann, W., Raess, M., \& Slagsvold, T. (2007). Hormonal responses to male-male social challenge in the blue tit cyanistes caeruleus: Single-broodedness as an explanatory variable. Physiological and Biochemical Zoology, 80(2), 228-240. https://doi.org/10.1086/510564

Lemoine, N. P. (2019). Moving beyond noninformative priors: Why and how to choose weakly informative priors in Bayesian analyses. Oikos, 128(7), 912-928. https://doi.org/10.1111/oik. 05985

Leng, G., \& Sabatier, N. (2016). Measuring oxytocin and vasopressin: Bioassays, immunoassays 
and random numbers. Journal of Neuroendocrinology, 28(10). https://doi.org/10.1111/jne. 12413

Lévy, F., Kendrick, K. M., Goode, J. A., Guevara-Guzman, R., \& Keverne, E. B. (1995). Oxytocin and vasopressin release in the olfactory bulb of parturient ewes: Changes with maternal experience and effects on acetylcholine, $\gamma$-aminobutyric acid, glutamate and noradrenaline release. Brain Research, 669(2), 197-206. https://doi.org/10.1016/0006-8993(94)01236-B

Lim, M. M., \& Young, L. J. (2006). Neuropeptidergic regulation of affiliative behavior and social bonding in animals. Hormones and Behavior, 50(4), 506-517. https://doi.org/10.1016/ j.yhbeh.2006.06.028

Love, T. M. (2014). Oxytocin, motivation and the role of dopamine. Pharmacology Biochemistry and Behavior, 119, 49-60. https://doi.org/10.1016/j.pbb.2013.06.011

Lynn, S. E. (2008). Behavioral insensitivity to testosterone: Why and how does testosterone alter paternal and aggressive behavior in some avian species but not others? General and Comparative Endocrinology, 157(3), 233-240. https://doi.org/10.1016/j.ygcen.2008.05.009

Macdonald, K., \& Feifel, D. (2013). Helping oxytocin deliver: Considerations in the development of oxytocin-based therapeutics for brain disorders. Frontiers in Neuroscience, \%. https://doi. org/10.3389/fnins.2013.00035

Machin, A. J., \& Dunbar, R. I. M. (2011). The brain opioid theory of social attachment: A review of the evidence. Behaviour, 148(9-10), 985-1025. https://doi.org/10.1163/ $000579511 X 596624$

Madden, J., \& Clutton-Brock, T. (2011). Experimental peripheral administration of oxytocin elevates a suite of cooperative behaviours in a wild social mammal. Proceedings of the Royal Society B, 278(1709), 1189-1194. https://doi.org/10.1098/rspb.2010.1675

Martin, P., \& Bateson, P. (2007). Measuring behaviour: An introductory guide (3rd ed.). Cambridge: Cambridge University Press.

Massen, J. J. M., \& Sterck, E. H. M. (2013). Stability and durability of intra- and intersex social bonds of captive rhesus macaques (Macaca mulatta). International Journal of Primatology, 34 (4), 770-791. https://doi.org/10.1007/s10764-013-9695-7

Massen, J., Sterck, E., \& Vos, H. de. (2010). Close social associations in animals and humans: Functions and mechanisms of friendship. Behaviour, 147(11), 1379-1412. https://doi.org/10. 1163/000579510X528224

McCallister, J. M., Smith, T. E., \& Elwood, R. W. (2004). Validation of urinary cortisol 
as an indicator of hypothalamic-pituitary-adrenal function in the bearded emperor tamarin (Saguinus imperator subgrisescens). American Journal of Primatology, 63(1), 17-23. https: //doi.org/10.1002/ajp.20033

McCullough, M. E., Churchland, P. S., \& Mendez, A. J. (2013). Problems with measuring peripheral oxytocin: Can the data on oxytocin and human behavior be trusted? Neuroscience E Biobehavioral Reviews, 37(8), 1485-1492. https://doi.org/10.1016/j.neubiorev.2013.04.018

McElreath, R. (2016). Statistical rethinking: A Bayesian course with examples in $R$ and Stan. Chapman and Hall/CRC. https://doi.org/10.1201/9781315372495

McFarland, R., \& Majolo, B. (2013). Coping with the cold: Predictors of survival in wild Barbary macaques, Macaca sylvanus. Biology Letters, 9(4), 20130428. https://doi.org/10. 1098/rsbl.2013.0428

McLeod, P. J., Moger, W. H., Ryon, J., Gadbois, S., \& Fentress, J. C. (1996). The relation between urinary cortisol levels and social behaviour in captive timber wolves. Canadian Journal of Zoology, 74(2), 209-216. https://doi.org/10.1139/z96-026

Mehta, P. H., \& Josephs, R. A. (2010). Testosterone and cortisol jointly regulate dominance: Evidence for a dual-hormone hypothesis. Hormones and Behavior, 58(5), 898-906. https: //doi.org/10.1016/j.yhbeh.2010.08.020

Mehta, P. H., \& Prasad, S. (2015). The dual-hormone hypothesis: A brief review and future research agenda. Current Opinion in Behavioral Sciences, 3, 163-168. https://doi.org/10.1016/ j.cobeha.2015.04.008

Ménard, N., von Segesser, F., Scheffrahn, W., Pastorini, J., Vallet, D., Gaci, B., Martin, R. D., \& Gautier-Hion, A. (2001). Is male-infant caretaking related to paternity and/or mating activities in wild Barbary macaques (Macaca sylvanus)? Comptes Rendus de L'Académie Des Sciences - Series III - Sciences de La Vie, 324(7), 601-610. https://doi.org/10.1016/S07644469(01)01339-7

Mikolajczak, M., Gross, J. J., Lane, A., Corneille, O., de Timary, P., \& Luminet, O. (2010). Oxytocin makes people trusting, not gullible. Psychological Science, 21(8), 1072-1074. https: //doi.org/10.1177/0956797610377343

Mitani, J. C., \& Watts, D. P. (2001). Why do chimpanzees hunt and share meat? Animal Behaviour, 61(5), 915-924. https://doi.org/10.1006/anbe.2000.1681

Moore, I. T., Hernandez, J., \& Goymann, W. (2019). Who rises to the challenge? Testing the challenge hypothesis in fish, amphibians, reptiles, and mammals. Hormones and Behavior. https://doi.org/10.1016/j.yhbeh.2019.06.001 
Moscovice, L. R., Surbeck, M., Fruth, B., Hohmann, G., Jaeggi, A. V., \& Deschner, T. (2019). The cooperative sex: Sexual interactions among female bonobos are linked to increases in oxytocin, proximity and coalitions. Hormones and Behavior, 116, 104581. https://doi.org/ 10.1016/j.yhbeh.2019.104581

Möhle, U., Heistermann, M., Palme, R., \& Hodges, J. K. (2002). Characterization of urinary and fecal metabolites of testosterone and their measurement for assessing gonadal endocrine function in male nonhuman primates. General and Comparative Endocrinology, 129(3), 135-145. https: //doi.org/10.1016/S0016-6480(02)00525-7

Muller, M. N. (2017). Testosterone and reproductive effort in male primates. Hormones and Behavior, 91, 36-51. https://doi.org/10.1016/j.yhbeh.2016.09.001

Muller, M. N., \& Wrangham, R. W. (2004). Dominance, aggression and testosterone in wild chimpanzees: A test of the "challenge hypothesis". Animal Behaviour, 67(1), 113-123. https: //doi.org/10.1016/j.anbehav.2003.03.013

Mundry, R., \& Fischer, J. (1998). Use of statistical programs for nonparametric tests of small samples often leads to incorrect $P$ values: Examples from Animal Behaviour. Animal Behaviour, 56 (1), 256-259. https://doi.org/10.1006/anbe.1998.0756

Müller, N., Heistermann, M., Strube, C., Schülke, O., \& Ostner, J. (2017). Age, but not anthelmintic treatment, is associated with urinary neopterin levels in semi-free ranging Barbary macaques. Scientific Reports, 7, 41973. https://doi.org/10.1038/srep41973

Neumann, I. D. (2002). Involvement of the brain oxytocin system in stress coping: Interactions with the hypothalamo-pituitary-adrenal axis. In D. Poulain, S. Oliet, \& D. Theodosis (Eds.), Progress in Brain Research (Vol. 139, pp. 147-162). Elsevier. https://doi.org/10.1016/S00796123(02)39014-9

Neumann, I. D., \& Landgraf, R. (2012). Balance of brain oxytocin and vasopressin: Implications for anxiety, depression, and social behaviors. Trends in Neurosciences, 35(11), 649-659. https: //doi.org/10.1016/j.tins.2012.08.004

Nowak, R., Keller, M., \& Lévy, F. (2011). Mother-young relationships in sheep: A model for a multidisciplinary approach of the study of attachment in mammals. Journal of Neuroendocrinology, 23(11), 1042-1053. https://doi.org/10.1111/j.1365-2826.2011.02205.x

Numan, M., \& Young, L. J. (2016). Neural mechanisms of mother-infant bonding and pair bonding: Similarities, differences, and broader implications. Hormones and Behavior, 77, 98-112. https://doi.org/10.1016/j.yhbeh.2015.05.015

Oliveira, R. F. (2004). Social modulation of androgens in vertebrates: Mechanisms and function. 
In P. J. B. Slater, J. S. Rosenblatt, C. T. Snowdon, T. J. Roper, H. J. Brockmann, \& M. Naguib (Eds.), Advances in the study of behavior (Vol. 34, pp. 165-239). Academic Press. https://doi.org/10.1016/S0065-3454(04)34005-2

Oliveira, R. F., Hirschenhauser, K., Carneiro, L. A., \& Canario, A. V. M. (2002). Social modulation of androgen levels in male teleost fish. Comparative Biochemistry and Physiology Part B: Biochemistry and Molecular Biology, 132(1), 203-215. https://doi.org/10.1016/S10964959(01)00523-1

Ostner, J., Kappeler, P., \& Heistermann, M. (2008). Androgen and glucocorticoid levels reflect seasonally occurring social challenges in male redfronted lemurs (Eulemur fulvus rufus). Behavioral Ecology and Sociobiology, 62(4), 627-638. https://doi.org/10.1007/s00265-007-0487$\mathrm{y}$

Ostner, J., \& Schülke, O. (2014). The evolution of social bonds in primate males. Behaviour, 151 (7), 871-906. https://doi.org/10.1163/1568539X-00003191

Ostner, J., \& Schülke, O. (2018). Linking sociality to fitness in primates: A call for mechanisms. In M. Naguib, L. Barrett, S. D. Healy, J. Podos, L. W. Simmons, \& M. Zuk (Eds.), Advances in the Study of Behavior (Vol. 50, pp. 127-175). Academic Press. https://doi.org/10.1016/bs. asb.2017.12.001

Oyegbile, T. O., \& Marler, C. A. (2006). Weak winner effect in a less aggressive mammal: Correlations with corticosterone but not testosterone. Physiology \& Behavior, 89(2), 171-179. https://doi.org/10.1016/j.physbeh.2006.05.044

Palme, R. (2019). Non-invasive measurement of glucocorticoids: Advances and problems. Physiology \& Behavior, 199, 229-243. https://doi.org/10.1016/j.physbeh.2018.11.021

Palme, R., \& Möstl, E. (1994). Biotin-streptavidin enzyme immunoassay for the determination of oestrogens and androgens in boar faeces. In S. Görög (Ed.), Advances in steroid analysis '93 (pp. 111-117). Akademiai Kiado, Budapest.

Palme, R., \& Möstl, E. (1997). Measurement of cortisol metabolites in faeces of sheep as a parameter of cortisol concentration in blood. International Journal of Mammalian Biology, 62, 192-197.

Paul, A., Kuester, J., \& Arnemann, J. (1996). The sociobiology of male-infant interactions in Barbary macaques, Macaca sylvanus. Animal Behaviour, 51(1), 155-170. https://doi.org/10. 1006/anbe.1996.0013

Paul, A., Preuschoft, S., \& van Schaik, C. P. (2000). The other side of the coin: Infanticide and the evolution of affiliative male-infant interactions in Old World primates. In C. P. van Schaik 
\& C. H. Janson (Eds.), Infanticide by Males and its Implications (pp. 269-292). Cambridge: Cambridge University Press. https://doi.org/10.1017/CBO9780511542312.014

Peciña, M., Love, T., Stohler, C. S., Goldman, D., \& Zubieta, J.-K. (2015). Effects of the mu opioid receptor polymorphism (OPRM1 A118G) on pain regulation, placebo effects and associated personality trait measures. Neuropsychopharmacology, 40(4), 957-965. https://doi. org/10.1038/npp.2014.272

Peters, M., Simmons, L. W., \& Rhodes, G. (2008). Testosterone is associated with mating success but not attractiveness or masculinity in human males. Animal Behaviour, 76(2), 297-303. https://doi.org/10.1016/j.anbehav.2008.02.008

Pineda-Galindo, E., Cerda-Molina, A. L., Mayagoitia-Novales, L., Matamoros-Trejo, G., \& de la O, C. (2017). Biological validations of fecal glucocorticoid, testosterone, and progesterone metabolite measurements in captive stumptail macaques (Macaca arctoides). International Journal of Primatology, 38(6), 985-1001. https://doi.org/10.1007/s10764-017-9992-7

Preis, A., Mugisha, L., Hauser, B., Weltring, A., \& Deschner, T. (2011). Androgen and androgen metabolite levels in serum and urine of East African chimpanzees (Pan troglodytes schweinfurthii): Comparison of EIA and LC-MS analyses. General and Comparative Endocrinology, 174 (3), 335-343. https://doi.org/10.1016/j.ygcen.2011.09.010

Preis, A., Samuni, L., Mielke, A., Deschner, T., Crockford, C., \& Wittig, R. M. (2018). Urinary oxytocin levels in relation to post-conflict affiliations in wild male chimpanzees (Pan troglodytes verus). Hormones and Behavior, 105, 28-40. https://doi.org/10.1016/j.yhbeh.2018.07.009

Preuschoft, S., \& Paul, A. (2000). Dominance, egalitarianism, and stalemate: An experimental approach to male-male competition in Barbary macaques. In Primate males: Causes and consequences of variation in group composition (pp. 205-216).

Raulo, A., \& Dantzer, B. (2018). Associations between glucocorticoids and sociality across a continuum of vertebrate social behavior. Ecology and Evolution, 0(0). https://doi.org/10.1002/ ece3.4059

R Core Team. (2018). R: A language and environment for statistical computing. Vienna, Austria: R Foundation for Statistical Computing.

Reyes, T. L., Galinsky, A. M., Hoffmann, J. N., You, H. M., Ziegler, T. E., \& McClintock, M. K. (2014). Social peptides: Measuring urinary oxytocin and vasopressin in a home field study of older adults at risk for dehydration. The Journals of Gerontology: Series B, 69(Suppl_2), S229-S237. https://doi.org/10.1093/geronb/gbu104

Rilling, J. K., \& Young, L. J. (2014). The biology of mammalian parenting and its effect on 
offspring social development. Science, 345(6198), 771-776. https://doi.org/10.1126/science. 1252723

Rincon, A. V., Deschner, T., Schülke, O., \& Ostner, J. (2019a). Oxytocin increases after affiliative interactions in male Barbary macaques. bioRxiv, 695064. https://doi.org/10.1101/ 695064

Rincon, A. V., Maréchal, L., Semple, S., Majolo, B., \& MacLarnon, A. (2017). Correlates of androgens in wild male Barbary macaques: Testing the challenge hypothesis. American Journal of Primatology, 79(10), e22689. https://doi.org/10.1002/ajp.22689

Rincon, A. V., Ostner, J., Heistermann, M., \& Deschner, T. (2019b). Measuring urinary cortisol and testosterone levels in male Barbary macaques: A comparison of EIA and LC-MS. General and Comparative Endocrinology, 281, 117-125. https://doi.org/10.1016/j.ygcen.2019.05.017

Robbins, M. M., \& Czekala, N. M. (1997). A preliminary investigation of urinary testosterone and cortisol levels in wild male mountain gorillas. American Journal of Primatology, 43(1), 51-64. https://doi.org/10.1002/(SICI)1098-2345(1997)43:1\%3C51::AID-AJP4\%3E3.0.CO;2-X

Robinson, I. C. a. F., \& Jones, P. M. (1982). Oxytocin and neurophysin in plasma and CSF during suckling in the guinea-pig. Neuroendocrinology, 34(1), 59-63. https://doi.org/10.1159/ 000123278

Romero, L. M., Dickens, M. J., \& Cyr, N. E. (2009). The reactive scope model - a new model integrating homeostasis, allostasis, and stress. Hormones and Behavior, 55(3), 375-389. https://doi.org/10.1016/j.yhbeh.2008.12.009

Romero, T., Nagasawa, M., Mogi, K., Hasegawa, T., \& Kikusui, T. (2014). Oxytocin promotes social bonding in dogs. Proceedings of the National Academy of Sciences, 111(25), 9085-9090. https://doi.org/10.1073/pnas.1322868111

Ronay, R., \& Carney, D. R. (2013). Testosterone's negative relationship with empathic accuracy and perceived leadership ability. Social Psychological and Personality Science, 4(1), 92-99. https://doi.org/10.1177/1948550612442395

Roney, J. R., \& Gettler, L. T. (2015). The role of testosterone in human romantic relationships. Current Opinion in Psychology, 1, 81-86. https://doi.org/10.1016/j.copsyc.2014.11.003

Rosal, M. C., King, J., Ma, Y., \& Reed, G. W. (2004). Stress, social support, and cortisol: Inverse associations? Behavioral Medicine, 30(1), 11-22. https://doi.org/10.3200/BMED.30.1. $11-22$

Ross, H. E., Cole, C. D., Smith, Y., Neumann, I. D., Landgraf, R., Murphy, A. Z., \& Young, L. J. 
(2009). Characterization of the oxytocin system regulating affiliative behavior in female prairie voles. Neuroscience, 162(4), 892-903. https://doi.org/10.1016/j.neuroscience.2009.05.055

Ross, H. E., \& Young, L. J. (2009). Oxytocin and the neural mechanisms regulating social cognition and affiliative behavior. Frontiers in Neuroendocrinology, 30(4), 534-547. https: //doi.org/10.1016/j.yfrne.2009.05.004

Ryder, T. B., Dakin, R., Vernasco, B. J., Evans, B. S., Horton, B. M., \& Moore, I. T. (2019). Testosterone modulates status-specific patterns of cooperation in a social network. bioRxiv, 453548. https://doi.org/10.1101/453548

Salvador, A. (2012). Steroid hormones and some evolutionary-relevant social interactions. Motivation and Emotion, 36(1), 74-83. https://doi.org/10.1007/s11031-011-9265-2

Samuni, L., Preis, A., Deschner, T., Crockford, C., \& Wittig, R. M. (2018a). Reward of labor coordination and hunting success in wild chimpanzees. Communications Biology, 1(1), 138. https://doi.org/10.1038/s42003-018-0142-3

Samuni, L., Preis, A., Mielke, A., Deschner, T., Wittig, R. M., \& Crockford, C. (2018b). Social bonds facilitate cooperative resource sharing in wild chimpanzees. Proceedings of the Royal Society B, 285(1888), 20181643. https://doi.org/10.1098/rspb.2018.1643

Samuni, L., Preis, A., Mundry, R., Deschner, T., Crockford, C., \& Wittig, R. M. (2017). Oxytocin reactivity during intergroup conflict in wild chimpanzees. Proceedings of the National Academy of Sciences, 114(2), 268-273. https://doi.org/10.1073/pnas.1616812114

Sapolsky, R. M. (2002). Endocrinology of the stress-response. In J. B. Becker, S. M. Breedlove, D. Crews, \& M. M. McCarthy (Eds.), Behavioral Endocrinology (2nd ed., pp. 409-450). Cambridge, MA: MIT Press.

Schielzeth, H., \& Forstmeier, W. (2009). Conclusions beyond support: Overconfident estimates in mixed models. Behavioral Ecology, 20(2), 416-420. https://doi.org/10.1093/beheco/arn145

Schino, G., \& Aureli, F. (2009). Reciprocal altruism in primates: Partner choice, cognition, and emotions. In Advances in the Study of Behavior (Vol. 39, pp. 45-69). Academic Press. https://doi.org/10.1016/S0065-3454(09)39002-6

Schmid, J., Heistermann, M., Ganslosser, U., \& Hodges, J. K. (2001). Introduction of foreign female Asian elephants (Elephas maximus) into an existing group: Behavioural reactions and changes in cortisol levels. Animal Welfare, 10(4), 357-372.

Schülke, O., Bhagavatula, J., Vigilant, L., \& Ostner, J. (2010). Social bonds enhance reproductive success in male macaques. Current Biology, 20(24), 2207-2210. https: 
//doi.org/10.1016/j.cub.2010.10.058

Seltzer, L. J., \& Ziegler, T. E. (2007). Non-invasive measurement of small peptides in the common marmoset (Callithrix jacchus): A radiolabeled clearance study and endogenous excretion under varying social conditions. Hormones and Behavior, 51(3), 436-442. https://doi.org/10.1016/j.yhbeh.2006.12.012

Setchell, J. M., Smith, T., Wickings, E. J., \& Knapp, L. A. (2008). Social correlates of testosterone and ornamentation in male mandrills. Hormones and Behavior, 54 (3), 365-372. https://doi.org/10.1016/j.yhbeh.2008.05.004

Setchell, J. M., Smith, T., Wickings, E. J., \& Knapp, L. A. (2010). Stress, social behaviour, and secondary sexual traits in a male primate. Hormones and Behavior, 58(5), 720-728. https: //doi.org/10.1016/j.yhbeh.2010.07.004

Seyfarth, R. M., \& Cheney, D. L. (2012). The evolutionary origins of friendship. Annual Review of Psychology, 63(1), 153-177. https://doi.org/10.1146/annurev-psych-120710-100337

Shamay-Tsoory, S. G., \& Abu-Akel, A. (2016). The social salience hypothesis of oxytocin. Biological Psychiatry, 79(3), 194-202. https://doi.org/10.1016/j.biopsych.2015.07.020

Shutt, K., Setchell, J. M., \& Heistermann, M. (2012). Non-invasive monitoring of physiological stress in the Western lowland gorilla (Gorilla gorilla gorilla): Validation of a fecal glucocorticoid assay and methods for practical application in the field. General and Comparative Endocrinology, 179(2), 167-177. https://doi.org/10.1016/j.ygcen.2012.08.008

Silk, J. (2002). Using the 'F'-word in primatology. Behaviour, 139(2), 421-446. https://doi. org/10.1163/156853902760102735

Silk, J. B. (2007). The adaptive value of sociality in mammalian groups. Philosophical Transactions of the Royal Society B: Biological Sciences, 362(1480), 539-559. https: //doi.org/10.1098/rstb.2006.1994

Silk, J. B., Beehner, J. C., Bergman, T. J., Crockford, C., Engh, A. L., Moscovice, L. R., Wittig, R. M., Seyfarth, R. M., \& Cheney, D. L. (2009). The benefits of social capital: Close social bonds among female baboons enhance offspring survival. Proceedings of the Royal Society B, 276(1670), 3099-3104. https://doi.org/10.1098/rspb.2009.0681

Silk, J. B., Beehner, J. C., Bergman, T. J., Crockford, C., Engh, A. L., Moscovice, L. R., Wittig, R. M., Seyfarth, R. M., \& Cheney, D. L. (2010). Strong and consistent social bonds enhance the longevity of female baboons. Current Biology, 20(15), 1359-1361. https://doi.org/10.1016/ j.cub.2010.05.067 
Skuse, D. H., \& Gallagher, L. (2009). Dopaminergic-neuropeptide interactions in the social brain. Trends in Cognitive Sciences, 13(1), 27-35. https://doi.org/10.1016/j.tics.2008.09.007

Slatcher, R. B., Mehta, P. H., \& Josephs, R. A. (2011). Testosterone and self-reported dominance interact to influence human mating behavior. Social Psychological and Personality Science, 2(5), 531-539. https://doi.org/10.1177/1948550611400099

Small, M. F. (1990). Promiscuity in Barbary macaques (Macaca sylvanus). American Journal of Primatology, 20(4), 267-282. https://doi.org/10.1002/ajp.1350200403

Smith, A. S., Ågmo, A., Birnie, A. K., \& French, J. A. (2010). Hormones and Behavior, 57(2), 255-262. https://doi.org/10.1016/j.yhbeh.2009.12.004

Smith, A. S., \& Wang, Z. (2012). Salubrious effects of oxytocin on social stress-induced deficits. Hormones and Behavior, 61(3), 320-330. https://doi.org/10.1016/j.yhbeh.2011.11.010

Smith, A. S., \& Wang, Z. (2014). Hypothalamic oxytocin mediates social buffering of the stress response. Biological Psychiatry, 76 (4), 281-288. https://doi.org/10.1016/j.biopsych.2013.09. 017

Smith, J. E., Powning, K. S., Dawes, S. E., Estrada, J. R., Hopper, A. L., Piotrowski, S. L., \& Holekamp, K. E. (2011). Greetings promote cooperation and reinforce social bonds among spotted hyaenas. Animal Behaviour, 81(2), 401-415. https://doi.org/10.1016/j.anbehav.2010. 11.007

Snowdon, C. T., Pieper, B. A., Boe, C. Y., Cronin, K. A., Kurian, A. V., \& Ziegler, T. E. (2010). Variation in oxytocin is related to variation in affiliative behavior in monogamous, pairbonded tamarins. Hormones and Behavior, 58(4), 614-618. https://doi.org/10.1016/j.yhbeh.2010.06. 014

Soares, M. C., Bshary, R., Fusani, L., Goymann, W., Hau, M., Hirschenhauser, K., \& Oliveira, R. F. (2010). Hormonal mechanisms of cooperative behaviour. Philosophical Transactions of the Royal Society B: Biological Sciences, 365(1553), 2737-2750. https://doi.org/10.1098/rstb. 2010.0151

Sobolewski, M. E., Brown, J. L., \& Mitani, J. C. (2012). Territoriality, tolerance and testosterone in wild chimpanzees. Animal Behaviour, 84(6), 1469-1474. https://doi.org/10.1016/j.anbehav. 2012.09.018

Sobolewski, M. E., Brown, J. L., \& Mitani, J. C. (2013). Female parity, male aggression, and the challenge hypothesis in wild chimpanzees. Primates, 54(1), 81-88. https://doi.org/10.1007/ s10329-012-0332-4 
Sonnweber, R. S., Ravignani, A., Stobbe, N., Schiestl, G., Wallner, B., \& Fitch, W. T. (2015). Rank-dependent grooming patterns and cortisol alleviation in Barbary macaques. American Journal of Primatology, r7y(6), 688-700. https://doi.org/10.1002/ajp.22391

Stanton, M. A., \& Mann, J. (2012). Early social networks predict survival in wild bottlenose dolphins. PLOS ONE, 7(10), e47508. https://doi.org/10.1371/journal.pone.0047508

Stevenson, E. T., Gese, E. M., Neuman-Lee, L. A., \& French, S. S. (2018). Levels of plasma and fecal glucocorticoid metabolites following an ACTH challenge in male and female coyotes (Canis latrans). Journal of Comparative Physiology B, 188(2), 345-358. https://doi.org/10. $1007 / \mathrm{s} 00360-017-1125-2$

Stiver, K. A., \& Alonzo, S. H. (2009). Parental and mating effort: Is there necessarily a trade-off? Ethology, 115(12), 1101-1126. https://doi.org/10.1111/j.1439-0310.2009.01707.x

Stocker, M., Munteanu, A., Stöwe, M., Schwab, C., Palme, R., \& Bugnyar, T. (2016). Loner or socializer? Ravens' adrenocortical response to individual separation depends on social integration. Hormones and Behavior, 78, 194-199. https://doi.org/10.1016/j.yhbeh.2015.11.009

Strauss, E. D., \& Holekamp, K. E. (2019). Social alliances improve rank and fitness in convention-based societies. Proceedings of the National Academy of Sciences, 201810384. https://doi.org/10.1073/pnas.1810384116

Tarr, B., Launay, J., Cohen, \& Dunbar, R. I. M. (2015). Synchrony and exertion during dance independently raise pain threshold and encourage social bonding. Biology Letters, 11(10), 20150767. https://doi.org/10.1098/rsbl.2015.0767

Tarr, B., Launay, J., \& Dunbar, R. I. M. (2016). Silent disco: Dancing in synchrony leads to elevated pain thresholds and social closeness. Evolution and Human Behavior, 37(5), 343-349. https://doi.org/10.1016/j.evolhumbehav.2016.02.004

Taylor, W. (1971). The excretion of steroid hormone metabolites in bile and feces. In R. S. Harris, E. Diczfalusy, P. L. Munson, J. Glover, K. V. Thimann, I. G. Wool, \& J. A. Loraine (Eds.), Vitamins $\&$ Hormones (Vol. 29, pp. 201-285). Academic Press. https://doi.org/10. 1016/S0083-6729(08)60050-3

Ten Velden, F. S., Daughters, K., \& De Dreu, C. K. W. (2017). Oxytocin promotes intuitive rather than deliberated cooperation with the in-group. Hormones and Behavior, 92, 164-171. https://doi.org/10.1016/j.yhbeh.2016.06.005

Terburg, D., Morgan, B., \& van Honk, J. (2009). The testosterone-cortisol ratio: A hormonal marker for proneness to social aggression. International Journal of Law and Psychiatry, 32(4), 216-223. https://doi.org/10.1016/j.ijlp.2009.04.008 
Teskey-Gerstl, A., Bamberg, E., Steineck, T., \& Palme, R. (2000). Excretion of corticosteroids in urine and faeces of hares (Lepus europaeus). Journal of Comparative Physiology B, 170(2), 163-168. https://doi.org/10.1007/s003600050271

Thierry, B., Singh, M., \& Kaumanns, W. (2004). Macaque societies: A model for the study of social organization. Cambridge University Press.

Torner, L., Plotsky, P. M., Neumann, I. D., \& de Jong, T. R. (2017). Forced swimming-induced oxytocin release into blood and brain: Effects of adrenalectomy and corticosterone treatment. Psychoneuroendocrinology, 77, 165-174. https://doi.org/10.1016/j.psyneuen.2016.12.006

Touma, C., \& Palme, R. (2005). Measuring fecal glucocorticoid metabolites in mammals and birds: The importance of validation. Annals of the New York Academy of Sciences, 1046(1), 54-74. https://doi.org/10.1196/annals.1343.006

Touma, C., Sachser, N., Möstl, E., \& Palme, R. (2003). Effects of sex and time of day on metabolism and excretion of corticosterone in urine and feces of mice. General and Comparative Endocrinology, 130(3), 267-278. https://doi.org/10.1016/S0016-6480(02)00620-2

Trivers, R. L. (1971). The evolution of reciprocal altruism. The Quarterly Review of Biology, 46(1), 35-57. https://doi.org/10.1086/406755

van Anders, S. M. (2013). Beyond masculinity: Testosterone, gender/sex, and human social behavior in a comparative context. Frontiers in Neuroendocrinology, 34(3), 198-210. https: //doi.org/10.1016/j.yfrne.2013.07.001

van Anders, S. M., \& Goldey, K. L. (2010). Testosterone and partnering are linked via relationship status for women and "relationship orientation" for men. Hormones and Behavior, 58(5), 820-826. https://doi.org/10.1016/j.yhbeh.2010.08.005

van Anders, S. M., Goldey, K. L., \& Kuo, P. X. (2011). The steroid/peptide theory of social bonds: Integrating testosterone and peptide responses for classifying social behavioral contexts. Psychoneuroendocrinology, 36(9), 1265-1275. https://doi.org/10.1016/j.psyneuen.2011.06.001

van Anders, S. M., Tolman, R. M., \& Jainagaraj, G. (2014). Examining how infant interactions influence men's hormones, affect, and aggression using the Michigan infant nurturance simulation paradigm. Fathering, 12(2), 143. https://doi.org/DOI:10.3149/fth.1202.143

van Anders, S. M., Tolman, R. M., \& Volling, B. L. (2012). Baby cries and nurturance affect testosterone in men. Hormones and Behavior, 61(1), 31-36. https://doi.org/10.1016/j.yhbeh. 2011.09.012

van Honk, J., Schutter, D. J., Bos, P. A., Kruijt, A.-W., Lentjes, E. G., \& Baron-Cohen, 
S. (2011). Testosterone administration impairs cognitive empathy in women depending on second-to-fourth digit ratio. Proceedings of the National Academy of Sciences, 108(8), 3448-3452. https://doi.org/10.1073/pnas.1011891108

Venturelli, E., Cavalleri, A., \& Secreto, G. (1995). Methods for urinary testosterone analysis. Journal of Chromatography B: Biomedical Sciences and Applications, 671(1), 363-380. https: //doi.org/10.1016/0378-4347(95)00062-N

Viau, V. (2002). Functional cross-talk between the hypothalamic-pituitary-gonadal and -adrenal axes. Journal of Neuroendocrinology, 14(6), 506-513. https://doi.org/10.1046/j.1365-2826. 2002.00798.x

Wasser, S. K., Hunt, K. E., Brown, J. L., Cooper, K., Crockett, C. M., Bechert, U., Millspaugh, J. J., Larson, S., \& Monfort, S. L. (2000). A generalized fecal glucocorticoid assay for use in a diverse array of nondomestic mammalian and avian species. General and Comparative Endocrinology, 120(3), 260-275. https://doi.org/10.1006/gcen.2000.7557

Wasser, S. K., Risler, L., \& Steiner, R. A. (1988). Excreted steroids in primate feces over the menstrual cycle and pregnancy. Biology of Reproduction, 39(4), 862-872. https://doi.org/10. 1095/biolreprod39.4.862

Watts, D. P. (2002). Reciprocity and interchange in the social relationships of wild male chimpanzees. Behaviour, $139(2 / 3), 343-370$.

Weidt, A., Hofmann, S. E., \& König, B. (2008). Not only mate choice matters: Fitness consequences of social partner choice in female house mice. Animal Behaviour, 75 (3), 801-808. https://doi.org/10.1016/j.anbehav.2007.06.017

Welker, K. M., Lassetter, B., Brandes, C. M., Prasad, S., Koop, D. R., \& Mehta, P. H. (2016). A comparison of salivary testosterone measurement using immunoassays and tandem mass spectrometry. Psychoneuroendocrinology, 71, 180-188. https://doi.org/10.1016/j.psyneuen. 2016.05 .022

Welling, L. L. M., Jones, B. C., DeBruine, L. M., Smith, F. G., Feinberg, D. R., Little, A. C., \& Al-Dujaili, E. A. S. (2008). Men report stronger attraction to femininity in women's faces when their testosterone levels are high. Hormones and Behavior, 54(5), 703-708. https: //doi.org/10.1016/j.yhbeh.2008.07.012

Wessling, E. G., Kühl, H. S., Mundry, R., Deschner, T., \& Pruetz, J. D. (2018). The costs of living at the edge: Seasonal stress in wild savanna-dwelling chimpanzees. Journal of Human Evolution, 121, 1-11. https://doi.org/10.1016/j.jhevol.2018.03.001

Whitten, P. L. (1987). Infants and adult males. In B. B. Smuts (Ed.), Primate societies (pp. 
343-357). Chicago: University of Chicago Press.

Wingfield, J. C., Goymann, W., Jalabert, C., \& Soma, K. K. (2019). Concepts derived from the challenge hypothesis. Hormones and Behavior, 115, 104550. https://doi.org/10.1016/j.yhbeh. 2019.06 .014

Wingfield, J. C., Hegner, R. E., Dufty, A. M., \& Ball, G. F. (1990). The "challenge hypothesis": Theoretical implications for patterns of testosterone secretion, mating systems, and breeding strategies. The American Naturalist, 136(6), 829-846. https://doi.org/10.1086/285134

Wittig, R. M., Crockford, C., Deschner, T., Langergraber, K. E., Ziegler, T. E., \& Zuberbühler, K. (2014). Food sharing is linked to urinary oxytocin levels and bonding in related and unrelated wild chimpanzees. Proceedings of the Royal Society B, 281 (1778), 20133096. https://doi.org/ 10.1098/rspb.2013.3096

Wittig, R. M., Crockford, C., Weltring, A., Langergraber, K. E., Deschner, T., \& Zuberbühler, K. (2016). Social support reduces stress hormone levels in wild chimpanzees across stressful events and everyday affiliations. Nature Communications, 7, 13361. https://doi.org/10.1038/ ncomms13361

Wotjak, C. T., Ganster, J., Kohl, G., Holsboer, F., Landgraf, R., \& Engelmann, M. (1998). Dissociated central and peripheral release of vasopressin, but not oxytocin, in response to repeated swim stress: New insights into the secretory capacities of peptidergic neurons. Neuroscience, 85 (4), 1209-1222. https://doi.org/10.1016/S0306-4522(97)00683-0

Young, C., Hähndel, S., Majolo, B., Schülke, O., \& Ostner, J. (2013a). Male coalitions and female behaviour affect male mating success independent of dominance rank and female receptive synchrony in wild Barbary macaques. Behavioral Ecology and Sociobiology, 67(10), 1665-1677. https://doi.org/10.1007/s00265-013-1577-7

Young, C., Majolo, B., Heistermann, M., Schülke, O., \& Ostner, J. (2013b). Male mating behaviour in relation to female sexual swellings, socio-sexual behaviour and hormonal changes in wild Barbary macaques. Hormones and Behavior, 63(1), 32-39. https://doi.org/10.1016/j. yhbeh.2012.11.004

Young, C., Majolo, B., Heistermann, M., Schülke, O., \& Ostner, J. (2014a). Responses to social and environmental stress are attenuated by strong male bonds in wild macaques. Proceedings of the National Academy of Sciences, 111(51), 18195-18200. https://doi.org/10.1073/pnas. 1411450111

Young, C., Majolo, B., Schülke, O., \& Ostner, J. (2014b). Male social bonds and rank predict supporter selection in cooperative aggression in wild Barbary macaques. Animal Behaviour, 95, 23-32. https://doi.org/10.1016/j.anbehav.2014.06.007 
Young, C., Schülke, O., \& Ostner, J. (2014c). How males form coalitions against group rivals and the Pandit/van Schaik coalition model. Behaviour, 151(7), 907-934. https://doi.org/10. 1163/1568539X-00003166

Young, L. J., \& Wang, Z. (2004). The neurobiology of pair bonding. Nature Neuroscience, 7(10), 1048-1054. https://doi.org/10.1038/nn1327

Zhou, W.-X., Sornette, D., Hill, R. A., \& Dunbar, R. I. M. (2005). Discrete hierarchical organization of social group sizes. Proceedings of the Royal Society B, 272(1561), 439-444. https://doi.org/10.1098/rspb.2004.2970

Ziegler, T. E. (2018). Measuring peripheral oxytocin and vasopressin in nonhuman primates. American Journal of Primatology, 0(0), e22871. https://doi.org/10.1002/ajp.22871

Ziegler, T. E., Carlson, A. A., Ginther, A. J., \& Snowdon, C. T. (2000). Gonadal source of testosterone metabolites in urine of male cotton-top tamarin monkeys (Saguinus oedipus). General and Comparative Endocrinology, 118(2), 332-343. https://doi.org/10.1006/gcen.2000. 7476

Ziegler, T. E., \& Crockford, C. (2017). Neuroendocrine control in social relationships in non-human primates: Field based evidence. Hormones and Behavior, 91, 107-121. https: //doi.org/10.1016/j.yhbeh.2017.03.004 


\section{Acknowledgements}

The benefits of having strong social bonds become apparent when one embarks on a long journey. The completion of this thesis would not have been possible without considerable support from a number of people.

I have been fortunate to have some great mentors throughout this time. Thank you to Prof. Dr. Julia Ostner and Dr. Oliver Schülke for giving me the opportunity to undertake such a cool and exciting project. Thank you for always being available for discussions, keeping me on the right track, and making sure to keep looking at the bigger picture.

Thanks to Dr. Tobias Deschner and Dr. Michael Heistermann for continued support during my many months in the lab, masterminding the validation study in this thesis, and patiently answering what I'm sure were some very naïve questions. Also thank you to all lab technicians, especially to Vera Schmeling for showing me how to conduct a hormone deconjugation and extraction, and Andrea Heistermann for conducting the analysis of urinary cortisol as well as general assistance in the lab.

Thank you to Roland Hilgartner and Ellen Merz for permission to conduct the study at Affenberg Salem. Special thanks also to Mamisolo Hilgartner for introducing me to the monkeys and having someone to keenly chat about monkey gossip. Successful completion of data would not have been possible without some fantastic field assistants. Thank you Tatjana Kaufmann, Lilah Sciacky, and Lauren Cassidy for collecting great data, providing good company, and maintaining a great atmosphere despite some long working days. Field work is much more enjoyable with great friends.

Thanks you to Prof. Dr. Julia Fischer and the other PIs of the RTG 2070 "Understanding Social Relationships", for providing such a great environment for scientific discussions with PIs and students of different research areas, and making it possible (affordable) to visit other departments and attend scientific conferences. Thanks to our fantastic coordinator Dr. Rebecca Jürgens, who made many technical aspects of conducting a $\mathrm{PhD}$ infinitely easier and freeing up time for the things that count.

Thanks of course to all my department collogues and friends for such great company through the years. Thanks to Delphine de Moor for ensuring talks about the meaning of life were never far away and for showing me that it is possible not to age at all during a PhD. Thanks to Dr. Nadine Müller-Klein for making sure I didn't work too long without socializing in the office and was never left wanting for cake or cat videos. Special thanks again to Delphine and Nadine for making such a good conferencing team. Thanks to Dr. Federica Dal Pesco for being my unofficial statistician and such a cool friend. Not to be underestimated, thanks to Matthis Drolet for ensuring that not all social gatherings were exclusively talk about primate behavior, but that one can also be a nerd about technology, science fiction, and the foundational laws of 
the universe.

Last but not least, I am forever grateful to Lauren Cassidy who has been by my side throughout this journey. From collecting behavioral data and (literally) hundreds of urine samples, to continued feedback and discussions on early versions of manuscripts including this thesis, and most of all for the enduring emotional support that makes the journey more enjoyable than the destination. 


\section{Declaration}

I hereby declare that I have written this thesis entitled "The role of oxytocin, testosterone and cortisol in affiliation and bonding in male Barbary macaques" independently and with no other aids or sources than quoted.

Alan V. Rincon

Göttingen, 2019 